
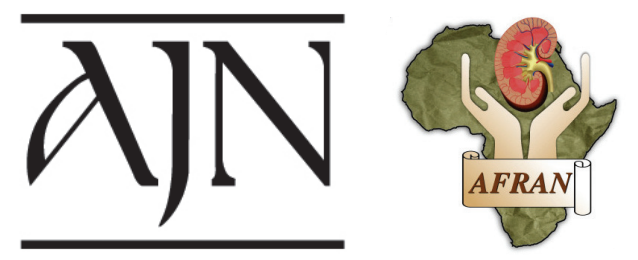

\title{
CONGRESS PROCEEDINGS
}

Abstracts of the joint AFRAN and AFPNA Congress, hosted by the Kenya Renal Association, held in Mombasa, Kenya, 25-28 September 2019 


\title{
ACUTE KIDNEY INJURY
}

\section{Abstract 1: \\ EVOLUTION OF ACUTE KIDNEY INJURY IN DENGUE FEVER IN A NEPHROLOGY DEPARTMENT IN OUAGADOUGOU (BURKINA FASO)}

\author{
Habin'Yabama Aida LENGANI ${ }^{1}$, Delwendé Bertrand DIBRI ${ }^{1}$, Gaoussou SANOU ${ }^{1}$, Joel Ignace NITIÉMA ${ }^{1}$, Gérard \\ COULIBALY' \\ ${ }^{1}$ Department of Nephrology, University Hospital Yalgado Ouédraogo, Ouagadougou, Burkina Faso.
}

Objectives: The aim of this study was to describe the clinical, biological and evolutionary profile of acute kidney injury (AKI) in dengue fever.

Methods: This was a prospective study conducted from October 1, 2016 to March 31, 2017 in the Department of Nephrology and Hemodialysis of the University Hospital Yalgado Ouédraogo. All patients hospitalized for AKI during dengue fever and followed for at least 3 months after hospital discharge were included. AKI was defined and classified into 3 stages according to the KDIGO criteria.

Results: Thirty patients, with an average age of $37.23 \pm 15.80$ years, were followed. The sex ratio was 3.28 . Fever, vomiting, hemorrhagic syndrome and oligo-anuria were present in $100 \%, 53.33 \%, 43.33 \%$ and $33.33 \%$ of patients respectively. The mean creatinine level was $835.91 \pm 771.52 \mu \mathrm{mol} / \mathrm{L}$. Regarding the severity of AKI, $73.33 \%$ of patients were classified in KDIGO stage 3. Metabolic acidosis, hyponatremia and hyperkalemia were present in $76.67 \%, 56.67 \%$ and $16.67 \%$ of patients, respectively. Nine patients (30\%) received hemodialysis therapy for acute lung edema in 5 patients. The average length of hospitalization was $13.27 \pm 6.51$ days. At discharge, recovery of renal function was complete in 25 patients (83.33\%), partial in 4 patients (13.33\%) and one patient (3.33\%) deceased. At the third month of follow-up, $90 \%$ of patients had normal renal function with an average creatinine level of $93.15 \pm 18.89 \mu \mathrm{mol} / \mathrm{L}$. One patient (3.33\%) had persistent renal failure and one patient (3.33\%) deceased.

Conclusions: The evolution of AKI in dengue fever is favorable when care is provided early and in a specialized medical unit.

\section{Abstract 2: \\ FACTORS ASSOCIATED WITH THE ACUTE RENAL FAILURE OCCURRENCE DURING DENGUE FEVER ASSOCIATED IN OUAGADOUGOU (BURKINA FASO)}

\author{
J.Y. BONZI ${ }^{1}$, A. LENGANI', A. SONDO ${ }^{1}$, A. ZOUNDI ${ }^{2}$, A. PODA ${ }^{3}$, I. J. NITIEMA ${ }^{1}$, G. SANOU ${ }^{1}$, H. TRAORE ${ }^{1}$, G. COULIBALY ${ }^{1}$ \\ 1-Nephrology-Hemodialysis Departement of Yalgado Ouédraogo University Hospital, 2-University of Joseph KI-zerbo, \\ sOuagadougou, 3-Infectious Disease Departement of Sanou Souro University Hospital.
}

Objective: Dengue fever is a common arbovirus in tropical countries; its progression can be complicated by ARF due to death, admission to intensive care and prolonged hospitalization. We report here on a study on factors associated with ARF during dengue fever in Ouagadougou.

Methods: This was a 15-month cross-sectional study conducted in 15 hospitals in Ouagadougou. All cases of dengue fever were reported and patients over 16 years of age with creatinine $\geq 176.5 \mu \mathrm{mol} / \mathrm{L}$ were included. Patients with pre-existing renal disease were not included. Clinical and paraclinical data were noted. ARF was defined as creatinine $>176.5 \mu \mathrm{mol} / \mathrm{L}$ in a patient with normal prior renal function.

Results: We recorded 816 cases of dengue fever, creatinine levels were found in 487 cases (59.7\%) and of these 430 cases were over 16 years of age, 53 of which had creatinine levels of $176.5 \mu \mathrm{mol} / \mathrm{L}$. The average age was $42 \pm 17.3$ years and the 15 - to 30 -year-old age group was the most represented in $60.3 \%$ of cases. The main signs/syndromes were fever (83\%), arthralgia and myalgia (62.6\%), haemorrhagic syndrome $(17 \%)$ and clinical anemia. Biologically, the mean creatinine level was $1000 \pm 840 \mu \mathrm{mol} / \mathrm{L}$ and the mean hemoglobin level was $10.37 \mathrm{~g} / \mathrm{dL}$. Hepatic cytolysis and anemia were present in 26 cases (65\%) and 34 cases $(66.7 \%)$ respectively. Malaria-dengue co-infection was present in 13 cases. Factors associated with ARF were age over 60 years, self-medication, high blood pressure, hemorrhagic syndrome, anemia, hepatic cytolysis and prothrombin levels below 70\%. Eight cases (15.09\%) were taken for hemodialysis at least once on the basis of severity criteria and thirteen patients $(24.52 \%)$ died.

Conclusions: ARF is common during dengue fever. It is multifactorial and can affect patients' vital prognosis, especially in our context of limited resources. 


\section{Abstract 3:}

\section{AETIOLOGIES AND PROGNOSIS OF ACUTE RENAL FAILURE IN HEMODIALYSIS IN BURUNDI}

Nyandwi $\mathrm{J}^{1}$, Ndirahisha $\mathrm{E}^{2}$, Manirakiza $\mathrm{S}^{3}$, Harakandi $\mathrm{S}^{4}$, Niyonkuru $\mathrm{F}^{1}$

${ }^{1}$ Unité d'hémodialyse, Centre hospitalo-universitaire de Kamenge, ${ }^{2}$ Unité de Cardiologie, Centre hospitalouniversitaire de Kamenge, ${ }^{3}$ Département d'imagerie médicale, Centre hospitalo-universitaire de Kamenge, ${ }^{4}$ Département d'anesthésié-réanimation, Centre hospitalo-universitaire de Kamenge.

Introduction: Acute renal failure is a particularly common complication in the intensive care setting, and often requires the use of renal replacement therapy in emergency.

Objective: To determine the etiology and prognosis of acute renal failure in hemodialysis in Burundi.

Methods: This is a retrospective study of 46 emergency dialysis patients in 3 dialysis units in Burundi (Burundi Kidney Care clinic, Prince Regent Charles Hospital and Kamenge University Hospital Center) between July 2014 and March 2018.

Results: The mean age of the patients was $36.7 \pm 16$ years with extremes of 4 and 71 years. The male sex predominated (67.4\%). Acute renal failure was medical (91.3\%), surgical (4.3\%) and gynecological-obstetrical (4.3\%). The etiologies were severe malaria (73.9\%), acute non-febrile gastroenteritis (6.5\%), post-rhabdomyolysis (6.5\%), acute drug poisoning (4.3\%), externalized hemorrhage $(4.3 \%)$ and alcohol intoxication (2.2\%). Indications for hemodialysis were acute pulmonary edema (28.3\%), uremic encephalopathy (23.9\%), and anuria over 24 hours (21.7\%), threatening hyperkalemia (19.6\%), intoxication with dialyzable products (6.5\%). The dialysis was done on a femoral catheter in all patients. The average number of hemodialysis sessions to cover renal function was $3.30 \pm 1.71$ sessions. As outcomes we noted a mortality of $17.4 \%$, the normalization of the creatinine in $78.3 \%$ of the cases, and the evolution towards chronicity in 2 patients $(4,3 \%)$.

Conclusions: The main cause of acute renal failure in Burundi is malaria. Emergency hemodialysis contributes to the improvement of its prognosis even if the mortality remains high. The prevention of malaria is the basis to avoid acute renal failure in Burundi.

\section{Abstract 4: \\ RECOGNITION AND OUTCOME OF ACUTE KIDNEY INJURY IN ADULT NON-RENAL MEDICAL AND SURGICAL ADMISSIONS IN A TERTIARY HOSPITAL IN CAMEROON}

\section{Denis Georges Teuwafeu, Ayonghe Arisha Bisi, Gloria Ashuntantang}

Background: There is a paucity of data on the burden of AKI in non-renal medical and surgical admissions where renal function monitoring is not routine.

Objective: To study the incidence and outcomes of AKI in non-renal medical and surgical admissions at risk of AKI. Methods: We prospectively assessed non-renal medical and surgical admissions for AKI risk factors. We excluded patients with a history of CKD, confounders of serum creatinine $(\mathrm{SCr})$ and those without a second $\mathrm{SCr}$ value. Patients were followed up until hospital discharge or death. The outcome measures were presence of AKI, need and access to dialysis, renal recovery on discharge, for both participants with and without AKI, death and length of hospital stay. Results: A total of 165 (41.6\% males) participants were included. Their mean age was 50.7(17.29) years. Hypertension $(26.06 \%)$, obesity (16.97\%), HIV (15.15\%) and Diabetes Mellitus (13.33\%) were the most common comorbidities. Sepsis (66.67\%) and volume depletion (41.82\%) were the most frequent AKI risk factors. The incidence of AKI was $27.3 \%$ with $35.6 \%$ of these in stage 3 . A total of $4(8.9 \%)$ required dialysis with a $100 \%$ access rate. Mortality was significantly higher in the AKI group ( $17.8 \%$ vs $2.5 \%, p=0.001)$, and length of hospital stay was comparable in the AKI and non-AKI groups (11days vs 8 days, $p=0.103$ ). Renal recovery on discharge was complete in $62.2 \%$ of survivors. Conclusions: The incidence of AKI is high in non-renal medical and surgical admissions and it is associated with a high mortality. Early recognition through risk stratification and renal function monitoring may improve outcomes in AKI. 


\title{
Abstract 5:
}

\section{THE CLINICAL PATTERN AND OUTCOMES OF ACUTE KIDNEY INJURY IN A SEMI-URBAN HOSPITAL SETTING IN CAMEROON: THE CASE OF THE BUEA REGIONAL HOSPITAL.}

\author{
Shadrach Fritz KOMBE \\ Mbingo Baptist Hospital, Cameroon.
}

Background: Acute kidney injury (AKI) is a common disorder which is associated with high morbidity and mortality especially in the developing countries. In contrast to high income countries, AKI in these areas is mainly communityacquired and tends to affect the young. Previous studies on the epidemiology of AKI in Cameroon were carried out in urban tertiary hospitals, thus data on the clinical pattern and outcome in semi-urban setting are lacking and may be quite different.

Objective: To describe the clinical pattern and outcomes of AKI in Buea Regional Hospital.

Methods: We conducted a hospital-based observational retrospective study at the Buea Regional Hospital, a semiurban second category health facility in Cameroon. We excluded patients with known chronic kidney disease, and incomplete data. AKI was diagnosed and classified based on KDIGO criteria 2012 and renal recovery evaluated at 1 month.

Results: Of the 196 participants included, $57.7 \%$ were males and $10.7 \%$ children. The median age was 45 years. HIV infection, hypertension and diabetes were the most frequent comorbidities. AKI was community-acquired in $94.9 \%$ and $59.2 \%$ of participants had stage 3 AKI. Sepsis $37.2 \%$, volume depletion $24 \%$ and nephrotoxicity from herbal remedies $15.3 \%$ were the main causes of AKI. Post-abortum sepsis and malaria-related AKI accounted for most obstetric and pediatric AKI respectively. AKI was mainly renal $72 \%$ in mechanism with ATN accounting for $56.6 \%$. In all 71(36.2\%) participants had indications for dialysis but only 52(73.2\%) accessed it. Lack of appropriate dialysis technique, funds and stock-out of dialysis supplies were the main reasons for non-access. In-hospital mortality was $37.2 \%$. Among survivors, renal recovery was complete in $72.9 \%$, partial in $23.7 \%$, and no recovery in $3.4 \%$. Stage 3 disease was the only predictor of poor renal recovery at one month.

Conclusions: AKI in this semi-urban hospital setting is essentially community-acquired affecting the young and caused by infections, volume depletion and herbal toxins. Patients present late with severe disease, and about a third of them require dialysis.

\section{Abstract 6:}

\section{CLINICAL, PARACLINICAL AND OUTCOMES OF ACUTE KIDNEY INJURY ASSOCIATED WITH HEMOLYTIC ANEMIA IN YAOUNDÉ}

Cédric Xavier Mbobnda, Ronald Gobina, Linda NjonKam, Martial Tsiazok, Cécile Ngo Makang, Sylvia Santerre, Larissa Mbomena, François Kaze, Gloria Ashuntantang

Department of Internal Medicine and specialties of Faculty of Medicine and Biomedical Sciences, University of Yaoundé 1, Cameroon.

Objective: Describe the clinical and paraclinical features of acute kidney injury (AKI) associated with hemolytic anemia in Yaoundé and identify factors affecting the outcomes.

Methods: We carried out a retrospective study including 56 case files of adult patients hospitalized between January 2016 and January 2019 for AKI associated with hemolytic anemia at the Yaoundé General Hospital (YGH) and Yaoundé University Teaching Hospital (YUTH). Sociodemographic, clinical, paraclinical and outcomes parameter were recorded. Results: The mean age was $34.66 \pm 16.54$ years, with $33(58.9 \%)$ males. Fever (64.3\%), jaundice (60.7\%) and coca-cola urine $(51.8 \%)$ were the most frequent symptoms. Hypertension $(17.9 \%)$ and diabetes $(5.4 \%)$ were the main comorbidities. Toxic acute tubular necrosis (69.6\%) and ischemic acute tubular necrosis (39.3\%) were the most prevalent mechanism of injury. Kidney Injury occurred in the context of malaria (71.4\%), Sepsis (48.2\%) and thrombotic microangiopathy (32.1\%). Hemodialysis was performed in 36 (64.3\%) cases indicated for anuria (44.6\%), uremic encephalopathy (17.9\%) and hyperkalemia (16.1). Complete recovery was observed in $42.9 \%(n=24)$, partial recovery in $10.7 \%(n=6)$ and dialysis dependency in $8.9 \%(n=5)$. Death occurred in $12.5 \%(n=7)$. Severe anemia was significantly associated to death $(\mathrm{P}<0.005)$.

Conclusions: AKI with hemolytic anemia frequently occurred in young adults presenting with jaundice and fever. Malaria was the most common etiology. Dialysis was needed in most patients, and only half achieved complete renal recovery. 


\section{Abstract 7:}

\section{AKI PROFILE AND OUTCOMES IN A NEPHROLOGY UNIT IN SUB-SAHARAN AFRICA}

K Mukwelle ${ }^{2}$, M Mahamat $^{1}$, V Ndzana ${ }^{2}$, A Nono ${ }^{2}$, Dorothee Soh ${ }^{1}, \mathrm{G}$ Ashuntantang ${ }^{1}$

${ }^{1}$ Department of Internal Medicine and Specialties, Faculty of Medicine and Biomedical Sciences, University of Yaoundé 1, Yaoundé, Cameroon; ${ }^{2}$ Hemodialysis Unit, Yaoundé General Hospital, Yaoundé, Cameroon.

Objectives: To determine the outcome of patients with AKI admitted into the nephrology unit of the Yaoundé general hospital, Cameroon

Methods: We carried out a prospective, descriptive study from September 2017 to March 2019. We recruited all patients admitted into the nephrology unit of the Yaoundé general hospital. We excluded patients with pre-existing CKD. The diagnosis of AKI was made if risk factors for AKI were present, and at least two values of creatinine within the month of diagnosis of AKI. Patients were staged for AKI using the KDIGO 2012 criteria. Outcome was noted at discharge and at 3 months.

Results: We recruited 58 adults and 11 children. Among adults, 60.3\% (35) were men, Mean age among adults was $37.42 \pm 19.42$ years. About $95 \%$ of patients had community acquired AKI. Up to $88 \%(61)$ were classified KDIGO stage III, $8 \%$ (6) stage II, 3\% (2) stage I. Kidney injury was tubular in 68\% (47), glomerular in 24\% (17), vascular in 7\% (5). Obstetric AKI was seen in 4.3\% (3). Diarrhoea and vomiting were the most frequent precipitating factors for AKI $53.6 \%$ (37). A total of 48 patients (69.5\%) had indication for dialysis. Amongst these, the most frequent indication was uremia in $43 \%$ (21), followed by anuria in 35\% (17). Dialysis was done in $95.8 \%$ (46) of 48 patients requiring dialysis. Due to financial constraints, 2 patients could not afford hemodialysis. Complete renal recovery was noted in 32\% (22) of patients on discharge and in $75.4 \%$ (52) at 3 months. Death was noted in $18.8 \%$ (13), $2.9 \%$ (2) were lost to follow up and $2.9 \%$ (2) progressed to CKD.

Conclusions: Most cases of AKI require hemodialysis. Despite access to hemodialysis, mortality from AKI remains high and inaccessibility to treatment remains present. Both renal and patient outcome are generally good and similar to those reported in other developed countries.

\section{Abstract 8: \\ CHRONIC KIDNEY DISEASE IN SICKLE CELL DISEASE: PREVALENCE AND ASSOCIATED FACTORS IN THE ECONOMIC CAPITAL OF CAMEROON}

Hermine Danielle Fouda Menye epouse Ebana

General Hospital of Douala, Faculty of Medicine and biomedical Sciences of Yaoundé- University of Yaoundé I.

Hermine D Fouda ${ }^{1,2}$, Gilles Wandji ${ }^{1}$, Denis G Teuwafeu ${ }^{3}$, Marie Patrice Halle ${ }^{1,4}$, Alex Mambap T ${ }^{5}$, Hilaire Djantio ${ }^{4}$, Francois F Kaze ${ }^{2,6}$, Gloria Ashuntantang ${ }^{2,7}$

${ }^{1}$ General Hospital of Douala; ${ }^{2}$ Faculty of Medicine and Biomedical Sciences of Yaoundé, University of Yaoundé I, ${ }^{3}$ Faculty of Health Sciences - Buéa; ${ }^{4}$ Faculty of Medicine and Pharmaceutics Science-Douala; ${ }^{5}$ Regional Hospital of Bamenda; ${ }^{6}$ Teaching Hospital of Yaoundé; ${ }^{7}$ General hospital of Yaoundé.

Background: Previous studies in Cameroon have reported a high prevalence of proteinuria (93\%) and urinary abnormalities (78\%) in sickle cell anemia (SCA). However, the exact prevalence of chronic kidney disease (CKD) in SCA is not known since the persistence of these anomalies at 3 months was not evaluated. Moreover, the prevalence of CKD in people with Sickle cell trait (SCT) in Cameroon has not been studied.

Objective: Evaluate the prevalence of CKD in people with sickle cell disease in the main SCA health facility of the economic capital of Cameroon.

PATIENTS AND METHOD: SCA patients were recruited during routine visits between April $1^{\text {st }}$ and September $30^{\text {th }} 2017$ at Douala Laquintinie hospital. SCT patients in the family (parents or siblings) of homozygote patients were also recruited. CKD was diagnosed and classified according to 2012 KDIGO criteria. Only patients with persistent urinary anomalies or decreased eGFR at 3 months were considered to have CKD.

Results: A total of 107 patients including 81 SCA (men 62\%) and 26 SCT (men 46\%) participated in this study. SCA patients were younger (17 years Vs 35 years, $p<0.001$ ) with lower BMI, systolic and diastolic BP. Overall, CKD was found in 16 patients (15\%): 13 patients (16\%) in the SCA group and $3(11 \%)$ in the SCT group. CKD frequency was 
comparable in the both groups $(p=0.76)$. Albuminuria was more common in the SCA group $(12 \neq 1$ patient, $p=0.018)$. Age $\leq 25$ years was protective factor for both CKD $(O R=0.20[0.003-0.135], p<0.001)$ and albuminuria $(O R=0.23$ [0.004-0.124], $p<0.001$ ) in SCD group.

Conclusion: CKD is less prevalent in our setting compared to previous studies in Cameroon and sub-Saharan Africa.

\section{Abstract 9: \\ EVOLUTIONARY PROFILE OF ACUTE KIDNEY INJURY IN NEPHROLOGY AND HEMODIALYSIS AT THE YALGADO OUEDRAOGO UNIVERSITY HOSPITAL CENTER (CHU-YO)}

NITIEMA I, COULIBALY G, SOME CL, LENGANI HA, BONZI J, SANOU G, LENGANI A.

Objectives: Acute kidney injury (AKI) is a common syndrome that can be complicated by chronic kidney disease (CKD), which is a real public health problem. Through this study we want to determine the evolutionary profile of the AKI at CHU-YO.

Methods: A retrospective study was conducted on medical data from January 1, 2014 to December 31, 2016. All patients aged 15 years and over hospitalized for AKI (KDIGO 2012 definition) in the Nephrology and Hemodialysis Department of the CHU-YO were included in the study. Clinical and paraclinical data were collected.

Results: The study involved 68 patients, or $8.1 \%$ of admissions to the department during the study period. The sex ratio was 1.3 and the mean age was $38.7 \pm 17$ years. AKI was organic in $79.9 \%$ of cases. Acute tubular necrosis accounted for $90.8 \%$ of organic AKIs. AKI was classified at stage 3 of severity in $88.2 \%$ of cases. The mean duration of follow-up of the patients was $222.9 \pm 281.7$ days. Thirteen patients, $19.1 \%$, had at least six months of follow-up. Renal function was recovered in $55.9 \%$ of patients. CKD was observed in $11.9 \%$ of patients followed.

Conclusion: A high proportion of AKI is complicated by CKD.

\section{Abstract 10:}

\section{RENAL MANIFESTATION OBSERVED BY URINARY DIPSTICK IN SURVIVORS OF EBOLA VIRUS DISEASE}

Balde $\mathrm{MS}^{1}$, Diakité $\mathrm{F}^{1}$, Bah $\mathrm{AB}^{1}$, Bah $\mathrm{MK}^{1}$, Kaba $\mathrm{ML}^{1}$

${ }^{1}$ Nephrology-Hemodialysis service, Hospital Donka, Guinea.

Introduction: A survivor of the Ebola virus disease is defined as anyone who has a positive result in the RT-PCR test that can detect the virus in a biological fluid. In Guinea, there are 1270 survivor of EVD including 235 in Conakry. In the April 2016 WHO report showed sequelae of EVD survivor have been reported, including rheumatologic complications such as osteoarthritis, arthritis, eye complications, hearing complications, abdominal pain and neurological complications. The goal was to look for renal manifestations in cured EVD.

Methods: This was a 7-month prospective study of EVD survivor who agreed to participate in the study. We used a urine test dipstick to look for early signs of kidney disease in the survivor.

Results: We observed 70 people who were cured from EVD a total of 235 in Conakry, the frequency was $29.79 \%$. The average age was 30,92 years \pm 10.84 . The main risk factors found were smoking $13(22.8 \%)$, traditional selfmedication 6 (10.5\%); diabetes 4 (7\%) and hypertension 4 (7\%). Proteinuria was found in 57 patients.

Conclusions: In this study, proteinuria associated with hematuria represent the signs of kidney disease in a survivor EVD. The main risk factors observed were smoking, traditional self-medication, but also diabetes and hypertension. 


\title{
Abstract 11: \\ EVOLUTION OF AETIOLOGY OF ACUTE KIDNEY DISEASE FROM 1982 TO 2011 AT THE UNIVERSITY TEACHING HOSPITAL OF YOPOUGON
}

\author{
TIA WEU MELANIE, YAO HUBERT, LAGOU DELPHINE, TOURE AHMED KADIATA, WOGNIN MANAN ANNASTASIE, \\ OUATTARA BOURHAIMA, GNIONSAHE DAZE APOLLINAIRE \\ Teaching Hospital of Bouaké, Cote d'Ivoire.
}

Background: The epidemiological profile of acute kidney failure (AKF) changed gradually from one country to another over the last thirty years. The objective of this work was to study, between January 1982 and December 2011, the evolution of the epidemiology and etiology of the AKF in a nephrology service in Abidjan.

Methods: It was a retrospective study that included all patients admitted to the nephrology ward of the university teaching hospital of Yopougon in Abidjan for a creatinine $<14 \mathrm{mg} / \mathrm{l}$, with no history of diabetes, high blood pressure, HIV infection, chronic kidney disease or uropathy.

Results: During the study period (30 years), 1120 cases of AKF were included. Among these patients, they were 576 men versus 544 women with a sex ratio of 1.05 for men. The median age was 39 years. According to the ward of provenance, we noticed $70.17 \%, 19.28 \%$ from antenatal center and $9.55 \%$ of operating room service. Obstetrical and surgical AKF cases decreased respectively from $41.4 \%$ and $22.5 \%$ in 1982 to $5.1 \%$ and $6.8 \%$ in 2011 . Otherwise, there was a doubling of the medical IRA frequency from 1982 (38.9\%) to 2011 (88.1\%). The main etiology of medical AKF was bacterial and viral infections representing $30.2 \%$. Mortality was $22 \%$, mainly from obstetrical (31.9\%) and surgical (28.1\%) causes. The incidence of medical conditions at the origin of death was $18.4 \%$.

Conclusions: AKF is common and concerns young male subjects in particular. A decrease of the obstetrical and surgical AKF and a progression of the medical cases have been observed. Mortality remains high and calls for effective measures to reduce this mortality rate.

\section{Abstract 12: \\ CLINICAL PATTERN AND OUTCOMES OF ACUTE KIDNEY FAILURE PATIENTS ADMITTED IN EMERGENCY ROOM IN A TERTIARY HOSPITAL OF BOUAKÉ IN IVORY COAST}

TIA WEU MELANIE, WOGNIN MANZAN ANASTASIE, TOURE AHMED KADIATOU, MEY NATANAEL, KOUAME GILLES, OUATTARA BOURHAIMA, GNIONSAHE DAZE APOLLINAIRE

University Teaching Hospital of Bouaké, Alassane Ouattara University, Ivory Coast.

Background: The etiologies and outcome of acute kidney injury (AKI) in resource-limited countries are largely related to poverty. Mortality remains high. We sought to describe clinical pattern and outcomes AKI amongst patients in the University Teaching Hospital of Bouaké in Ivory Coast.

Methods: We retrospectively reviewed records of patients hospitalized in emergency room from January 1 to December 31, 2016. Diagnosis of AKI was done according AKIN criteria.

Results: During the study period, we collected 221 cases of acute kidney failure on a total of 1,778 cases, giving a prevalence of $12.4 \%$. The mean age was 54.19 years. Sex ratio of 1.4 was observed. We noticed hypertension in $40.4 \%$, heart disease $16.9 \%$ and diabetes in $14.3 \%$. Patients were classified in stage I in $63.8 \%$, stage II $22.1 \%$, and $14.1 \%$ in stage III according to AKIN. Functional acute kidney failure was seen in most of cases (93.2\%). Dialysis was indicated in $15(6,8 \%)$ patients but only $3(1,4 \%)$ patients effectively underwent the therapy. Mortality was $45.2 \%$ and 81 patients (36.6\%) were discharged. Mortality factors were creatinine more than $30 \mathrm{mg} / \mathrm{l}$ and sepsis.

Conclusions: Acute kidney failure is more common and it is seen in stage II (AKIN). Mortality remains high due to sepsis and creatinine more than $30 \mathrm{mg} / \mathrm{l}$. 


\section{Abstract 13:}

\section{TRENDS IN THE EPIDEMIOLOGY OF ACUTE KIDNEY INJURY IN COTE D'IVOIRE FROM 1982 TO 2012}

GUEI Monlet Cyr ${ }^{1}$, DELORG Diane Christelle ${ }^{1}$, YAO Kouame Hubert ${ }^{2}$, LAGOU Delphine Amélie ${ }^{1}$, WOGNIN Anasthasie ${ }^{1}$, KONAN Serge Didier ${ }^{2}$, ACKOUNDOU-N'GUESSAN Clément ${ }^{1}$, GNIONSAHE Daze Apollinaire ${ }^{1}$

${ }^{1}$ University Teaching Hospital of Yopougon, Abidjan, Côte d'Ivoire; ${ }^{2}$ University Teaching Hospital of Treichville, Abidjan, Côte d'Ivoire.

Introduction: Acute kidney injuries (AKI), occurring in diverse clinical settings and with a variety of etiologies, often progress to lethal complications.

Purpose: To investigate trends in the epidemiology of AKIs observed from 1982 to 2012 in Côte d'Ivoire so as to suggest prevention and treatment strategies aimed at reducing mortality.

Methods: Retrospective descriptive and analytical study on the medical records of patients admitted for AKI in the department of nephrology and intensive care unit of the University Teaching Hospitals of Yopougon, Cocody and Treichville, and Polyclinique Internationale Sainte Anne Marie. AKI was defined as serum creatinine greater than 14 $\mathrm{mg} / \mathrm{l}$ for less than 3 months and classified as surgical, medical and gynecological-obstetric AKI according to the circumstances of onset.

Results: We counted a total of 1120 patients. The mean age was 33.9 years with a range of 2 to 91 years, and a sex ratio of 1.05 in favor of men. Summary table of results by decade:

\begin{tabular}{llll}
\hline & $1982-1991$ & $1992-2001$ & $2002-2012$ \\
\hline Total number & 381 & 462 & 277 \\
M/F sex ratio & 0.6 & 1.3 & 1.4 \\
Mean age (years) & $28.7 \pm 11.7$ & $32.6 \pm 13.4$ & $40.1 \pm 16.6$ \\
Type of AKI & & & \\
Gynecological-obstetric & $40.7 \%$ & $9.6 \%$ & $5.1 \%$ \\
Medical & $37.3 \%$ & $85 \%$ & $88.1 \%$ \\
Surgical & $22 \%$ & $5.4 \%$ & $6.8 \%$ \\
Diuresis & & $12.2 \%$ & $14.8 \%$ \\
Anuric & $24.4 \%$ & $87.8 \%$ & $85.2 \%$ \\
Preserved & $75.6 \%$ & $20.3 \%$ & $62.7 \%$ \\
Hemodialysis & $39.8 \%$ & $10 \%$ & $15 \%$ \\
Outcome (Death) & $58 \%$ & & \\
\hline
\end{tabular}

\section{Conclusions:}

AKI was common and affecting patients of all ages, preferably young people and adults. The prevalence of medical AKIs increased over the last three decades. Conversely, that of surgical, gynecological and obstetrical AKIs decreased. The frequency of hemodialysis use increased over the last three decades. However, in spite of the progress made in AKI treatment, its overall mortality was significant. AKI remains a public health problem requiring appropriate preventive and curative measures to reduce its incidence and mortality.

\section{Abstract 14:}

\section{ENTERIC FEVER CAUSING HEMOLYTIC UREMIC SYNDROME}

Introduction: Hemolytic uremic syndrome presents with a classic triad of microangiopathic hemolytic anemia, thrombocytopenia and acute kidney injury. It is usually caused by toxigenic strains of E coli and Shigella dysenteriae which produce the shiga toxin. It can also be caused by Streptococcus pneumonia and complement disorders. Typhoid fever causing hemolytic uremic syndrome is quite rare.

Case presentation: A 54 year old male presented with a seven day history of diarrhea. He had been started on ciprofloxacin after a stool test for salmonella antigen was positive. He presented after seven days with worsening of diarrhea and had developed vomiting. Examination revealed mild dehydration with normal vital signs and tenderness in the right and left iliac fossa regions. His laboratory investigations showed an elevated urea and creatinine with a low potassium. Blood cultures done on the day of admission grew $S$ typhi sensitive to ciprofloxacin. The platelets and hemoglobin dropped on the second day of admission. His lactate dehydrogenase was markedly elevated and a 
peripheral blood film showed schistocytes. He was started on antibiotics, hemodialysis and potassium supplementation and had complete recovery of his renal function and blood parameters after seven days.

Discussion: Typical hemolytic uremic syndrome occurs secondary to infection by shiga-toxin producing E coli and less frequently due to $S$ dysenteriae. It can also be caused by Streptococcus pneumonia. Salmonella typhi is not a common cause of hemolytic uremic syndrome and there are few case reports in the literature of salmonella induced HUS. The inciting toxin in hemolytic uremic syndrome secondary to $\mathrm{S}$ typhi has not yet been identified. The treatment is mainly supportive comprising appropriate antibiotics, correcting fluid and electrolyte imbalances and dialysis when required.

\title{
Abstract 15: \\ IN-HOSPITAL MORTALITY OF ACUTE KIDNEY INJURY IN PATIENTS WITH HIV: A 2-YEAR RETROSPECTIVE COHORT STUDY
}

MY Chothia, N Ramsunder

Division of Nephrology, Department of Medicine, Faculty of Medicine and Health Sciences, Stellenbosch University and Tygerberg Hospital.

Background: Acute kidney injury (AKI) in HIV patients in Sub-Saharan Africa is common. There is a paucity of epidemiological data regarding the outcome of AKI in those with and without HIV from the African continent. Objective: The primary outcome was to determine the overall in-hospital mortality of AKI.

Methods: A single centre study of all consecutive adult patients with AKI referred to the renal unit at Tygerberg Hospital from 1 January 2015 to 31 December 2016. AKI was defined as a recent normal serum creatinine (<3 months) and/or normal kidney sizes on ultrasound (> $100 \mathrm{~mm}$ length). Those with proteinuria $>3.0 \mathrm{~g} /$ day were excluded.

Results: We identified a total of 291 patients with AKI of which $39.9 \%$ were HIV positive. Overall, 31\% patients died of which $34.5 \%$ were HIV positive and $29.1 \%$ were HIV negative $(p=0.34)$ (figure 1$)$. At hospital admission, more HIV positive patients had tuberculosis $(p<0.01)$ and had higher admission serum creatinine $(p<0.01)$. Of those that died, the HIV positive patients were younger $(p<0.01)$, were predominantly Black $(p<0.01)$ and were predominantly admitted to medical wards $(p<0.01)$. There was no difference in mortality regardless of renal replacement therapy ( $p$ $=0.50)$. Logistic regression identified Mixed race (OR 2.47, $p=0.02), \operatorname{HIV}(O R 2.69, p<0.01)$ and surgical ward admission (OR 2.05, $\mathrm{p}=0.03$ ) as predictors for death.

Conclusions: Overall in-hospital mortality of AKI was high. HIV was associated with a greater risk of death that may be the result of late presentation of both the AKI as well as the HIV.

\section{Abstract 16: \\ EPIDEMIOLOGICAL AND ETIOLOGICAL ASPECTS OF ACUTE RENAL FAILURE (ARF): ABOUT 90 CASES IN NEPHROLOGY HOSPITALIZATION OF SYLVANUS OLYMPIO UNIVERSITY HOSPITAL IN TOGO}

\author{
Dolaama B, Sabi KA, Amekoudi EYM, Ali IM, Tona KG, Tsevi YM, Attisso AE, Noto-Kadou-Kaza B \\ Nephrology and Haemodialysis Service of Sylvanus Olympio Teaching Hospital, Lome, Togo.
}

Objectives: To describe the epidemiology of ARF and to specify the prognosis for nephrology hospitalization at Sylvanus Olympio University Hospital, TOGO.

Methodology: This was a retrospective descriptive and analytical study in the nephrology and haemodialysis department of Sylvanus Olympio University Hospital. It covered all patients hospitalized for ARF during the period from January 1, 2015 to December 31, 2016 (02 years). The ARF was selected according to KDIGO criteria and retrospective criteria.

Results: 90 patients with acute renal failure were included in our study, an absolute prevalence of ARF of $15.8 \%$. The mean age was $40.8 \pm 19.3$ years with a male / female sex ratio of 1.2 . The majority of patients (85.6\%) had low socioeconomic status. Among the antecedents, the HTA was majority (42.2\%) and the patients were under Nonsteroidal anti-inflammatory drug in 34\% of the cases before their admission. The mean serum creatinine was $96.3 \mathrm{mg}$ / I [IQR $=18.1-263 \mathrm{mg} / \mathrm{l}]$, the mean haemoglobin level was $8.88 \mathrm{~g} / \mathrm{dl}[\mathrm{IQR}=2.8-14.7 \mathrm{~g} / \mathrm{dl}]$ and the mean serum calcium level was $90.5 \mathrm{mg} / \mathrm{I}$ [IQR $=70-177 \mathrm{mg} / \mathrm{l}]$. The ARF was organic in $73.3 \%$ of cases with a probable NTA representing $54.4 \%$ of organic ARF. Haemodialysis was performed in $12.2 \%$ of patients and death occurred in $12.2 \%$ of these patients with septic shock as the main cause (63.6\%). According to univariate analysis, ARF was significantly associated 
$(p<0,05)$ with haemoglobin levels and the comparative serum creatinine $(p<0,05)$ was significantly associated with mortality.

Conclusion: ARF is an increasingly common pathology and therefore represents a real public health problem. Early detection of risk situations and the selective use of appropriate protective measures allow rapid restoration of renal perfusion.

\title{
CHRONIC KIDNEY DISEASE
}

\section{Abstract 17: \\ COST OF THE MANAGEMENT OF CHRONIC KIDNEY DISEASE IN THE NEPHROLOGY DEPARTMENT OF THE UNIVERSITY HOSPITAL YALGADO OUÉDRAOGO (BURKINA FASO)}

Habin'Yabama Aida LENGANI', Patricia OUÉDRAOGO'1, Mouna Yéri MJ. OUÉDRAOGO ${ }^{2}$, Gaoussou SANOU', Joel Ignace NITIÉMA ${ }^{1}$, Gérard COULIBALY ${ }^{1}$

${ }^{1}$ Departement of nephrology, University Hospital Yalgado Ouédraogo, Ouagadougou, Burkina Faso; ${ }^{2}$ Workers' Health Office, Ouagadougou, Burkina Faso.

Objectives: To study the costs incurred by patients suffering from chronic kidney disease (CKD) in Ouagadougou. Methods: We conducted a cross-sectional partial economic valuation study based on costs from June 15 to August 14 , 2017. All patients with a stage 3 to 5 CKD who were followed up during the study period and who gave their consent were included.

Results: A total of 169 patients (51.5\% men) were enrolled. The mean age of $53.0 \pm 17.2$ years. Among the patients, $44.4 \%$ had a monthly income of less than 30,000 FCFA (52.53 US\$). For ambulatory patients (73.4\%), the average

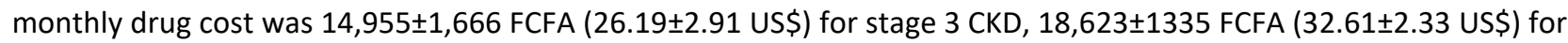
stage 4 and $29,493 \pm 4,088$ FCFA (51.65 \pm 7.15 US\$) for stage 5 . The average cost of paraclinical examinations per

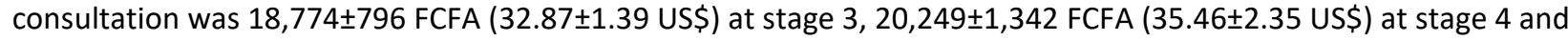
$21,537 \pm 2,275$ FCFA ( $37.71 \pm 3.98$ US\$) at stage 5 . The overall average cost of managing hospitalized stage 5 CKD patients was $207,901 \pm 37,337$ FCFA (364.09 \pm 65.38 US\$) for the 9 who had not started hemodialysis and $551,813 \pm 33,016$ FCFA $(966.39 \pm 57.82$ US\$) for the 45 who had started hemodialysis. The average loss of income per patient was estimated at $37,225 \pm 9182$ FCFA ( $65.19 \pm 16.08$ US\$). In 53.8\% of cases, families contributed to the care. Conclusion: The costs of managing CKD are beyond the financial resources of the majority of patients. Health insurance systems should be put in place to solve this problem.

[On average, 1 US\$=571.01 FCFA during the study period.]

\section{Abstract 19:}

\section{PROGRESSION FACTORS OF CHRONIC RENAL DISEASE IN THE NEPHROLOGY DEPARTMENT OF THE YALGADO OUÉDRAOGO UNIVERSITY HOSPITAL CENTRE IN OUAGADOUGOU}

\author{
G. Coulibaly, J.Y. BONZI, D. DJIGMA, A. Y. LENGANI, H. TRAORE, G. SANOU, I. J. NITIEMA, A. LENGANI \\ Nephrology-hemodialysis departement of yalgado OUEDRAOGO University Hospital
}

Objective: The progression of chronic kidney disease (CKD) to stage 5 is influenced by a variety of factors. These factors may be related to the patient's habits and lifestyle, initial nephropathy, co-morbidities or medicines. We report the progression factors of chronic kidney disease in a cohort of patients followed in a nephrology department. Method: We reviewed the records of patients over the age of 18 years followed in outpatient clinics for CKD from January 1, 2014 to December 31, 2017. Dialysis and kidney transplant patients were not included. The CKD was defined by the existence of markers of renal impairment for at least three months. In the absence of histological analysis, we defined presumed nephropathies on the basis of clinical and paraclinical criteria. From January 1, 2014 to December 31, 2014, 123 patients were seen in first consultations for CKD, 57 of whom (46.3\%), had regular follow-up until December 31, 2017 and 66 patients were lost to follow-up.

Results: the average age was $55.5 \pm 16$ years, there was no significant difference between men and women; the sex ratio was 1.9. Patients over 60 years of age represented $45.5 \%$. The socio-economic level was low in 81 patients or $65.9 \%$ of cases. The initial suspected nephropathies were as follows: chronic vascular nephropathy: 64 cases (52\%); 
chronic glomerulonephritis: 46 cases (37.4\%); chronic tubulointerstitial nephropathy: 9 cases (7.4\%); HIV-associated nephropathy: 3 cases (2.4\%); polycystosis: 1 case $(0.8 \%)$. High blood pressure, diabetes, traditional medication and non-steroidal anti-inflammatory drugs were found in 95 cases $(77.8 \%), 26$ cases $(21.1 \%), 35$ cases $(28.5 \%)$ and 41 cases (33.3\%) respectively. At first contact with the nephrologist, the average glomerular filtration rate was $30.4 \pm 18$ $\mathrm{ml} / \mathrm{min} / 1.73 \mathrm{~m}^{2}$. Thirty patients (24.4\%) had reached stage 5 immediately. In univariate analysis, the progression factors of the CKD were: age, provenance, a history of bilharzia, existence of acute oliguria or initial lung edema, anemia syndrome and non-steroidal anti-inflammatory drugs.

Conclusion: The progression of CKD in our context is influenced by socio-demographic, clinical and drug-related factors.

\section{Abstract 20:}

\section{TUBEROUS SCLEROSIS (OR BOURNEVILLE'S DISEASE): FIRST CASE DESCRIBED IN CHAD IN A YOUNG LADY OF 27 YEARS OLD}

IBRAHIM Hamat (1), Aboubakar Abdelkerim Mahamat(1), MAHAMAT Youssouf (2), MAHAMAT Abderraman Guillaume (3), TALL Ahmed Lemrabott (4), Haoua YOUSSOUF SAID (4), Charfadine Senoussi Abdelkerim(1), Nassima hissein abdelaziz(1), MAHAMAT ABAKAR Hapsita (1), ALI Hissein Mahamat (3), KA Elhadj FARY (4), DIOUF Boucar (4) 1: Department of Nephrology-Haemodialysis, General Hospital of National Reference/ 130 N'Djamena, Chad; 2: Department of Internal Medicine, the Renaissance Hospital of N'Djamena, Chad; 3: Department of NephrologyHaemodialysis of the Renaissance Hospital of N'Djamena, Chad; 4: Department of Nephrology, Aristide Le Dantec Hospital of Dakar, Senegal.

Introduction: The Bourneville's disease also called the tuberous sclerosis is a rare genetic disorder with a prevalence of about 1 case per 6000 living births, inherited in an autosomal dominant way with a multi-systemic clinical expression. This disease is characterized by hamartoma growing and many other tumours mainly located in the brain, kidneys, skin, heart, bowel, liver, retina and lungs.

Observation: We reported a case of a young Chadian lady of 27 years old. She has consulted in the Nephrology Unit for a recurrent left renal colic for which the ultrasound has suggested the CACCI RICCI disease.

The questioning and clinical examination have observed a moderated autism syndrome, a recurrent renal colic since 12 years, some dermatological damages (angiofibroma, molluscum pendulum, hypomelanotic macules) and the CT urogram has showed multi-bilateral angiolipoma on the kidneys which suggested the Bourneville's disease. A treatment based on Sirolimus has been proposed.

Discussion: The final diagnosis of the Bourneville's disease associates two major criteria or a major and two minor criteria as described by Gomez. Our patient presents three major criteria from which the multi-bilateral angiomyolipoma without any effect on the kidney function.

Conclusion: The tuberous sclerosis or Bourneville's disease is a rare disease. Our study has confirmed it because this is the first case in seven years of consultations in the Nephrology department.

\section{Abstract 21: \\ PREVALENCE AND FACTORS ASSOCIATED WITH RENAL IMPAIRMENT AMOUNT HIV INFECTED PATIENTS RECEIVING TENOFOVIR CONTAINING ANTIRETROVIRAL REGIMEN: THE CASE OF THE REGIONAL HOSPITAL OF THE SOUTH WEST CAMEROON}

Denis Georges Teuwafeu, Victoire Fotsing, Marie Patrice Halle, Gloria Ashuntantang

Background: Although uncommon, renal impairment attributable to TDF has been reported among HIV infected patients receiving TDF containing ART in developed countries. Given the widespread use of this regimen in Cameroon, little is known about the proportion of renal impairment attributable to TDF toxicity in our setting.

Method: This was a hospital based cross-sectional study on HIV infected patients who had started TDF-based regimen for a minimum of three months. Data were obtained by patient's interview, patient's records review and physical examination. Blood and spot urine sample were collected to perform urine dipstick, blood glucose, urinary and serum creatinine and phosphate. Glomerular filtration rate (GFR) was estimated using modification of diet in renal disease (MDRD). Renal impairment was defined as eGFR<60ml/min and/or proteinuria and/or proximal tubular dysfunction. 
Proximal tubular dysfunction was defined as having a FePO $<18 \%$ and proteinuria. Multivariate analysis was done to identify independent association.

Results: Of 240 patients included in the study, 38.8\% developed renal impairment of which 115 (47.9\%) had proteinuria, $11.6 \%$ ( 28 out of 240 ) eGFR $<60 \mathrm{ml} / \mathrm{min}$ and $0.83 \%$ ( 2 out of 240 ) proximal tubular dysfunction. The proportion of renal impairment attributable to TDF toxicity was $2.15 \%$ ( 2 out of 93 ). No factor was independently associated with renal impairment.

Conclusion: The prevalence of renal impairment among HIV infected patient receiving TDF containing therapy at the Buea and Limbe Regional Hospital is high with $2.15 \%$ of renal impairment been attributable to TDF toxicity. Therefore, patients should be monitored using both eGFR end FePO.

\title{
Abstract 22:
}

\section{ASSESSMENT OF KNOWLEDGE, ATTITUDES AND PRACTICES ON CHRONIC KIDNEY DISEASE AMONG PATIENTS WITH DIABETES MELLITUS}

\author{
Denis Georges Teuwafeu, Ngomba Maryl Ngowo, Francois Folefack Kaze, Simeon-Pierre Choukem
}

Background: Appropriate information and practices of CKD amongst patients with diabetes are mandatory for prevention of diabetes related kidney disease. Unfortunately, clinicians often forget to educate patients on proper knowledge, attitudes and practices of CKD.

Objective: This study aimed at assessing the knowledge, attitudes and practices (KAP) on CKD and its prevention among patients with diabetes mellitus and factors associated with various levels of KAP in the Southwest Region. Methodology: this hospital-based cross-sectional included all consenting patients with diabetes mellitus at various diabetes out-patient and in-patient units of three regional hospital. Data was collected using semi-structured questionnaires. Statistical significance was set at $\mathrm{P}<0.05$ and data was analysed using Epi-info version 7.2. Results: We included 405 participants, 97\% ( $n=391)$ had type 2 diabetes. the mean age was 54.49(14.15). Females patients were more represented 259 (64\%). Duration of diabetes ranged from 6-432 months. Majority of the participants had moderate to good knowledge (46-43\%) on CKD. A good number of participants had good attitudes towards CKD and its prevention. Most patients were willing to seek health care from various sources should they develop CKD. Factors like age, level of education were significantly associated with level of knowledge (Association between age and level of knowledge $\mathrm{P}<0.04$, association between level of education and knowledge $\mathrm{P}<0.002$ ). Conclusion: The knowledge on CKD among patients with diabetes is moderate and it was strongly associated with level of education and age. Patients had positive attitudes and good practices.

\section{Abstract 23:}

\section{CONGOLESE POPULATION: LEVEL OF KNOWLEDGE AND INFORMATION ABOUT KIDNEY DISEASE}

Mahoungou G, Eyeni Sinomono T, Botokoto H, Mobengo L, Loumingou R. Nephrology Department of University-affiliated hospital of Brazzaville (CHU), Congo.

The prevalence of kidney disease cases is increasing in Congo and worldwide, the chronic kidney failure resulting from the disease usually requires cumbersome and costly methods of treatment. Diagnosis and early care of kidney disease are therefore essential to delay or avoid the use of extra-renal methods of treatment to which access is still limited in Congo. To date, no awareness campaign to educate the Congolese general public on kidney diseases has yet been conducted.

We report the results of a public survey aimed at assessing the level of knowledge and information of a representative sample of the population on this topic. Questions included knowledge of kidney function, severity of kidney disease, perception of kidney disease symptoms, and associated therapies.

This study shows that Congolese are not familiar with the anatomical location of the kidneys and their roles. They are not familiar with the main symptoms associated with kidney disease. The different therapeutic methods in case of chronic kidney failure are not well known. The interviewees think more about transplant, or even death than treatment by dialysis. The distinctive aspects of the latter are poorly known since some think it is done on a monthly basis. 
A real discrepancy exists between the severity of chronic kidney disease and the level of knowledge and information of the general public. This situation contributes to the late care of patients with chronic kidney disease. Public awareness is therefore a vital prerequisite.

\title{
Abstract 24:
}

\section{ESTIMATING TIME FOR KIDNEY DISEASE MANAGEMENT IN HOSPITAL DONKA}

\author{
Balde MS, Diakité F, Bah AB, Traoré M, Haba S, Kaba ML, Bah AO
}

Service of Nephrology in Teaching hospital of Donka.

\begin{abstract}
Introduction: Chronic kidney disease (CKD) is defined as the presence, for more than three months the markers of renal impairment or a decreased glomerular filtration rate (GFR) below to $60 \mathrm{ml} / \mathrm{min}$. $1.73 \mathrm{~m} 2$. The purpose of our study was to determine the management time of CKD.

Materials and Methods: This was a descriptive study lasting three months. We included in this study, patients diagnosed with CKD hospitalized in the nephrology ward. The management Time of the CKD was evaluated in months. Results: During our period, 75 patients presented with CKD. The average age was $31.6 \pm 16.93$ years old. The average duration of hospitalization was 29 days \pm 2.69 days. Discussion: The duration of hospitalization was large, in our conditions the diagnosis of CKD is late, patients are treated with complications related to uremia whose management is cumbersome and therapeutic. interest in developing an early diagnosis strategy in order to limit the complications of the uremic state, the life-threatening condition of which is still threatened. Conclusion: the time in the management of the CKD is larg, this time should be shortened through an early diagnosis thus avoiding the complications of uremia to reducing the admission on dialysis.
\end{abstract}

\section{Abstract 25: \\ FACTEURS DE PROGRESSION DE L'INSUFFISANCE RÉNALE CHRONIQUE PRÉTERMINALE AU CHU DE DONKA \\ FACTOR PROGRESSION OF RENAL INSUFFICIENCY BEFORE END STADE OF KIDNEY DISEASE IN TEACHING HOSPITAL OF DONKA}

\author{
F. Diakité, AB Bah, MS Baldé, M Traoré, D Djibrine, I Chérif, ML Kaba, AO Bah \\ Néphrologie-hémodialyse, $\mathrm{CHU}$ de Donka
}

Introduction: The objective of our study was to determine the factors of progression of kidney disease before end stage renal insufficiency in Teaching Hospital of Donka.

Patients and methods: It was an exploratory descriptive and analytical study we realized over four (04) months period going from the $1^{\text {th }}$ November 2017 with to $1^{\text {th }}$ March 2018. All the patients were included who having a clearance of the creatinine ranging between 15 and $29 \mathrm{ml} / \mathrm{min}$ during the period of study. We used qualitative and quantitative variable. The worsening of the renal function was appreciated by the rhythm of decreasing of the glomerular filtration rate (GFR) estimated by the calculated clearance of the creatinine according to MDRD formula. An analysis of multivariable logistic regression was used to analyze the factors of progression of the renal disease before end stage disease.

Results: The results of the univariable analysis show that the average age of the patients who presented a decline of the renal function $(61,69 \pm 16,30)$ was significantly different from that of the patients who did not have decline $(46,74 \pm 20,56)$ of the renal function, $(p=0,022)$. Also, the patient who have hyperuricemia $(57,1 \%)$ is significantly different from that of the patients not having decline of the renal function (42,9\%). The results of the multivariable analysis show that the patients who presented a hyperuricemia had 9,42 times more risk to be declining renal function (OR=9,42; IC to $95 \%$ : [1,37-64,48].

Conclusion: The ignorance of factors progression by the patients in chronic renal insufficiency before end stage kidney disease would explain their high frequencies. 


\section{Abstract 26:}

\section{RENAL INVOLVMENT IN MONOCLONAL GAMMOPATHIES IN THE TEACHING HOSPITAL OF YOPOUGON}

TIA Weu Mélanie, Touré Ahmed Kadiata, Sawadogo Amidou, Wognin Manzan, Ouattara Bourhaima, GnionsahéDaze Apollinaire

Service de Néphrologie, CHU de Yopougon

Background: Renal involvement are common during monoclonal gammopathies and their occurrence affects the survival of patients. Our objective was to describe these renal features from 2004 to 2016 at the teaching hospital of Yopougon in Abidjan.

Methods: renal failure was defined as $20 \mathrm{mg} / \mathrm{L}>$ creatinine and or proteinuria $>500 \mathrm{mg} / 24$ hours.

Results: We identified 42 cases of GM including 40 cases of multiple myeloma and 2 cases of monoclonal gammopathy of underdetermined significance. The rate of renal impairment was $57 \%(n=24)$. These were 3 cases of glomerular nephropathy and 20 cases of tubular disease. Renal biopsy was performed in two cases and it was one case of cast nephropathy and one case of Randall's disease. The factors associated with renal impairment were mainly hypercalcemia ( 23 cases) and hyperuricemia ( 24 cases). Three cases were treated by hemodialysis. There were 6 $(14.3 \%)$ deaths among patients with renal impairment.

Conclusion: Tubular injury was common among patients with monoclonal gammopathy. It was associated with hypercalcemia and Hyperuricemia.

\section{Abstract 27:}

\section{KIDNEY REGISTRY OF CÔTE D'IVOIRE (2RCI): IMPLEMENTATION}

Yao $\mathrm{KH}^{1}$, Moudachirou $\mathrm{MIA}^{1}$, Traore $\mathrm{AN}^{2}$, Mama Djima $\mathrm{M}^{3}$, Gnionsahe DA ${ }^{1}$

${ }^{1}$ Société Ivoirienne de Néphrologie; ${ }^{2}$ Altesse Data Consultant; ${ }^{3}$ Institut Pasteur de Côte d'Ivoire.

Context: In Côte d'Ivoire $(\mathrm{Cl})$ as in other African countries, there is an increase in the number of patients with end stage chronic kidney disease (ESCKD), with a major socio-economic impact. The absence of national data causes a lack of adequacy between supply of care and number of patients, making care difficult. Thus, an ESCKD registry is necessary.

Objectives: To generate epidemiological, clinical, therapeutic and outcomes data on ESCKD. This will help plan the delivery of healthcare services, therefore improve the clinical management and patients' quality of life.

Design: The conception of the Kidney Registry of $\mathrm{Cl}$ (2RCl) started in 2016. By June 2018, in collaboration with other kidney organizations such as the agency of Biomedicine of France, the terms of reference, data collection guide and several data collection forms were developed. A literature search was conducted to assist in the development of information leaflets and consent forms.

Electronic Registry: The $2 \mathrm{RCl}$ is accessible via a secure web portal where structured data are recorded in a SQL database. Nephrologists can only access it with a password. With a data entry guide and a data dictionary, data collection presents minimal risk to patients. The portal facilitates comparisons and aggregation of data; epidemiological and clinical reports/indicators will be generated.

Conclusion: The implementation of $2 \mathrm{RCl}$ has been a collaborative process that should have a substantial impact on the practice of nephrology. The data collected will help optimize the provision of healthcare services to patients with ESCKD. 


\author{
Abstract 28: \\ SEROPROTECTION FOR HEPATITIS B IN PATIENTS WITH NEPHROTIC SYNDROME \\ Elassas H,Chettati M, Fadili W, Laouad I. \\ Nephrology-Hemodialysis departement, Cadi Ayyad university, CHU Mohammed VI, Marrakech, Morocco
}

Introduction: Patients with nephrotic syndrome have been shown to have lower seroconversion to various vaccines due to immune dysregulation, prolonged immunosuppressive treatment and recurrent prolonged proteinuria. The primary aim of this study was to determine hepatitis B surface anti-body (anti-HBs) titers in patients with nephrotic syndrome who had been previously vaccinated against hepatitis $B$.

Material and Methods: This cross-sectional study was conducted in the department of Néphrology in Mohammed VI university hospital between January 2017 and January 2019. Patients with nephrotic syndrome who tested negative for hepatitis B surface antigen and who had previously been vaccinated against hepatitis $B$, with the last dose being at least 1 month prior to being included in the study.

Results: The patient cohort comprised 40 Patients ( 21 males;17 females) of whom 22 (55\%) had minimal change disease; $10(25 \%)$ had membranous nephropathy and 5(12\%) had membranoproliferative glomerulonephritis and $3(8 \%)$ focal segmental glomerulosclerosis. Twenty-one (28\%) patients had received only steroids, while $10 \%$ also received other immunosuppressants. Of the 40 patients enrolled, 19 (48\%) and $39(21 \%)$ had an anti-HBs titer of $\geq 10$ $\mathrm{mIU} / \mathrm{mL}$ (seroprotected) and $<10 \mathrm{mIU} / \mathrm{mL}$ (unprotected), respectively. The mean titer among all patients was 50.58 $\mathrm{mIU} / \mathrm{mL}$. The seroprotection rates were $63.6 \%$ in patients not receiving immunosuppressants and $35.7 \%$ in subjects on immunosuppressants $(\mathrm{P}=0.016)$.

Conclusions: Based on our results, we conclude that patients on immunosuppressants are less likely to seroconvert with vaccination. A higher dose (double) of hepatitis $B$ vaccine should be used for vaccinating such patients. Anti-HBs titers should be monitored in patients post-vaccination, and a booster should be given if titers fall to $<10 \mathrm{mIU} / \mathrm{mL}$.

\title{
Abstract 29:
}

\section{THE VIABILITY OF PALLIATIVE CARE AS A TREATMENT OPTION FOR ELDERLY PATIENTS WITH END-STAGE RENAL DISEASE IN UMUAHIA, SOUTH-EAST NIGERIA; A PRELIMINARY REPORT}

\author{
Okwuonu $\mathrm{CG}^{1}$, Alole $\mathrm{LM}^{2}$, Ojimadu $\mathrm{NE}^{2}$, Oviasu $\mathrm{E}^{3}$ \\ 1. Nephrology unit, Department of Internal Medicine, Federal Medical Centre Umuahia, Abia State; 2. Department of \\ Family Medicine, Federal Medical Centre UmuahiaAbia State; 3. Nephrology unit, Department of Internal Medicine, \\ University of Benin Teaching Hospital Benin Edo State.
}

Introduction: The number of elderly patients developing end-stage renal disease (ESRD) is on the increase in Nigeria. With multiple co-morbid conditions, evidence is needed to inform decision making for or against dialysis among these patients.

Objectives: To compare symptom burden, quality of life, functional status and one-year survival between elderly patients managed with palliative care and haemodialysis (HD).

Methods: A cross-sectional analysis of patients from 60 years and above with ESRD who were treated with either haemodialysis or palliative care over a period of one year. The symptom burden, quality of life and functional status were assessed by standard validated methods. Survival was defined as time from diagnosis of ESRD to either death or been censored. Kaplan-Meier survival analysis and Log rank test was used to estimate and compare survival. Cox proportional hazard regression models were used to investigate the impact of independent variables on survival. Results: Haemodialysis was offered to 57 (75\%) while 19 (25\%) choose palliative (non-aggressive) renal care for various reasons. Patients who opted for palliative care had a significantly higher mean symptom burden score and lower quality of life scores than their counterparts on dialysis treatment $(p=0.760)$. There was no significant difference in mean functional status scores between the two groups $(p=0.837)$. The mean survival time for those on dialysis treatment (7.1 months) was slightly higher than mean survival time for those on palliative care (5.8 months) but there is no significant evidence of difference in survival times for both groups ( $\log$ rank $=1.2, p=0.27$ ).

Conclusion: Palliative care maybe a viable option for management of ESRD as it has comparable survival advantage with haemodialysis; albeit with a higher symptom burden and lower quality of life. 
NEUROPSYCHIATRIC MANIFESTATIONS OF PATIENTS WITH CHRONIC KIDNEY DISEASE IN SOUTH EASTERN NIGERIA

\author{
Okafor $\mathrm{UH}^{1}$, Ezeme $\mathrm{M}^{2}$, Edeh $\mathrm{SO}^{1}$ \\ 1. Renal unit, Dept of Medicicne, ESUT teaching hospital, Parklane Enugu; 2. Dept of Psychiatry, ESUT teaching hospital \\ Parklane.
}

Background: Chronic kidney disease has been associated with various neuropsychiatric disorders. This impacts negatively on the quality of life of the patient, affects the clinical course and mortality. The aim of the study is to evaluate the neuropsychiatric manifestations of patients with chronic kidney disease in south eastern Nigeria. Methodology: This is a cross sectional study of patients with chronic kidney disease who presented to the renal unit of ESUT teaching hospital Parklane Enugu from 1st November 2018 to 30th April 2019. Consecutive patients who consented for the study were recruited. Their biodata and clinical parameters were documented. Diagnosis of neuropsychiatric disorder was made using Diagnostic and Statistical Manual of Mental Disorder V (DSM - V). All data obtained was analysed using statistical package for social sciences Vs 22(SPSS Vs 22). The results were presented as tables and frequencies, significance and correlations were assed using appropriate parameter. $P$ value less 0.5 was considered significant.

Results: A total of 152 patients were recruited for the study, $63.8 \%$ males with mean age of $51.4 \pm 15.5$ years. Majority of them were Ibos (96.7\%), Christian (96.7\%), civil servants (38.2\%) and had tertiary education (46.7\%). Hypertensive nephrosclerosis, diabetic nephropathy and chronic glomerulonephritis were occurred in $40.8 \%, 25 \%$ and $12.5 \%$ of the patients. $55.9 \%$ of them were on haemodialysis however only $12.9 \%$ were regular. Mean PCV, urea and creatinine were $27.8 \pm 12.7 \%, 21.2 \pm 15.1 \mathrm{mmol} / \mathrm{l}$ and $567 \pm 445.8 \mathrm{umol} / \mathrm{l}$.

Depression was seen in $57.9 \%$, dysthymia in $17.8 \%$, panic attack in $10.5 \%$, PTSD in $13.3 \%$, generalized anxiety disorder in $9.7 \%, 6.6 \%$, phobia in $4.6 \%$ and suicidal behavior in $3.9 \%$

Conclusion: Neuropsychiatric manifestation is common in chronic kidney disease patients in south eastern Nigeria.

\title{
Abstract 31:
}

\section{KIDNEY FUNCTION IN FREQUENT USERS OF NON-STEROIDAL ANTI-INFLAMMATORY DRUGS (NSAIDS)}

Uduagbamen $\mathrm{PK}^{1}$, Salako $\mathrm{BL}^{2}$, Hamzat $\mathrm{MA}^{1}$.

${ }^{1}$ Department of Internal Medicine, Federal Medical Centre, Abeokuta; ${ }^{2}$ Department of Internal Medicine, University College Hospital, Ibadan.

Background: Non-steroidal anti-inflammatory drugs (NSAIDs) are analgesic agents commonly used for managing painful conditions. They are readily available over-the-counter drugs, cheap, often taken without prescription and commonly abused. NSAIDs inhibit prostaglandins actions on the kidneys and can cause kidney disease and hypertension, especially when taken in excess doses, prolonged period or in stressed states like dehydration, exercise and heart failure.

Methods: One hundred each of frequent users of NSAIDs (daily use $\geq 4$ weeks) and 100 age and sex matched controls with no known risk for kidney disease and had consented to the study were evaluated thus: history, examination and investigations (urinalysis, serum biochemistry, kidney ultrasound scan and biopsy). Cases with eGFR < $60 \mathrm{mls} / \mathrm{min} / 1.73 \mathrm{~m}^{2}$ ) and dip strip proteinuria $\geq 1+$ had kidney biopsy performed on them. Statistical analysis was with software SPSS 21. Student t-test and Chi square tests were used to compare means and proportions respectively. Pearson's correlation test was used to determine the strength of association between independent risk factors and kidney dysfunction.

Results: Fifty-one (51) females each and 49 (49) males each were recruited as cases and controls respectively. The mean age of the cases and controls were $46.5 \mathrm{yrs} \pm 14.2$ and $46.0 \mathrm{yrs} \pm 14.5$ respectively. The mean age of the cases with KD (63.04yrs \pm 4.21$)$ was statistically higher than those without KD (41.84yrs \pm 14.52$),(P=0.01)$. Majority of the cases were in the working population (30-59 yrs). Twenty-two (22) frequent NSAIDs users were found to have kidney dysfunction (KD) giving a prevalence of $22 \%$ while 6 (6\%) controls group had KD (using CKD-EPI formula). The proportion of subjects that used herbal medicines was higher in cases with KD than in cases without KD and in the controls respectively $(P=0.01)$. The mean length and cortical thickness of the kidneys were statistically significantly lower in cases with $K D$ than in cases without $K D(P=0.03)$ and $(P=0.017)$. The independent predictors of $K D$ were found to be increasing age, use of herbal remedies and duration of drug use. 
Conclusion: This study found a prevalence of $22 \%$ amongst frequent NSAIDs users which is higher than that of the general population. Risk factors observed in this study included increasing age, use of herbal medicines, increasing BMI, systolic BP, anaemia, reduced kidney cortical thickness and kidney volume. The use of NSAIDs in excess doses, prolonged period or in stressed state increases their risk of causing kidney dysfunction, care is therefore needed to avoid taking these drugs in these conditions.

\section{Abstract 32: \\ DIAGNOSIS OF HIVAN: ARE THERE PREDICTORS TO DISTINGUISH HIVAN FROM NON- HIVAN CHRONIC KIDNEY DISEASE?}

Onu Ugochi C, Okoye Julius U, Onu Iheanyi, Onodugo Obinna D, Arodiwe Ejikeme B Chinwuba Ijoma, Dwomoa Adu, Akinlolu Ojo, Babatunde Salako, Desley Neil, Ifeoma Ulasi and the H3AKDRN

Renal Unit, Department of Medicine, University of Nigeria Teaching Hospital, Enugu State, Nigeria.

Background: HIV-Associated Nephropathy (HIVAN) is documented as the most common cause of chronic kidney disease (CKD) in HIV-infected patients but with recent declining prevalence. Although 40-55\% of patients with clinical suspicion of HIVAN have other lesions at biopsy, kidney biopsy however is not easily accessed in developing countries. Aim: To identify more accessible ways to predict HIVAN in HIV-infected patients.

Methods: Seventy-two adult HIV-infected patients with $\geq$ stage 3 CKD underwent real time ultrasound-guided kidney biopsy from April 2016 to August 2018.Fifty-eight samples were adequate for histological diagnosis after evaluation with light microscopy and immunohistochemistry. Excluded were patients having hypertension $>2$ years, diabetes mellitus, other viral infections, obstructive uropathies and malignancies.

Results: Histology was categorized into 13 (22.4\%) HIVAN and 45 (77.6\%) non-HIVAN histology. Five of 16 (31.3\%) patients with CD4 $>500$ cells $/ \mathrm{mm}^{3}$ had HIVAN whereas $9 / 58$ patients with CD4 $<200$ cells $/ \mathrm{mm}^{3}$ had non-HIVAN histology. Other features compared are shown in Tables 1 and 2.

Table1: CATEGORICAL VARIABLE COMPARISON

\begin{tabular}{lllll}
\hline VARIABLE & NON-HIVAN $(n=45)$ & HIVAN $(n=13)$ & $\chi^{2}$ & - value \\
\hline SEX: male & 19 & 6 & 0.064 & 0.801 \\
$\quad$ female & 26 & 7 & & $<0.001^{\times x}$ \\
SIGNS & 31 & 1 & 16.934 & 0.005 \\
None & 14 & 12 & 7.540 & 0.002 \\
Present & 27 & 13 & & \\
Dipstick Proteinuria $>+1$ & & & 11.123 & \\
WHO clinical stage & 38 & 5 & & \\
asymptomatic & 7 & 8 & & \\
symptomatic & & & & \\
\hline
\end{tabular}

$\mathrm{xx}=$ Fisher's exact test, $\mathrm{WHO}=$ World health organization

Table2: CONTINIOUS VARIABLES COMPARISON

\begin{tabular}{lllll}
\hline VARIABLE & NON-HIVAN $(\mathrm{n}=45)$ & HIVAN $(\mathrm{n}=13)$ & $\mathrm{t}$ & $\mathrm{p}$-value \\
\hline Mean age $($ years $) \pm S D$ & $45.24 \pm 9.73$ & $39.08 \pm 8.92$ & 2.049 & 0.045 \\
Mean BMI $\left(\mathrm{kg} / \mathrm{m}^{2}\right) \pm$ SD & $24.78 \pm 2.88$ & $26.54 \pm S D 3.69$ & -1.816 & 0.075 \\
Mean systolic blood pressure $(\mathrm{mmHg}) \pm S D$ & $126.89 \pm 19.98$ & $116.92 \pm 16.52$ & 1.641 & 0.106 \\
Mean Diastolic blood pressure $(\mathrm{mmHg}) \pm S D$ & $76.44 \pm 13.68$ & $71.54 \pm 10.68$ & 1.190 & 0.239 \\
Mean serum creatinine $(\mu \mathrm{mol} / \mathrm{I}) \pm S D$ & $212.21 \pm 104.02$ & $272.31 \pm 140.02$ & -1.693 & 0.096 \\
Mean CD4 cells $/ \mathrm{mm}^{3} \pm S D$ & $350.78 \pm 202.11$ & $299.61 \pm 162.13$ & 0.837 & 0.406 \\
\hline
\end{tabular}

$\mathrm{SD}=$ standard deviation, $\mathrm{BMI}=$ Body Mass index

Conclusion: HIVAN was associated with presence of clinical signs, younger age and higher degree of proteinuria. However larger studies are needed to confirm this and to identify other HIVAN predictors. 


\section{Abstract 33:}

\section{THE PATTERN OF RENAL DISEASE IN HAART-NAÏVE COMPARED TO HAART-EXPERIENCED HIV-INFECTED CKD PATIENTS}

Onu Ugochi C, Okoye Julius U, Onu Iheanyi, Onodugo Obinna D, Arodiwe Ejikeme B Chinwuba ljoma, Dwomoa Adu, Akinlolu Ojo, Babatunde Salako, Desley Neil, Ifeoma Ulasi and the H3AKDRN

Renal Unit, Department of Medicine, University of Nigeria Teaching Hospital, Enugu State, Nigeria.

Background: HIV-Associated Nephropathy (HIVAN) has been documented as the single most common cause of chronic kidney disease (CKD) in HIV-infected patients. However, decline in HIVAN prevalence in populations with access to care has been documented with the widespread use of Highly Active Anti-Retroviral Therapy (HAART).

Aim: The study compared HAART-naïve with HAART-experienced HIV-infected CKD patients to determine if there is any difference in prevalence of HIVAN and also to identify associated factors.

Methods: Seventy-two adult HIV-infected patients with $\geq$ stage 3 CKD underwent real time ultrasound-guided kidney biopsy from April 2016 to August 2018.Fifty-eight samples were adequate for histological diagnosis after evaluation with light microscopy and immunohistochemistry. Excluded were patients having hypertension $>2 y e a r s$, diabetes mellitus, other viral infections, obstructive uropathies and malignancies.

Results: There were 22(37.9\%) HAART-naïve and 36(62.1\%) HAART-experienced patients. Compared variables in table 1 showed HAART-experienced were significantly older with higher mean systolic blood pressure and CD4 cell count. HAART-naïve group had a significantly more advanced WHO clinical and immunological disease ( $p=0.016$ and 0.031$)$.

Table 1: HAART-NAÏVE VERSUS HAART-EXPERIENCED GROUPS COMPARED VARIABLES

\begin{tabular}{lllll}
\hline PARAMETER & HAART-NAÏVE $(\mathrm{n}=22)$ & $\begin{array}{l}\text { HAART-EXPERIENCED } \\
(\mathrm{n}=36)\end{array}$ & $\mathrm{t}$ & $\mathrm{p}$-value \\
\hline Mean age $($ years $)$ & $38.05 \pm 7.00$ & $47.42 \pm 9.67$ & -3.950 & $<0.001$ \\
Weight $(\mathrm{kg})$ & $62.31 \pm 10.60$ & $67.92 \pm 11.34$ & -1.870 & 0.067 \\
Body mass index $\left(\mathrm{kg} / \mathrm{m}^{2}\right)$ & $24.20 \pm 2.69$ & $25.77 \pm 3.26$ & -1.897 & 0.063 \\
Systolic BP $(\mathrm{mmHg})$ & $118.18 \pm 19.18$ & $128.61 \pm 19.00$ & -2.021 & 0.048 \\
Diastolic BP $(\mathrm{mmHg})$ & $72.27 \pm 11.93$ & $77.22 \pm 13.65$ & -1.404 & 0.166 \\
Creatinine $(\mu \mathrm{mol} / \mathrm{l})$ & $241.65 \pm 152.49$ & $215.92 \pm 84.59$ & 0.828 & 0.411 \\
CD4 cell count $\left(\mathrm{mm}^{3}\right)$ & $269.77 \pm 143.73$ & $381.81 \pm 209.35$ & -2.208 & 0.031 \\
Total protein $(\mathrm{g} / \mathrm{l})$ & $76.72 \pm 14.31$ & $79.56 \pm 15.88$ & -0.683 & 0.498 \\
Serum albumin $(\mathrm{g} / \mathrm{l})$ & $35.45 \pm 6.34$ & $35.69 \pm 6.75$ & -0.134 & 0.894 \\
\hline
\end{tabular}

Histology trend is shown in table2 with FSGS (NOS) and HIVAN being the predominant histology in the HAART-

experienced and naïve groups respectively.

Table 2: HISTOLOGY TREND IN HAART NAÏVE VERSUS HAART EXPERIENCED GROUPS

\begin{tabular}{llll}
\hline HISTOLOGY & HAART NAÏVE GROUP & \multicolumn{2}{l}{ HAART EXPERIENCED } \\
GROUP & $\begin{array}{l}\text { TOTAL NUMBER OF EACH } \\
\text { HISTOLOGY }\end{array}$ \\
\hline Minimal chronic damage & $1(4.5 \%)$ & $3(8.3 \%)$ & 4 \\
Arterionephrosclerosis & $0(0.0 \%)$ & $4(11.1 \%)$ & 4 \\
FSGS(NOS) & $4(18.2 \%)$ & $8(22.2 \%)$ & 12 \\
NORMAL & $3(13.6 \%)$ & $5(13.9 \%)$ & 8 \\
HIVAN & $6(27.3 \%)$ & $7(19.4 \%)$ & 13 \\
FSGS (cellular) & $1(4.5 \%)$ & $2(5.6 \%)$ & 3 \\
Chronic interstitial nephritis & $4(18.2 \%)$ & $2(5.6 \%)$ & 6 \\
Focal global glomerulosclerosis & $2(9.1 \%)$ & $5(13.9 \%)$ & 7 \\
BK-virus associated nephropathy & $1(4.5 \%)$ & $0(0.0 \%)$ & 1 \\
Total & $22(100 \%)$ & $36(100 \%)$ & 58 \\
\hline
\end{tabular}

Conclusion: HAART naïve group were younger, had worse disease and were more likely to have HIVAN as the predominant histology.

\section{Abstract 34:}




\section{NEPHROTIC SYNDROME PRESENTING WITHOUT OEDEMA - A CASE REPORT}

Osakwe Nwamaka ${ }^{1}$, Eze Hyacinth ${ }^{1}$, Oguejiofor Fidelis ${ }^{1}$, Ozuemba Bartholomew ${ }^{1}$, Anyanor Sylvester ${ }^{1}$, Ndulue Chidozie ${ }^{1}$, Jisieike-Onuigbo Nonyelum ${ }^{1}$, Kalu Kalu ${ }^{1}$, Odenigbo Charles ${ }^{1}$, Ulasi Ifeoma ${ }^{2}$.

1. Nephrology Unit, Department of Internal Medicine, Nnamdi Azikiwe University Teaching Hospital Nnewi, Anambra State, Nigeria; Department of Internal Medicine, University of Nigeria Teaching Hospital Ituku Ozalla, Enugu State, Nigeria.

Introduction and objective: Nephrotic syndrome is a disorder characterized by proteinuria greater than 3.5 grams per 24hour, hypoalbuminemia less than 30grams per liter, hyperlipidemia and peripheral oedema. Some cases of nephrotic syndrome do not present with oedema, notably secondary nephrotic syndrome like HIVAN. Our objective is to present a case of nephrotic syndrome from focal segmental glomerulosclerosis without oedema at initial presentation.

Methods: A 47year old trader presented at the clinic with complaints of frequency and nocturia. The patient was not a known diabetic and denied use of heroin. On examination he was not obese and had no leg oedema. Urinalysis revealed proteinuria +++ and glucosuria ++. Serum albumin showed hypoalbuminemia. Screening for diabetes, HIV, hepatitis $B$ and $C$ were negative. Serum creatinine was deranged with eGFR of $7 \mathrm{ml} / \mathrm{min} / 1.73 \mathrm{~m}^{2}$. The patient was managed conservatively, and renal function improved but was observed to have new onset leg oedema and persistent proteinuria. He subsequently had a renal biopsy.

Results:

\begin{tabular}{|l|l|l|l|l|}
\hline & Initial & Prebiopsy & Range & Units \\
\hline Creatinine & 925 & 177 & $57-113$ & $\mu \mathrm{mol} / \mathrm{L}$ \\
\hline eGFR & 7 & 46 & & $\mathrm{ml} / \mathrm{min} / 1.73 \mathrm{~m}^{2}$ \\
\hline Urine ACR & 385 & 969 & $<30$ & $\mathrm{mg} / \mathrm{g}$ \\
\hline Urine PCR & & 204 & $<15$ & $\mathrm{mg} / \mathrm{mmol}$ \\
\hline
\end{tabular}

Kidney biopsy showed focal segmental glomerulosclerosis, moderate interstitial fibrosis and mild arteriosclerosis. Conclusion: Oedema may be absent in nephrotic syndrome and a biopsy is important in the evaluation of these patients. In a resource poor setting it may be difficult to exclude potential secondary causes of nephrotic syndrome.

\section{Abstract 35:}

\section{KNOWLEDGE AND PRACTICES OF NURSES REGARDING INPATIENT MANAGEMENT OF CHRONIC KIDNEY DISEASE AT SELECTED REFERRAL HOSPITAL: A NON-EXPERIMENTAL DESCRIPTIVE CORRELATION STUDY}

Emmanuel Gapira B ${ }^{1}$, Geldine Chironda ${ }^{1,2}$, Didace Ndahayo ${ }^{1}$, Michel Nkuranyabahizi ${ }^{1}$, Theos Mbambazi ${ }^{1}$, Jeanne Tuyisengwe $^{1}$, Lakshmi Rajeswaran ${ }^{1,2}$

1. University of Rwanda, College of Medicine and Health Sciences, School of Nursing and Midwifery, Kigali, Rwanda; 2. New York University, Rory Meyers College of Nursing, New York, USA, Human Resources for Health (HRH) Rwanda.

Introduction: Chronic Kidney Disease (CKD) is a serious condition to manage and it requires multidisciplinary team involvement. Nurse's knowledge and practice regarding management of CKD is paramount as this decrease the morbidity and mortality among the population.

Aim: To assess knowledge and practices of nurses regarding inpatient management of CKD at selected referral hospital in Rwanda.

Methodology: This study used a non-experimental descriptive correlation design involving 120 nurses working in internal medicine, emergency and renal units. Data was collected using a self-administered questionnaire. Descriptive statistics, Chi-square, Pearson's correlation coefficient and linear regression were used to analyze the data.

Results: The majority (84\%) had moderate level of knowledge and almost half (51\%) of nurses had moderate level of practice (score 50-79\%) regarding of inpatient management of CKD. The factors associated with knowledge were hospital of practice $(16.3 ; 95 \% \mathrm{Cl} 15.5-17 ; \mathrm{p}=.024)$, work service $(17.6 ; 95 \% \mathrm{Cl} 16.6-18.6 ; \mathrm{p}=.000)$, level of training $(17.4 ; 95 \% \mathrm{Cl} 12.1-22.6 ; p=.010)$ and type of specialty (mean $=19.4 ; 95 \% \mathrm{Cl} 18.4-20.3 ; \mathrm{p}=.000)$. The factor associated with practice was work service (mean $=3.8 ; 95 \% \mathrm{Cl} 3.4-4.3 ; \mathrm{p}=.015$ ). A very weak positive relationship, which nonsignificance between knowledge and practice of inpatient management of chronic kidney disease $(r=.115, N=120, p$ $=.21$ ) have been observed.

Recommendations: In-service training for registered nurses working in the internal medicine and emergency and Further research to elicit the major contributory factors to poor practice should be done. 


\title{
Abstract 36: \\ NON-DIABETIC NEPHROPATHY AMONG DIABETIC PATIENTS OF THE NEPHROLOGY DEPARTMENT OF DAKAR
}

DIAWARA MS, CISSE MM, KEITA N, KANE Y, Mahmoud S, LEMRABOTT AT, FAYE MA, DIAGNE S, KA F, NIANG A, DIOUF B

Introduction: Diabetic nephropathy is the most common cause of kidney disease in diabetics. However, in some cases the clinical symptoms is not typical and nephropathy may be different from diabetic and require the use of renal biopsy which is not usually indicated unless non-diabetic nephropathy is suspected. This study objective's was to evaluate the prevalence of non-diabetic nephropathy among the diabetic patients and to analyse the different predictive factors of its occurrence.

Patients and methods: It was a retrospective, descriptive and analytical study which is carried out at the nephrology department of Aristide Le DANTEC hospital of Dakar over a period of 60 months. Diabetics with suspected nondiabetic nephropathy diagnosis based on renal anomalie that is associated with a recent diabetes, acute renal failure with rapid progress, diabetic retinopathy's absence, extrarenal signs associated with an acute renal failure and microscopic haematuria was included.

Results: Out of 34 biopsied diabetic patients, 12 had non-diabetic nephropathy that is a prevalence of 35, 3\%. The average age was $49.88 \pm 4.15$ years and the mean duration of diabetes is $12.53 \pm 4.7$ years. Glomerular syndrome was found in 30 patients, vascular nephropathy syndrome in 3 patients and tubule-interstitial nephropathy syndrome in only one patient. Diabetic retinopathy and microscopic haematuria respectively existed in 10 patients (34\%) and 15 patients (44. 12\%). The Kidney biopsy show 22 patients (64.7\%) had diabetic nephropathy and 12 patients (38.2\%) presented a non-diabetic nephropathy. Predictive factors of non-diabetic nephropathy diagnosis were a shorter diabetes duration $(P=0.0008)$, high blood pressure $(P=0.0015)$ and absence of diabetic retinopathy $(P=0.005)$. Conclusion: Our data show that kidney injury in a diabetic is not always diabetic nephropathy. The kidney biopsy is often needed in order to adopt an effective management.

\section{Abstract 37:}

\section{HYDRO-ELECTROLYTIC AND ACID-BASE DISORDERS IN NON-DIALYSED CHRONIC KIDNEY DISEASE}

\author{
Cisse MM, Diawara Ms, Kane Y, Lemrabott At, Faye Ma, Fall K, Diallo P, Diagne S, Keita N, Ka F, Niang A, Diouf B
}

Introduction: In Senegal studies on fluid, electrolytes and acid base disorders in non-dialyzed CKD are scarce. The objective of this study was to determine the epidemiological, clinico-paraclinical, etiological and therapeutic aspects with regard to fluid electrolytes and acid-base disorders in non-dialysed patients with chronic kidney disease. Methods: This was a descriptive, analytical and retrospective study performed at the Nephrology Department of LEDANTEC University Teaching Hospital in Dakar from January to December 2017. We included any patient with nondialyzed chronic kidney disease with electrolyte and acid-base results.

Results: Out of the 1023 patients received, 264 were included, making a prevalence of $25.8 \%$. The mean-age of the patients was $52.1 \pm 16.18$ years. Lower limb oedema (36.8\%), dehydration (9.5\%) and high blood pressure (76.8\%) were the main clinical signs found. More than half of the patients had end stage chronic kidney disease $(65,5 \%)$. Hyperphosphatemia was the most common disorder; found in 181 patients or $68.8 \%$, followed by hyponatremia in 96 patients or $36.4 \%$. Hyperkalaemia and hypocalcemia were present in 84 patients $(31.9 \%)$ and 51 patients $(23.4 \%)$ respectively. Metabolic acidosis was found in 4 patients or $1.5 \%$. Forty-four patients or $16.7 \%$ were on sodium polystyrene sulfonate; $31.8 \%$ under calcium carbonate. Forty-five patients (17\%) had a combination of furosemide and fluid restriction, 13 patients (4.9\%) were on normal saline and 3 others (1.1\%) on sodium bicarbonate. Progression was marked by persistence of hyperphosphatemia in $9.8 \%$ of patients, hyponatremia in $5.3 \%$, hypocalcemia in $4.9 \%$ and hyperkalemia in $3.8 \%$. Three patients $(1.13 \%)$ died from pulmonary complications.

Conclusions: Fluid, electrolytes and acid-base disorders are recurrent in advanced stages of chronic kidney disease. Early and more rigorous treatment would prevent particularly acute complications that may be life-threatening. 


\section{Abstract 38: \\ CHRONIC RENAL FAILURE ACUTIZATION: ABOUT A CASE AFTER TAKING CASSIA ITALICA (LAYDOUR) AT THE ZIGUINCHOR PEACE HOSPITAL}

KANE Yaya ${ }^{1 *}$, THIOUBOU ${ }^{1}$, DIALLO K1, KOUTONIN A. Ne ${ }^{1}$, LEMRABOTT AT ${ }^{2}$, FAYE M $^{2}$, FAYE Mo1, DIALLO B1, KEBE A1, SEMBENE L1, CISSE M M ${ }^{4}$, SECK S M ${ }^{3}$, KA EI F², NIANG $A^{2}$, DIOUF $B^{2}$

${ }^{1}$ Department of Nephrology Hemodialysis-Gastroenterology-Internal Medicine Peace Hospital University Assane Seck ZIGUINCHOR; ${ }^{2}$ Nephrology hemodialysis service HALD UCAD Dakar; ${ }^{3}$ Hemodialysis Nephrology Service CHR of St Louis Gaston Berger University; ${ }^{4}$ Thiès hemodialysis nephrology service / Thiès University

Introduction: Acute renal failure induced by medicinal plants in Africa is uncertain because the majority of cases are unreported. We present an observation of acutization of chronic renal failure (CKD) following the consumption of Cassia Italica leaves in a patient followed at the nephrology department of the Ziguinchor Peace Hospital. Clinical observation: Mrs. K.G, 68 years old followed in nephrology department for stable moderate chronic renal failure whose initial nephropathy is benign nephroangiosclerosis. Basic renal function (serum creatinine at $85 \mathrm{mg} / \mathrm{I}$ and blood urea at 0.92 or DFG at MDRD at $48 \mathrm{ml} / \mathrm{min} / 1.73 \mathrm{~m} 2$ ). She consults for uncontrollable vomiting, epigastralgia, obnubilation. Clinical examination revealed $180 / 100 \mathrm{~mm} \mathrm{Hg}$ hypertension and stage II coma with kussmaul dyspnea, anuria, lower limb edema and normal temperature. The biological assessment revealed an impairment of renal function with serum creatinine at $228.5 \mathrm{mg} / \mathrm{l}$ and blood urea at $2.65 \mathrm{~g} / \mathrm{l}$, hemoglobin at $8.1 \mathrm{~g} /$ $\mathrm{dl}$, hypocalcemia at $82 \mathrm{mg} / \mathrm{I}$. The proteinuria of $24 \mathrm{~h}$ has returned to $0.86 \mathrm{~g} / 24 \mathrm{~h}$. The liver test is normal. The blood ionogram shows a hyperkalemia at 6,6 mmol / I. Kidneys' ultrasonography shows poorly differentiated atrophic kidneys. Upper gastrointestinal fibroscopy reveals multiple ulcerations measuring 2 to $4 \mathrm{~mm}$ in the prepyloric antrum probably related to toxicity. The family admits taking the poison 48 hours before hospitalization by excessive consumption of leaves of Cassia italica in the form of a decoction to treat constipation. The diagnosis of acutization of chronic renal failure by acute tubular necrosis of toxic origin was retained. The evolution was favorable, with a resumption of diuresis, disappearance of the symptoms, after a medical treatment and 4 successive hemodialysis sessions in the first week. After 3 weeks of treatment, there is a return to basic renal function (creatinine at $90 \mathrm{mg} / \mathrm{l}$, blood urea at $0.95 \mathrm{~g} / \mathrm{l}$, ie a GFR at $52 \mathrm{ml} / \mathrm{min} / 1.73 \mathrm{~m} 2$ ).

Conclusion: The use of plants by patients treated in nephrology may aggravate pre-existing renal disease. Hence the interest of setting up a phytovigilance system.

\section{Abstract 39:}

\section{KNOWLEDGE AND PRACTICAL ATTITUDES OF STUDENTS AT THE END OF THE MEDICAL STUDY CYCLE ON SCREENING} FOR CHRONIC KIDNEY DISEASE.

Faye Moustapha (1), Keita Niakhaleen (1), Ba Bacary (1), Seck Sidy Mohamed (2), Lemrabott Ahmed Tall (1), Faye Maria (1), Mbengue Mansour (1), Diagne Seynabou (1), Dieng Ahmed (1), Ba Mamadou Aw (1), Diouf Boucar (1), Ka Elhadji Fary (1).

${ }^{1}$ Department of Nephrology, Dialysis and Renal Transplantation of Aristide Le Dantec University Hospital, Cheikh Anta Diop University, Dakar, Senegal; ${ }^{2}$ Department of nephrology and dialysis of the Military Hospital of Ouakam, Gaston Berger University of Saint Louis, Senegal.

Objectives: The aim of this work was to explore the practical knowledge, attitudes and practices and the difficulties of the students at the end of the medical study cycle and the general practitioners (GPs) in the screening of the CKD. Methods: We conducted a descriptive cross-sectional study between January 17 and February 17, 2018 using a questionnaire pre-established by Google forms whose link was shared on social networks. Medical students who validated 5 years of study or GPs in Senegal and agreeing to participate were included.

Results: Sixty-nine respondents ( $27.13 \pm 1.7$ years old, $60.9 \%$ men) were selected for the analysis. They were in doctoral year in $58 \%$, in 7 th year of medicine in $30.4 \%$, GPs in $5.8 \%$. Among the main causes of the CKD, diabetes and hypertension were cited together by $52.5 \%$ of respondents. 
Table1: summary of answers to questions about MRC screening

\begin{tabular}{|c|c|c|}
\hline Questions & Right (\%) & Wrong (\%) \\
\hline \multicolumn{3}{|l|}{ - Do you think that a general practitioner must perform } \\
\hline screening for the MRC? & 95.7 & 04.3 \\
\hline - What tests can he use? & 05.8 & 94.2 \\
\hline - At Moustapha 75 years old, obese with BMI at $34 \mathrm{~kg} / \mathrm{m}^{2}$, & & \\
\hline would you do the screening? & 84.1 & 14.5 \\
\hline $\begin{array}{l}\text { - M. Green, } 28 \text {-year-old asymptomatic with the notion of } \\
\text { edema of the lower limbs at } 7 \text { years old, treated traditionally, } \\
\text { would you do the screening? }\end{array}$ & 07.2 & 92.8 \\
\hline
\end{tabular}

\title{
Abstract 40: \\ SENEGALESE RENAL BIOPSY REGISTRY: DESCRIPTIVE ANALYSIS OF 1559 RENAL BIOPSIES OVER A SEVEN-YEAR PERIOD
}

Lemrabott $A T^{1}$, Faye $M^{1}$, Dial $M^{2}$, Faye $\mathrm{Ms}^{1}$, Cissé $\mathrm{MM}^{3}$, Seck $\mathrm{SM}^{4}$, Kane $\mathrm{Y}^{5}$, Fall $\mathrm{K}^{1}$, Mbengue $\mathrm{M}^{1}, \mathrm{Keita}^{1}, \mathrm{Ba}^{1}$, Diagne $S^{1}$, Dieng $A^{1}, B a A A^{1}$, Niang $A^{1}, K a E H F^{1}$, Diouf $B^{1}$.

${ }^{1}$ Nephrology Department, Aristide Le Dantec University Hospital, Cheikh Anta Diop University, Dakar (Senegal); ${ }^{2}$ Pathology laboratory, Cheikh Anta Diop University, Dakar (Senegal); ${ }^{3}$ Faculty of Medicine. Thies University, Thiès (Senegal); ${ }^{4}$ Faculty of Medicine. Gaston Berger University, Saint-Louis (Senegal); ${ }^{5}$ Faculty of Medicine. Assane Seck University, Ziguinchor (Senegal).

Introduction: Renal biopsy (RB) is performed in few countries in sub-Saharan Africa. However, in our country, it has been practiced for a very long time with an anatomopathological reading done on site since 2009. The purpose of this study was to determine the indications of the RB, and to describe the anatomopathological and etiological aspects of biopsied nephropathies

Methods: This is a retrospective descriptive study over a 7 years period (1st January 2010 to 31st December 2016). We included patients had RB during the study period with cortical histological section and containing at least 10 glomeruli. All biopsies were ultrasound guided.

Results: 1559 PBR were realized. The mean age of the patients was $33.8 \pm 16.6$ years. There were 834 men for 725 women, a sex ratio of 1.15 . Nephrotic syndrome was the main indication in $78.3 \%$ of cases. Glomerular lesions accounted for $58.82 \%$ of cases, tubulointerstitial lesions $3.91 \%$ of cases, vascular lesions $7.11 \%$ of cases, and lesions unclassified or uninterpretable $29.05 \%$. Among the glomerular lesions, focal segmental glomerulosclerosis (FSGS) accounted for $27.48 \%$ of cases, membranous nephropathy (MN) $10.68 \%$ of cases, minimal change disease (MCD) $29.00 \%$ of cases, membranoproliferative glomerulonephritis (MPGN) $1.85 \%$, extracapillary glomerulonephritis (EGN) 4.79\%, endocapillary glomerulonephritis $3.05 \%$, IgA nephropathy $2.18 \%$ and lupus nephritis accounted for $13.74 \%$. Conclusion: PBR plays a central role in the nosological, diagnostic and therapeutic approach in nephrological practice. In our context glomerular lesions are dominated by MCD and FSGS. The latter is partly due to a genetic predisposition linked to a polymorphism and to the existence of two variants (G1 and G2) of the gene coding for APOL1.

\section{Abstract 41:}

\section{EPIDEMIOLOGICAL, BIOLOGICAL, THERAPEUTIC AND EVOLUTIONARY PROFILE OF PROLIFERATIVE LUPUS NEPHRITIS: A REPORT ABOUT 44 CASES IN DAKAR}

\author{
Mbengue $\mathrm{M}^{1}$, Faye $\mathrm{M}^{1}$, Lemrabott $A T^{1}$, Cissé $\mathrm{MM}^{2}$, Dial $\mathrm{MC}^{3}$, Seck $\mathrm{SM}^{4}$, Kane $\mathrm{Y}^{5}$, Fall $\mathrm{K}^{1}$, Faye $\mathrm{Ms}^{1}$, Ba $\mathrm{B}^{1}$, Diagne $\mathrm{S}^{1}$, \\ Keita $\mathrm{N}^{1}$, Dieng $\mathrm{A}^{1}$, Ba $\mathrm{M} \mathrm{A}^{1}$, Niang $\mathrm{A}^{1}$, Ka EHF ${ }^{1}$, Diouf $\mathrm{B}^{1}$. \\ ${ }^{1}$ Nephrology Department, Aristide Le Dantec University Hospital, Cheikh Anta diop University, Dakar (Senegal); ${ }^{2}$ Faculty \\ of Medicine. Thies University, Thiès (Senegal); ${ }^{3}$ Pathological laboratory, Cheikh Anta diop University, Dakar (Senegal); \\ ${ }^{4}$ Faculty of Medicine. Gaston Berger University, Saint-Louis (Senegal); Faculty of Medicine. Assane Seck University, \\ Ziguinchor (Senegal).
}

Introduction: Proliferative lupus nephritis is the most common type of lupus nephritis. They engage the renal prognosis. The aim of this study is to describe the epidemiological, clinical, biological, therapeutic and evolutionary profile of proliferative lupus nephritis. 
Methods: This is a retrospective descriptive study conducted over a 10-year period from January 1, 2007 to December 31, 2016 in the Nephrology Department of Aristide Le Dantec University Hospital. Patients followed for proliferative lupus nephritis were included.

Results: Of 64 patients, 44 patients had proliferative lupus nephritis (68.75\%). The mean age age was 31.81 years \pm 11.27. The sex-ratio was 0.33 . Renal failure was present in admission in 20 patients (45.5\%). The average proteinuria was $3.97 \pm 2.7 \mathrm{~g} / 24 \mathrm{~h}$. Class III was found in $11.3 \%$ of cases, class IV in $27.3 \%$, class III $+\mathrm{V}$ in $43.2 \%$, and class IV $+\mathrm{V}$ in 18 , $2 \%$ of cases. In induction therapy, cyclophosphamide was used in 22 patients, mycophenolate mofetil in 10 patients and azathioprine in 3 patients. Immunosuppressive therapy was associated with methylprednisolone boluses daily for 3 doses followed by prednisone per os. In maintenance therapy, azathioprine was used in all patients. Of the 44 patients, 35 were followed for 6 months. Of these 35 patients, $22.8 \%$ were in complete remission, $20 \%$ in partial remission and $57.14 \%$ were resistant. Death was noted in 5 patients. Chronic renal failure occurred in 8 cases. Treatment-related complications were infectious in 17 patients and cutaneous (32\%) and pulmonary (16\%) localizations were the most common.

Conclusion: The prognosis of proliferative lupus nephritis is pejorative in our practice setting. The risk of chronic renal failure is relatively high because of a high rate of resistance to treatment.

\title{
Abstract 42:
}

\section{AUTOSOMAL DOMINANT POLYCYSTIC KIDNEY DISEASE: EXPERIENCE OF THREE CENTERS IN SENEGAL}

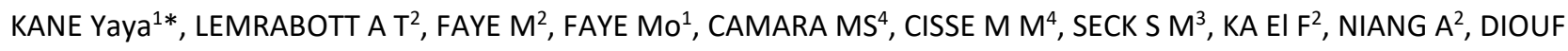 \\ $B^{2}$. \\ ${ }^{1}$ Nephrology service hemodialysis-gastroenterology-internal medicine peace hospital Assane University Seck \\ ZIGUINCHOR; ${ }^{2}$ Haemodialysis Nephrology Department HALD UCAD Dakar; ${ }^{3}$ Hemodialysis Nephrology Service CHR of St \\ Louis Gaston Berger University; ${ }^{4}$ Nephrology service hemodialysis CHR Thiès / University Thiès.
}

Introduction: Autosomal dominant polycystic kidney disease (PKRAD) is one of the most common genetic diseases in the world. The purpose of our study was to describe the profile of patients with PKRAD and to evaluate the search for a gene mutation and the use of new drugs in our patients.

Methods: This was a prospective, multicenter, transversal study over 3 years from $1^{\text {rst }}$ January 2015 to $31^{\text {th }}$ December 2017. Were included in the study, all patients hospitalized or received for consultations, in three nephrology departments (Ziguinchor, Thiès and St. Louis) for PKRAD and over the age of 15 years.

Results: Over the 03 years of study, 25 cases of PKRAD were collected or a prevalence of $0.30 \%$. The average age was 45.56 years old. High blood pressure was present in all our patients. The family history of PKRAD was found in 14 patients (56\%). Large palpable kidneys were found in 22 patients (85\%) followed by lumbar pain in 21 patients (60\%) and macroscopic hematuria was found in 2 cases ( $8 \%)$. The majority of patients had renal failure according to diagnosis. Extra-renal cysts were found mainly in the liver of 8 patients (68\%). The average hemoglobin level was 9.91 $\mathrm{g} / \mathrm{dl}$. Cytobacteriological examination of the urine was positive in 4 patients (16\%) isolating an Escherichia coli in 3 patients and one case of trichomonas vaginalis. A genetic study was performed in 2 patients (8\%) and confirmed a PKD1 gene mutation Hypertension was treated with monotherapy in 5 patients (20\%), dual therapy in 13 patients $(13 \%)$ or triple therapy in 7 patients $(28 \%)$. Four patients $(16 \%)$ had benefited from chronic hemodialysis. Only one patient had received treatment with Vaptan (4\%). The evolution was marked by the occurrence of 4 deaths, ie $16 \%$. Conclusion: PKRAD in black African subjects is not uncommon and probably underdiagnosed. The genetic study provides a better understanding of the large phenotypic variability of PKRAD.

\section{Abstract 43: \\ EPIDEMIOLOGICAL, CLINICAL AND EVOLUTIVE PROFILE OF AUTOSOMAL DOMINANT POLYCYSTIC KIDNEY DISEASE (ADPKD) IN TOGO}

Tsevi Yawovi Mawufemo, Amekoudi Eyram Yoan, Djagadou Kodjo Agbeko, Tona Komlan Georges, Dolaama Badomta, Attisso Eugene, Sabi Kossi Akomola

Objective: To describe the epidemiological, clinical and evolutionary profile of ADPKD in Togo.

Methods: A retrospective descriptive transversal study over a period of 8 years (2011-2018) which focused on the analysis of patients' records diagnosed with ADPKD. The diagnosis of ADPKD was retained on the basis of the ultrasound criteria of PEI. 
Results: During the study period, 27 patients had polycystic kidney disease with a prevalence of $0.87 \%$. The average age was $51.6 \pm 16.4$ years. There were 10 men (37\%) and 17 women (63\%), a sex ratio (M/F) of 0.58 . The concept of family cystic kidney disease was found in 6 (22.2\%) patients. The clinical presentations were dominated by arterial high blood pressure, abdominal pain and abdominal mass respectively in $77 \%, 63 \%$ and $63 \%$ of cases. Five patients $(18.5 \%)$ had a glomerular filtration rate (GFR) greater than $90 \mathrm{ml} / \mathrm{min}, 17(62.9 \%) \mathrm{had}$ a GFR $<60 \mathrm{ml} / \mathrm{min}$. All patients $(100 \%)$ had multiple renal cysts, 16 patients $(59.3 \%)$ had dedifferentiated kidneys. Six patients $(22.2 \%)$ had liver cysts, one patient (3.7\%) had lithiasis. Genetic was not achieved because of the poor technical platform and the high cost of these tests.

Conclusion: ADPKD is common in our department. It appears to be associated with a high rate of chronic renal failure.

\title{
Abstract 44:
}

\section{HIV RELATED KIDNEY DISEASES IN A COUNTRY WITH A LOW HIV PREVALENCE}

\author{
${ }^{1}$ Hanen Abid, ${ }^{1} \mathrm{kkram}$ Agrebi, ${ }^{1}$ Olfa Zini, ${ }^{2} \mathrm{Hana}$ Chaabouni, ${ }^{1}$ Salma Toumi, ${ }^{1}$ Khawla Kammoun, ${ }^{2}$ Chekib Marrekchi, \\ ${ }^{1}$ Soumaya Yaich, ${ }^{3}$ Tahya Boudawara, ${ }^{2}$ Mounir Ben Jemaa, ${ }^{1}$ Mohamed Ben Hmida \\ Departments of ${ }^{1}$ Nephrology, ${ }^{2}$ Infectiology and ${ }^{3}$ Histopathology, Hedi Chaker Hospital, Sfax Tunisia.
}

Introduction: Renal impairment in Human Immunodeficiency Virus (HIV) infected patients is frequent. HIVAssociated Nephropathy (HIVAN) with glomerulosclerosis is the most common form specially in black race. But other glomerular entities were also described.

\section{Case reports:}

Case 1: A 20-year-old woman from sub-Saharan Africa presented in the emergency room with vomiting. Investigations revealed renal failure with serum creatinine of $1183 \mu \mathrm{mol} / \mathrm{I}$ and nephrotic range proteinuria of $32 \mathrm{~g} / 24 \mathrm{~h}$. Renal ultrasound demonstrated an extremely high echogenic cortex.

Serological test for the HIV was positive with low CD4+ cells counts. Screening for malaria was positive. Kidney biopsy showed collapsing focal segmental glomerulosclerosis. Antiretroviral therapy (ART) was started. Malaria was successfully treated. But renal failure persisted requiring maintenance hemodialysis regularly.

Case 2: A 40-year-old man with a history of HIV infection was admitted for nephrotic syndrome. Serum creatinine level was $235 \mu \mathrm{mol} / \mathrm{l}$. Kidney biopsy revealed membranoproliferative glomerulonephritis type 1 . Serological tests for hepatitis $B$ and $C$ were negative. He received ART in addition to standard therapy for kidney disease including ACE inhibitors. After 5 years of follow up, the patient progressed to end stage renal disease (ESRD).

Conclusion: HIVAN is the most common form in HIV-infected patients. It is still the major cause of ESRD in this population. Other histological entities with better prognosis were also described, such as immune mediated glomerulonephritis. Physicians in charge of HIV-infected patients should be aware of the importance of screening for kidney abnormalities because early intervention is essential in slowing progression of kidney disease.

\section{Abstract 44:}

\section{SHUNT NEPHRITIS, A CASE REPORT}

Cyriaque Mbarubukeye1, Bashir Adman²

${ }^{1}$ East Africa Kidney Institute; ${ }^{2}$ Paediatric Department, University of Nairobi and Kenyatta National Hospital.

Background: shunt nephritis is rare acute kidney injury caused by a complication of long standing infected ventriculo peritoneal or ventriculoarterial shunt.

Case report: A 8 years old male infant treated with ventriculoperitoneal shunt for hydrocephalus secondary to craniopharyngioma 1 year ago was admitted to our hospital with 5 days history of hotness of the body, facial swelling, blood urine, and 2 days history of cough and vomiting. At admission he was sick looking, moderate pallor, facial puffiness, febrile, his blood pressure was $131 / 101 \mathrm{mmhg}$, tachypneic with acidotic breathing. Initial lab works revealed elevated CRP at 68.2, proteinuria 3 plus and haematuria $2+$, leucocytosis (18.23), neutrophilia of 13 (85\%)anemia with haemoglobin of $6.4 \mathrm{~g} / \mathrm{dl}$, thrombocytopenia of 87 , hypernatremia of 186 , hyperkalemia of 6.2 , urea of $26 \mathrm{mmol} / \mathrm{L}$, creatinine of $502 \mu \mathrm{mol} / \mathrm{l}$ and estimated GFR of 8.2.blood gas analysis showed high anion gap metabolic acidosis. Cerebrospinal fluid analysis was cloud, lower glucose of $2.2 \mathrm{mmol} / \mathrm{l}$, higher protein of $600 \mathrm{mg} / \mathrm{dl}$, cell count of 9 white blood cell/ $\mathrm{mm} 3$ but there was no growth obtained in culture. He received intravenous fluid, ceftazidime renal adjusted dose, packed red cell transfusion, antihypertensive drugs, and urgent hemodialysis was initiated. The kidney function normalized after 3session of hemodialysis and hemodialysis catheter removed after 7 days. The 
neurosurgeons were consulted regarding of removal of the shunt advised conservatives management because of advanced stage of tumour and the child died at 16 days.

DISCUSSION: Shunt nephritis is a rare disease of the kidneys caused by bacterial antigen and human antibody immune-complex deposition in the glomerulus following the placement of a ventriculoperitoneal (VP) or ventricular atrial (VA) shunt inserted for palliation of congenital or acquired hydrocephalus. Shunt nephritis is seen more commonly in patients with ventriculo atrial shunts compared to those with ventriculo peritoneal shunts.

Staphylococcus epidermidis, a coagulase- negative staphylococcus, is the most common pathogen in shunt nephritis. Conclusion: Treatment of the shunt nephritis has historically been a combination of administration of antibiotics to eliminate the bacterial infection, removal of the infected shunt, and replacement of the infected shunt.

\title{
INTERVENTIONAL
}

\author{
Abstract 45: \\ THE CONSEQUENCES OF NEGLECTING VASCULAR STEAL AMONG HAEMODIALYSIS PATIENTS WITH ARTERIOVENOUS \\ FISTULAE \\ Osman Osman ${ }^{1,4}$, Mohamed A. Marie ${ }^{3,5}$, Tarek Demerdash ${ }^{3,5}$, Nicholas Lagattolla ${ }^{2}$ \\ Al-Hada Military Hospital, Taif, Saudi Arabia, Departments of Plastic ${ }^{1}$, Vascular Surgery ${ }^{2}$ and Nephrology ${ }^{3}$; Al-Azhar \\ University ${ }^{4}$, Cairo, Egypt; Cairo University ${ }^{5}$, Cairo, Egypt.
}

\section{Abstract 46:}

\section{HAEMODIALYSIS VASCULAR ACCESS FUNCTION IN DIALYSIS PATIENTS AT THE KENYATTA NATIONAL HOSPITAL}

\author{
F.O. NDINYA ${ }^{1,2}$, J.K. KAYIMA ${ }^{1,2}$, P.C. MAGABE ${ }^{3}$, S.O. McLIGEYO1,2, A.J.O. WERE ${ }^{1,2}$, G.O ODINYA ${ }^{1,2}$. \\ 1. The University of Nairobi Department of Clinical Medicine and Therapeutics; 2. Kenya Renal Association; 3. \\ Department of Diagnostic Imaging and Radiation Medicine, University of Nairobi.
}

Background: The number of patients requiring renal replacement therapy (RRT) worldwide has been on a rise. Vascular access (VA) is the life-line for the majority of these patients when haemodialysis (HD) is the treatment of choice. VA related morbidity is a leading cause of hospitalization and its function and patency is essential for the optimal management of patients. There is need to determine VA that are dysfunctional since intervention would preserve function as well as preserve future vascular access sites.

Objectives: To evaluate the haemodialysis vascular access function in patients undergoing dialysis at the Kenyatta National Hospital renal unit using mean and peak blood flow rates, urea reduction ratio and Kt/V.

Methods: This was a cross-Sectional descriptive study at Renal unit, Kenyatta National Hospital where one hundred and fifty patients on haemodialysis were analysed.

Results: The non-cuffed non-tunneled central venous catheter (CVC) performed poorly; only $47.5 \%$ achieved a mean blood flow rate above $300 \mathrm{ml} / \mathrm{min}$ as compared to arteriovenous fistula (AVF) $87.5 \%$ and cuffed tunneled CVC (81.7\%). Twenty four percent of patients had a URR less than $65 \%$. A higher mean blood flow rate was associated with a higher URR ( $p=0.004)$ and $\mathrm{Kt} / \mathrm{V}(\mathrm{p}=0.009)$. AVF stenosis was present in $12.5 \%$ and thrombosis in $3 \%$ of patients. Aneurysms were the commonest AVF complication at $46.9 \%$ but were not haemodynamically significant.

Conclusion: Tunneled cuffed haemodialysis CVCs offered adequate blood flows and achieve good delivered dialysis dose that were comparable to arterio-venous fistula. The non-tunneled non-cuffed CVCs delivered poor blood flow rates and dialysis dose and are in use for longer than the recommended duration. Arterio-venous fistula access had better blood flow rates, delivered dialysis dose and were in use for a longer duration. A high mean blood flow rate was associated with a higher delivered dialysis dose. There is a need to have routine surveillance and make interventional procedures affordable to prevent vascular access loss. 


\title{
Abstract 47: \\ COMPLICATION OF PERMANENT HAEMODIALYSIS CATHETER INSERTION: RIGHT INTERNAL JUGULAR PERFORATION
}

Lula Kithome, Anthony Gikonyo, Isaac Adembesa, Benjamin Wambugu, Premanand Ponoth

The Karen Hospital, Nairobi, Kenya.

\section{Abstract 48:}

\section{CORONARY ARTERY BYPASS GRAFT SURGERY IN PATIENTS WITH CHRONIC KIDNEY DISEASE}

\author{
PREMANAND PONOTH, BOB KIKWE, HUSSEIN KHALIF, OBED MORARA
}

Background: During the past one year we have performed over 30 coronary artery bypass graft surgeries (CABG) Objective: To study patient outcome for Chronic Kidney Disease patients undergoing CABG

Methods: Review of patients with respect to risk factors, hospital stay, requirement for haemodylisis and mortality. Results: 30 Patients were operated within the period of April 2017 to April 2018. 20 patients were done off cardiopulmonary bypass, while 10 done on cardiopulmonary bypass. 5 of these patients had Chronic kidney disease. All the patients with kidney disease were done off Cardiopulmonary bypass. 3 required hospital dialysis, prolonged hospital stay and one mortality.

Discussion: Patient with CKD are a sun group of high risk patients undergoing CABG. Significant reduced postoperative recovery time was noted in the off $C P B$ group in regards to weaning off ventilation and overall hospital stay. For the 5 CKD patients who underwent CABG, 2 did not require dialysis and were discharged after 7 days' postoperative, 3 required dialysis and had an overall hospital stay of 14 days. The one mortality occurred 14 days' post operatively.

Conclusion: There is hope in doing CABG in CKD patients with expected favorable outcomes. Doing off CPB, shows more promising results as reduction in perioperative time occurs which improves on post-operative outcome.

\section{Abstract 49:}

COMPLICATIONS OF THE FIRST NATIVE ARTERIOVENOUS FISTULA IN ITS FIRST YEAR: RISK FACTORS, EVOLUTION, TREATMENT (ABOUT 81 CASES)

CISSE MM, DIAWARA MS, KANE Y, EDDIN EJS, LEMRABOTT AT, FAYE Ma, FALL K, DIALLO P, DIAGNE S, KEITA N, KA F, NIANG A, DIOUF B

Introduction: Native arteriovenous fistula (FAV) is the vascular access of choice in chronic haemodialysis because of its durability and lower incidence of complications. Several factors are responsible of its failure.

The goal of our work was to determine the complications associated with AVFs and factors behind its failure in the first year of in its creation in our chronic hemodialysis patients.

Methods: We carried on a multicentric transversal, descriptive and analytical study over a 2-month period from August 1, 2018 to September 30, 2018, in Senegal's various hemodialysis units. We included all chronic hemodialysis patients who started dialysis in different dialysis centres in Senegal with a minimum duration of one year. We analyzed demographic, clinical and biological parameters of our patients and the AVfs' characteristics. In order to identify the complications and failure risk factors of the first native AVF, we divided our patients into 2 groups: group1 (patients having lost their first AVF), group 2 (patients with a functional AVF at the end of the study).

Results: Our study included 452 chronic hemodialysis patients aged $48.06 \pm 14.67$ years, with a slight male predominance (sex ration is 1.13); initial nephropathy is indeterminate in $34 \%$ of cases. Statistical analysis showed a percentage of first native AVF' complication in $17.92 \%$ of cases. The main risk factors were: HTA $(p=0.03)$; AVF radial site $(p=0.01)$; Short puncture time after creation $(p=0.03)$; Early failure $(p=0.012)$; Thrombosis $(p=0.03)$; not taking erythropoietin $(p=0.05)$.

Conclusion: Risk factors are numerous. A surveillance strategy and multidisciplinary management approach are imperative for the long-term perenniality of native AVF. 


\section{Abstract 50: \\ INCIDENCE OF COMPLICATIONS IN ADULTS AFTER PERCUTANEOUS NATIVE RENAL BIOPSY IN LOW TO MIDDLE INCOME COUNTRIES: A SYSTEMATIC REVIEW}

Shepherd Kajawo ${ }^{1,2}$, Jean Jacques Nobiap ${ }^{3}$, Udeme Ekrikpo ${ }^{1,4}$, Andre Pascal Kegne ${ }^{3,5}$, Ikechi Okpechi ${ }^{1}$ 1 - Division of Nephrology and Hypertension, University of Cape Town, South Africa; 2 - Division of Clinical Practice and Patient Care, National University of Science and Technology, Bulawayo, Zimbabwe; 3-Department of Medicine, Groote Schuur Hospital and University of Cape Town, Cape Town, South Africa; 4- Renal Unit, Department of Medicine, University of Uyo, Uyo, Nigeria; 5- Medical Research Council of South Africa, Cape Town, South Africa.

Background: Kidney biopsy is essential in guiding clinicians to diagnose, treat and prognosticate renal disease. However, the procedure can be marred by various complications. The reported occurrence varies among countries or regions and is also affected by several clinical and technical factors. This systematic review and meta-analysis aims to evaluate the incidence of major complications after percutaneous renal biopsy in low to middle income countries (LMIC).

Methods: We included studies of populations from LMIC as per the published World Bank 2017 country list. Relevant abstracts published from 1 January 1980 to 31 December 2017 were searched in PubMed. Two review authors independently screened, selected studies, extracted data and assessed the risk of bias in each study. A third reviewer arbitrated in cases of disagreements. The data was entered on Excel spreadsheet and analysed according to geographical region and Income group

Results: 35 studies with 18447 patients representing 18456 biopsies met inclusion criteria. The ages ranged 3 to 84 years. The least number of studies were from Europe \& Central Asia representing $5.7 \%$. The majority of studies were from middle income (88.6\%) with low income countries representing only $11.4 \%$. Mean ages ranged from 21.0 to 45.5 years. Females ranged from 10.5 to $73.3 \%$. The majority of studies were of moderate quality (80\%) whereas low quality studies were $17.1 \%$ and there was only one (2.9\%) high quality. Major complications were $0.01(0.01-0.02)$.The overall complication rate was $0.14(0.10-0.19)$. On comparing procedures, the blind technique had highest complication rate $0.27(0.19-0.36)$ compared to ultrasound guidance $0.13(0.08-0.19$. There was no death reported in all studies. Nephrectomy was only reported in one middle income country.

Conclusion: There are few studies reported from low income countries. The overall complication rates in LMICs are generally safe. Ultrasound techniques are safest. There is need for intervention to increase biopsy rates in low income countries.

\section{Abstract 51:}

\section{VASCULAR ACCESS FOR HEMODIALYSIS IN TOGO: A MULTICENTER NATIONAL STUDY OF 104 CASES}

DOLAAMA B. ${ }^{1}$, AMEKOUDI E.Y.M. ${ }^{1}$, SABI K.A. ${ }^{1}$, TSEVI Y.M. ${ }^{1}$, PATSOH AMOUZOU K. ${ }^{1}$, TONA K.G. ${ }^{1}$, ATTISSO A.E. ${ }^{1}$, NOTOKADOU-KAZA B. ${ }^{1}$, ADABRA K. ${ }^{2}$

1: Nephrology Service of Sylvanus Olympio Teaching Hospital, Lome, TOGO; 2: Surgery Service of CHU Sylvanus Olympio Teaching Hospital, Lome, TOGO.

Objectives: Evaluate vascular access for hemodialysis in Togo while determining the types of vascular access used in hemodialysis in Togo, their longevity and the factors that may influence the duration or survival of these vascular accesses.

Methods: This was a cross-sectional national prospective descriptive and analytical study with a study population consist of all kidney failure patients in hemodialysis who agreed to participate in the survey and who were followed up during one year (from March 1, 2017 to February 28, 2018) in their various hemodialysis centers.

Results: Among the 104 patients, most came from CHU SO (61.5\%) in Lomé. The average age was 48.2 years \pm 12.6 . The sex-ratio was 1.9. Most of our patients were unemployed (48. 1\%) and with low socioeconomic status (67.3\%). The most common comorbidities were high blood pressure $(94,38 \%)$ and diabetes $(13,46 \%)$. Therefore, vascular nephropathy was predominant in etiologies. We found the predominant use of temporary catheters (34,6\%). Distal arteriovenous fistulas last longer ( 51,6 months on average or 4 years $\pm 48,4$ months) followed by proximal arteriovenous fistulas (47,5 months on average or 3 years 9 months $\pm 41,2)$. According to Kaplan-Meier analysis the high average survival time $(14,1$ months) of the catheters was related to the nephrologist and that high one of the arteriovenous fistulas was related to the surgeons; the high survival time of the proximal arteriovenous fistulas compared to other vascular accesses was statistically significant $(p<0.05)$. 


\title{
Abstract 52: \\ COMPARISON OF ADVANCED IMAGE-GUIDED PERCUTANEOUS (AIP) VERSUS ADVANCED LAPAROSCOPIC SURGICAL (ALS) TECHNIQUE FOR THE PLACEMENT OF PERITONEAL DIALYSIS (PD) CATHETERS
}

\author{
Sijie Zheng; Todd Drasin; Paul Dybbro; Jeanne Darbinian; Neelam Bhalla \\ Kaiser Permanente Northern California.
}

Background: PD offers several advantages to patients with ESRD. The correct and timely placement of a functional PD catheter with minimal complications is crucial to ensure long term PD success. We conducted a retrospective cohort study to compare overall catheter survival, complications, and access time between the techniques.

Methods: The source population was Kaiser Permanente Northern California (KPNC) members who had a PD catheter placed between $1 / 1 / 11$ and $12 / 31 / 2013$ by either the AIP or the ALS technique. Procedure notes were reviewed by two nephrologists and two Intervention Radiologists. Bivariate analyses were conducted to compare descriptive and clinical characteristics, Chi-square tests for categorical variables and t-tests and Wilcoxon two-sample tests for continuous variables. Modified least squares regression was used to estimate adjusted one-year catheter survival. Results: We identified 191 PD catheter placed via AIP and 238 via ALS technique. Adjusted one year PD catheter survival was $80 \%$ by AIP vs. $91 \%$ by ALS. Major complications (bowel perforation, bladder puncture, arterial laceration, and major bleeding) were extremely rare in either group (<1\%). Minor complications (infection, hernia, skin breakdown, bleeding, and pain) were $45.6 \%$ in AIP and 38.7\% in ALS (P: 0.17). Median access time was 12 days in AIP and 33 days in ALS.

Conclusion: Both AIP and ALS techniques have excellent one year survival with very low major complication rates. The slightly lower one year survival rate for the AIP technique is still within the ISPD recommendation. AIP has shorter access time. The techniques are complementary to each other; therefore either should be used based upon local resources and expertise to increase the adoption of PD.

\section{HAEMODIALYSIS}

\section{Abstract 53:}

\section{PROTECTIVE FACTORS OF COGNITIVE DISORDERS IN CHRONIC HEMODIALYSIS FOLLOWED AT THE TEACHING NATIONAL HOSPITAL OF COTONOU (BENIN) IN 2018}

Séraphin AHOUI ${ }^{1}$, Bruno L AGBOTON ${ }^{2}$, Hermann BIAO ${ }^{1}$, Jacques VIGAN ${ }^{2}$, Edouard HOUNYE ${ }^{1}$; Francis T TOGNON ${ }^{1}$, Anselme DJIDONOU ${ }^{1}$; Prosper GANDAHO ${ }^{1}$

${ }^{1}$ Faculty of Medicine, University of Parakou; ${ }^{2}$ Faculty of Health Sciences, University of Abomey -Calavi.

Background: Cognitive disorders in hemodialysis remain multifactorial. Prevention is necessary.

Objective: to study the protective factors related to the absence of cognitive disorders in chronic hemodialysis patients of the Teaching National Hospital (TNH) of Cotonou (Benin) in 2018.

Patients and methods: This was a cross-sectional descriptive and analytic study that took place from March $25^{\text {th }}$ 2018 to July $28^{\text {th }}, 2018$ at TNH of Cotonou. Recruitment was exhaustive. Included were all hemodialysis longer than 3 months, at least 18 years old and consenting. The absence of cognitive impairment was the dependent variable and was assessed using the Senegal Test and the Rapid Frontal Efficiency Battery (RFEB). The data was entered using Epi Data 3.1 en and analyzed using Epi info7 with a significance level of 5\%.

Results: A total of 113 chronic hemodialysis patients were included. The mean age was $49.34 \pm 12.34$ years with a sex ratio of 2.23. Average seniority in hemodialysis was $6.61 \pm 5.02$ years. Of the 113 participants, $73.45 \%$ and $35.79 \%$ had no cognitive impairment in the Senegal Test and the RFEB, respectively. Protective factors in favor of the absence of cognitive disorders were regular consumption of fruits and vegetables $(p=0.04)$ and good quality of extra-renal cleansing $(\mathrm{p}<0.00001)$.

Conclusion: Good prevention of cognitive disorders in hemodialysis patients requires good dialysis and regular consumption of fruits and vegetables while preventing hyperkalemia. 


\section{Abstract 54: \\ MORTALITY IN MAINTENANCE HEMODIALYSIS IN A SUB-DIALYSIS CONTEXT AT THE YALGADO OUEDRAOGO UNIVERSITY HOSPITAL}

Abdoul Hassane Traoré*, Ahmad Nasir Saud, Gaoussou Sanou, Juste Bonzi, Aida Lengani, Ignace Nikièma, Gérard Coulibaly

Department of Nephrology, Yalgado Ouédraogo University Hospital, Ouagadougou, Burkina Faso.

Objectives: This study aimed at analysing mortality among maintenance hemodialysis patients at the Yalgado Ouédraogo University Hospital in Ouagadougou, Burkina Faso.

Methods: This was a retrospective, single-center study that included all patients on maintenance hemodialysis who died between January 1, 2016 and December 31, 2018 in the hemodialysis unit. For various reasons, the frequency of sessions was one every four to five days for one third of patients.

Results: The average annual number of hemodialysis patients was 475 . Three hundred and thirty-seven patients died (113 patients per year on average), resulting in a total annual mortality rate of $23.7 \%$. The sex ratio was 1.5 . The average age of these deceased patients was $45.2 \pm 17.9$ years with extremes of 10 years and 95 years. The average duration of dialysis was $13.3 \pm 25$ months with extremes of 02 weeks and 15 years. Out of the number of patients who died, $\mathbf{8 1 . 3 \%}$ started dialysis in an emergency setting. Severe sepsis was implicated in $43.8 \%$ of deaths; it was linked to an infection of the central venous catheter (CVC) in $76.8 \%$ of cases.

Conclusion: The mortality rate in our hemodialysis unit was high, although comparable to rates reported in some African series with a higher frequency of sessions. Death was usually early and often related to CVC infection. Resolving first and foremost permanent vascular problems would help to significantly reduce the mortality rate.

\section{Abstract 55:}

\section{ASSOCIATION BETWEEN ANXIETY AND DEPRESSION DISORDERS AND HEALTH RELATED QUALITY OF LIFE IN MAINTENANCE HAEMODIALYSIS PATIENTS.}

Alex Mambap $\mathrm{T}^{1}$, Murielle Gaingne $\mathrm{K}^{1}$, Gloria E. Ashuntantang ${ }^{2}$

${ }^{1}$ Faculty of Health Sciences, The University of Bamenda; ${ }^{2}$ Faculty of Medicine and Biomedical Sciences, The University of Yaoundé I.

Objective: The aim of this study was to find the prevalence of anxiety and depression in patients on maintenance haemodialysis and their impact on the quality of life in the Bamenda Regional Hospital.

Methods: This analytical and cross-sectional study was conducted from January to April 2018 in the Bamenda Regional Hospital. Those included were the consent patients who were on MHD. Patients with documented intellectual disabilities were excluded. Subjects completed the Beck Anxiety Inventory (BAI) and Beck Depression Inventory-II (BDI) as well as the Hospital Anxiety and Depression Scale (HADS). The health related quality of life (HRQOL) was assessed using the Kidney Disease Quality of Life-Short Form (KDQoL-SF36).

Results: A total of 65 patients with a mean age of 45.57 ( \pm 15.13 ) years were included. Anxiety and depression by BAl and $\mathrm{BDI}$ were identified in $36.9 \%$ and $58.5 \%$ of MHD patients respectively. Using the HADS, it was elicited that $35.4 \%$ of our MHD patients had anxiety and $80 \%$ had depression. The global mean score of SF-36 was $45 \pm 11.69$ with Physical Component Score (PCS) of 39.60 \pm 1.22 and a Mental Component Score (MCS) of 51.79 \pm 1.41 , indicating the poor quality of life our patient. The mean Kidney Disease Component Score (KDCS) was 65.47 \pm 11.7 . The HADS-anxiety, HADS-depression, BAI and BDI scores were significantly and inversely correlated with almost every KDQOL- ESRD and SF-36 component score $(p<0.05)$.

Conclusion: Anxiety and depression are two most prevalent entities among haemodialysis population. Anxiety and depression are associated with a lower quality of life. 


\title{
Abstract 56:
}

\section{SHORT TERM EVOLUTION OF ECHOCARDIOGRAPHIC PARAMETERS IN PATIENTS NEWLY ENROLLED FOR CHRONIC HAEMODIALYSIS}

\author{
Ingrid S. BALEMAKEN ${ }^{1}$, Jérôme BOOMBHI ${ }^{1,2}$, Hamadou BA $\hat{A}^{1,3}$, Gloria ASHUNTANTANG ${ }^{1,2}$ \\ ${ }^{1}$ Faculty of Medicine and Biomedical Sciences, University of Yaounde I, Cameroon; ${ }^{2}$ Yaounde General Hospital, \\ Yaounde, Cameroon; ${ }^{3}$ Yaounde Central Hospital, Yaounde, Cameroon.
}

Objective: Our aim was to determine the short-term effect of haemodialysis on echocardiographic parameters in patients newly enrolled for chronic haemodialysis.

Methods: We conducted a prospective cohort study which lasted 6 months (December 2016 to May 2017). Included were all consenting patients, newly diagnosed stage 5 chronic kidney disease (CKD) and admitted to chronic haemodialysis in one of the 2 haemodialysis centres in the city of Yaounde. For each patient, a full blood count, serum calcium, phosphoremia and LDL cholesterol levels were measured immediately after the indication for dialysis was posed; then transthoracic echocardiography (TTE) was performed by a cardiologist prior to the inaugural session. The same procedure was repeated 2 months later. Data analysis was done using CS pro 6.3 and SPSS 20.0 software. The threshold of significance retained was $p<0.05$.

Results: 20 patients were included, $80 \%$ of whom were male. The average age was 44 years old. The main comorbidities were high blood pressure (75\%) and diabetes (30\%). The average hemoglobin level was $7.6 \mathrm{~g} / \mathrm{dl}$ for a $100 \%$ prevalence of anaemia. Phosphocalcic disorders were hyperphosphoremia $(85 \%)$ and hypocalcemia $(55 \%)$. The prevalence of abnormalities during TTE at baseline was $100 \%$ with predominantly diastolic dysfunction (95\%), left ventricular (LV) dilation (80\%), left atrial dilation (LA) (70\%) and LV hypertrophy (60\%). The change in TTE parameters at 2 months was marked by a regression of LV mass $(p=0.021)$ and LA diameter $(p=0.031)$. In addition, an increase in diameter of the inferior vena cava at expiration $(p=0.02)$ was noted. Conclusion: Haemodialysis improves diastolic function of the LV at short-term and decreases LA size and LV mass.

\section{Abstract 57: \\ PREVALENCE AND DETERMINANTS OF SUB-CLINICAL ISCHEMIC HEART DISEASE IN PATIENTS ON MAINTENANCE HEMODIALYSIS IN YAOUNDÉ}

Cedric Xavier Mbobnda, Christian Ngongang Ouankou, Dorothee Soh, François Kaze

Department of internal Medicine and specialties of Faculty of Medicine and Biomedical Sciences, University of Yaoundé 1 , Cameroon.

Objective: Identify the prevalence and determinants of sub-clinical Ischemic heart disease in-patients on maintenance hemodialysis (MHD) in Yaoundé.

Methods: We carried out a cross sectional study on 15 patients on MHD for at least three months and above 18 years, at the Yaoundé University Teaching Hospital (YUTH) HD unit, without contraindication to exercise. Sociodemographic data, dialysis, clinical and paraclinical parameters were recorded, resting electrocardiogram (ECG) and echocardiography coupled with exercise stress testing used to identify patients with sub-clinical IHD and stratify risk of coronary artery disease (CAD).

Results: The median age was 46.77 years [ $25^{\text {th }}$ percentile $2675^{\text {th }}$ percentile 63], 1.2:1 sex ratio. Chronic glomerulonephritis was the most prevalent baseline nephropathy in. Hypertension (92.3\%) and diabetes (15.4\%) were the main comorbidities. The median duration on dialysis was 45.3 months $\left[25^{\text {th }}\right.$ percentile $975^{\text {th }}$ percentile 140 ], and $84.6 \%$ dialyzed with arteriovenous fistula. Left ventricular hypertrophy (69.2\%) was the most common resting ECG and echocardiographic abnormality. Only 13 patients completed exercise stress testing among which 10/13 classified as high risk for CAD. The sub-clinical IHD was found in 2/13, characterized by reduction of LVEF during exercise, and one of them had a regional wall motion abnormality. High serum calcium was associated with high risk CAD $(P=0.009)$. None of the factors tested were associated with IHD ( $p>0.05)$.

Conclusion: Maintenance hemodialysis patients are at higher risk of CAD with nearly one out of five patients with IHD suggesting the relevance of investigating this disease in such patients. 


\title{
Abstract 58: \\ BURNOUT SYNDROME IN HEMODIALYSIS HEALTH WORKERS IN CAMEROON: PREVALENCE AND ASSOCIATED FACTORS
}

\author{
HERMINE D FOUDA ${ }^{1,2}$, ADRIEN NGASSA ${ }^{1}$, MARIE PATRICE HALLE ${ }^{1,3}$, DENIS G TEUWAFEU ${ }^{4}$, ALEX MAMBAP T $^{5}$, HILAIRE $^{2}$ \\ DJANTIO ${ }^{3}$, FRANCOIS F KAZE ${ }^{2,6}$, GLORIA ASHUNTANTANG ${ }^{2,7}$ \\ 1 General Hospital of Douala; 2 Faculty of Medicine and Biomedical Sciences of Yaoundé- University of Yaounde l; 3 \\ Faculty of Medicine and Pharmaceutics Science- Douala; 4 Faculty of Health Sciences - Buéa; 5 Regional Hospital of \\ Bamenda; 6 Teaching Hospital of Yaoundé; 7 General hospital of Yaoundé.
}

Background: Hemodialysis medical staffs usually work in a stressful milieu. In developing countries, professional conditions are worse and can lead to burnout syndrome. However, few data are available regarding the prevalence of burnout syndrome among hemodialysis health care workers in low income countries such as Cameroon.

Objective: Determine the prevalence of burnout syndrome and its associated factors in hemodialysis health care workers in Cameroon.

Methods: We conducted a cross sectional study in all hemodialysis centers from Cameroon between January to August 2017. Data were collected from nurses and physicians using a questionnaire defining the socio- demographic characteristics and working conditions of the workers. The Maslach Burnout Inventory was used for assessment of burnout level. Burnout syndrome was defined as the presence of emotional exhaustion, depersonalization or decreased professional achievement according to the MBI score.

Results: A total of 92 health workers (women 60\%, $n=55$ ) among 106 identified were included. The median age was 42 years. Most of the workers were nurses (78.5\%) and $8.5 \%$ were nephrologists. The median time working in hemodialysis was 5 years and the median weekly working hours was 40 hours. Burnout syndrome was found in 76 (82.5\%) workers: 35 (46\%) had emotional exhaustion, 44 (58\%) depersonalization and 57 (75\%) decreased professional achievements. Burnout was significantly more prevalent in overcrowded centers (100\% Vs $47 \% \mathrm{p}<0.001)$. Burnout workers more frequently complained of headache, back pain and sleep disorders. Seniority in dialysis $>5$ years (OR 1.43 [1.01-2.03] $\mathrm{p}=0.042$ ) and desire to change position (OR 17.28 [1.25-23.96] $\mathrm{p}=0.034)$ were associated with burnout syndrome.

Conclusion: Burnout syndrome is very common among Cameroonian hemodialysis medical staff. Limiting positions in hemodialysis centers $<5$ years and change of position when requested are potential preventive measures.

\section{Abstract 59:}

\section{CHRONIC KIDNEY DISEASE IN SICKLE CELL DISEASE: PREVALENCE AND ASSOCIATED FACTORS IN THE ECONOMIC CAPITAL OF CAMEROON}

HERMINE D FOUDA ${ }^{1,2}$, GILLES WANDJI ${ }^{1}$, DENIS G TEUWAFEU ${ }^{3}$, MARIE PATRICE HALLE ${ }^{1,4}$ ALEX MAMBAP T $^{5}$, HILAIRE DJANTIO ${ }^{4}$, FRANCOIS F KAZE ${ }^{2,6}$, GLORIA ASHUNTANTANG ${ }^{2,7}$

1 General Hospital of Douala; 2 Faculty of Medicine and Biomedical Sciences of Yaoundé- University of Yaoundé l; 3 Faculty of Health Sciences - Buéa; 4 Faculty of Medicine and Pharmaceutics Science-Douala; 5 Regional Hospital of Bamenda; 6 Teaching Hospital of Yaoundé; 7 General hospital of Yaoundé.

Background: Previous studies in Cameroon have reported a high prevalence of proteinuria (93\%) and urinary abnormalities (78\%) in sickle cell anemia (SCA). However, the exact prevalence of chronic kidney disease (CKD) in SCA is not known since the persistence of these anomalies at 3 months was not evaluated. Moreover, the prevalence of CKD in people with Sickle cell trait (SCT) in Cameroon has not been studied.

Objective: Evaluate the prevalence of CKD in people with sickle cell disease in the main SCA health facility of the economic capital of Cameroon.

Methods: SCA patients were recruited during routine visits between April $1^{\text {st }}$ and September $30^{\text {th }} 2017$ at Douala Laquintinie hospital. SCT patients in the family (parents or siblings) of homozygote patients were also recruited. CKD was diagnosed and classified according to 2012 KDIGO criteria. Only patients with persistent urinary anomalies or decreased eGFR at 3 months were considered to have CKD.

Results: A total of 107 patients including 81 SCA (men 62\%) and 26 SCT (men 46\%) participated in this study. SCA patients were younger (17 years Vs 35 years, $p<0.001$ ) with lower BMI, systolic and diastolic BP. Overall, CKD was found in 16 patients (15\%): 13 patients (16\%) in the SCA group and $3(11 \%)$ in the SCT group. CKD frequency was comparable in the both groups $(p=0.76)$. Albuminuria was more common in the SCA group $(12 \neq 1$ patient, $p=0.018)$. 
Age $\leq 25$ years was protective factor for both CKD (OR $=0.20$ [0.003-0.135], $p<0.001)$ and albuminuria $(O R=0.23$ [0.004-0.124], $p<0.001$ ) in SCD group.

Conclusion: CKD is less prevalent in our setting compared to previous studies in Cameroon and sub-Saharan Africa.

\section{Abstract 60: \\ FACTORS ASSOCIATED WITH PULMONARY HYPERTENSION IN PATIENTS ON MAINTENANCE HEMODIALYSIS AT THE YAOUNDE GENERAL HOSPITAL}

Mahamat M*, Mahama A¹, V. Nzana', K. Mukwelle², A. Nono², J. Boohmbi', G. Ashuntantang ${ }^{1}$

1: Department of Internal Medicine and Specialties, Faculty of Medicine and Biomedical Sciences, University of Yaoundé 1, Yaoundé, Cameroon; ${ }^{2}$ : Hemodialysis unit, Yaoundé General Hospital, Yaoundé, Cameroon.

Objectives: To determine factors associated with pulmonary hypertension in patients on maintenance hemodialysis in an urban sub-Saharan hospital.

Methods: We conducted a cross sectional study from November 2017 to May 2018. We included all consenting adult patients on hemodialysis for more than 3 months. Patients with a history of congenital cardiopathies, pulmonary or valvular diseases, were excluded. Transthoracic echocardiography was performed 24 hours after the dialysis session. The maximum velocity of tricuspid regurgitation flux on the Doppler was used to obtain the pulmonary arterial pressure using the modified Bernoulli equation. Pulmonary hypertension $(\mathrm{PH})$ was defined as a pulmonary arterial pressure (PAP) $>35 \mathrm{mmHg}$ and considered severe when $>60 \mathrm{mmHg}$. Calcium and phosphorous were measured in arterial blood samples prior to mid-week dialysis session. A value of $p<0.005$ was considered significant. Ethical clearance was obtained from the hospital ethics board.

Results: From the 108 included patients, $57.4 \%(n=62)$ were males and the mean age was $48.2 \pm 14.5 y e a r s$. The major cardiac abnormality was left ventricular hypertrophy (LVH). The prevalence of PH was $43 \%$ ( $n=46)$. The mean PAP was $46.3 \pm 10.1 \mathrm{mmHg}$ with severe $\mathrm{PH}$ observed in 6 patients. In the PH group, mean age was $46.2 \pm 15.2$ years and $53.4 \%$ were males $(n=25)$. LVH, left and right atrial dilatation were observed in $82.6 \%(n=38), 37 \%(n=17)$ and $23.9 \%(n=11)$ patients respectively. These 3 were all independent risks factors associated with a PH $(0 \mathrm{R} 3.2 ; 6.2$ and 7.9 respectively). Eighty-eight percent $(n=93)$ had a hemoglobin $(H b)$ level $<8 g / d l$ with levels less than $7 g / d l ~(32.6 \% ; n=15)$ being significantly associated ( $p=0.035$; OR 1.74) with $\mathrm{PH}$. Conclusion: $\mathrm{PH}$ is prevalent in hemodialysis patients and it is associated with anemia and cardiac abnormalities.

\section{Abstract 61:}

\section{THE HEART OF UREMIC AT THE INITIATION OF DIALYSIS}

Yao K. Hubert; Diopoh S. Patrick; Dje Bi Landry; Konan S. Didier; Aka A. Jean; Niava Randolphe; Sanogo Sindou Department of Nephrology-Internal Medicine D CHU Treichville.

Introduction: In the dialysis, the main cause of morbidity and mortality is cardiovascular.

Objective: To describe the cardiac status of patients at initiation of dialysis

Methods: This was a descriptive prospective cohort study conducted over a period from January 2016 to December 2018

Results: The prevalence of cardiac abnormalities was $89.6 \%$; sex ratio: 1.53; Mean age: $39.31 \pm 14.89$; comorbidities were: hypertension (73.5\%), kidney desease (23.8\%), heart disease (15\%), diabetes (10.9\%); the main signs were: renal failure (61.2\%), dyspnea (14.3\%); the main clinical syndroms: hypertension (89.8\%), edema (73.5\%), anemia (47.6\%); HTA was grade III in 64\% of patients; anemia was severe in 55.8\%; echocardiographic abnormalities observed were: concentric hypertrophy (57.1\%), eccentric hypertrophy (34\%), pericarditis (32\%), LVEF (55.1\%); kidney failure was: chronic (93.9\%), acute (5.4\%); renal involvement was glomerular (51\%), vascular (34\%); the causes of end-stage renal failure were: chronic glomerulonephritis (34.7\%), hypertension (34\%); HTA causes: essential (50.3\%), glomerular disease $(46.9 \%)$, renal function: recovery $(10.9 \%)$, stationary $(48.3 \%)$; The mortality was $40.8 \%$; In univariate analysis, there appeared to be a statistical relationship between hypertension $(O R=0.386,95 \% \mathrm{Cl}=0.173-0.86, p=0.017)$ and the risk of developing concentric hypertrophy, whereas edematous syndrom $(O R=2.625, \operatorname{IC95} \%=1.087-6.340, p=$ $0.022)$ and pulmonary edema $(\mathrm{OR}=3.094,95 \% \mathrm{Cl}=1.496-6.4, \mathrm{p}=0.002)$ appeared to be statistically associated with eccentric hypertrophy; HTA grade $3(\mathrm{OR}=2.357,95 \% \mathrm{Cl}=1.119-4.96, \mathrm{p}=0.019)$ and impaired $\operatorname{LVEF}(\mathrm{OR}=0.59,95 \% \mathrm{Cl}$ $=0.47-0.75, p=0.001$ ) appeared statistically associated with concentric hypertrophy; in altered LVEF multivariate 
analysis $(\mathrm{OR}=2.17,95 \% \mathrm{Cl}=1.14-4.11, \mathrm{p}=0.017)$ and age $<35$ years $(\mathrm{OR}=2.56,95 \% \mathrm{Cl}=1.32-4,95, \mathrm{p}=0.005)$ were associated with the occurrence of deaths.

Conclusion: Heart disease is common at the initiation of dialysis. Early detection improves the prognosis.

\section{Abstract 62: \\ DIALYSIS OUTCOMES AND PRACTICE PATTERNS AMONG CHRONIC KIDNEY DISEASE PATIENTS RECEIVING HEMODIALYSIS AT A PRIVATE DIALYSIS CENTRE IN NAIROBI, KENYA BETWEEN MAY 2016 AND JULY 2016}

Odhiambo $\mathrm{JO}^{1}$, Twahir $\mathrm{A}^{2}$

1 Maseno University; 2 Parklands Kidney Centre.

Background: Chronic Kidney Disease is a long term condition caused by damage to both kidneys. It has become a major public health concern worldwide in the past decade and its prevalence is projected to rise in the coming years. Owing to its irreversible nature, renal transplantation has been proven to be the most effective renal replacement therapy. However, it is limited by its high cost and low availability of donor kidneys. Hemodialysis has therefore been adopted widely in Kenya as an alternative therapy for those with End Stage Renal Disease. It partly compensates for the loss of renal function and its associated sequelae.

Objective: To determine dialysis outcomes and practice patterns for Chronic Kidney Disease patients receiving hemodialysis at a private centre in Nairobi, Kenya.

Methods: This was a retrospective study. Records of 43 patients who received dialysis at the centre for more than six months were reviewed and the mid-year results for different parameters obtained.

Results: $62.8 \%$ were male, $51.2 \%$ were Africans with the rest being Asian. The mean age of the patients was 63years $( \pm 16) .79 .1 \%$ were married. $60.5 \%$ had both hypertension and diabetes. Arteriovenous Fistula (81.4\%) and Tunneled catheter (18.6\%) were the only routes used for vascular access. Majority of the patients $(72.1 \%)$ received dialysis twice weekly. $93.0 \%$ and $97.7 \%$ were on iron and erythropoietin supplementation respectively. Most patients (39.53\%) had hemoglobin of $10-10.99 \mathrm{~g} / \mathrm{dl}$. Only $9.3 \%$ had completed the whole course of hepatitis B vaccination. $72.1 \%$ had $\mathrm{HBsAb}$ titres $>10 \mathrm{U} / \mathrm{ml}$. 26.6\%, 50\% and $29.3 \%$ had normal calcium, phosphate and parathyroid hormone levels respectively.

Conclusion: Majority of the hemodialysis patients were males and aged above 60 years; Diabetes and Hypertension were the most common co-morbidities among hemodialysis patients at the centre; Majority of the patients had Hemoglobin levels below $12 \mathrm{~g} / \mathrm{dl}$ despite erythropoietin and Intravenous Iron supplementation.

\section{Abstract 63:}

\section{AN ECHOCARDIOGRAPHIC EVALUATION OF PULMONARY PRESSURES IN HEMODIALYSIS PATIENTS AT KENYATTA NATIONAL HOSPITAL}

Soki KB, Were AJO, Ogola ES, Nyale GM, Murage MM

Background: A high prevalence of pulmonary hypertension (PH) in patients with end-stage renal disease (ESRD) has been documented in previous studies. In these patients, $\mathrm{PH}$ increases morbidity and mortality and worsens prognosis post-renal transplant but its aetiopathogenesis is poorly understood. This study aimed to determine the prevalence of pulmonary hypertension $(\mathrm{PH})$ in ESRD patients undergoing chronic hemodialysis at Kenyatta National Hospital and to explore possible associations between $\mathrm{PH}$ and a number of clinical and echocardiographic variables.

Methods: A cross-sectional descriptive study between February and April 2015 to determine the prevalence of PH in ESRD patients at renal unit KNH using Doppler echocardiography. Data was collected on preformed sheets and analyzed.

Results: A total of 117 patients were recruited over seven weeks. 63.2\% were male and the mean age of patients was 44 years. Prevalence of $\mathrm{PH}$ among ESRD patients at the renal unit $\mathrm{KNH}$ was $32.5 \%$, with a median PASP of $47.3 \mathrm{mmHg}$ and a range of 36.1-79 $\mathrm{mmHg}$. A strong association between $\mathrm{PH}$ and $\mathrm{EF}$ of less than $50 \%$, as a marker of LV dysfunction, was demonstrated.

Conclusion: The prevalence of PH among ESRD patients at renal unit KNH is high. A significant association was found between $\mathrm{PH}$ and left ventricular dysfunction. This may be a significant point of intervention in the reduction of morbidity and mortality in this population 


\section{Abstract 64: \\ DIETARY PRESCRIPTION ADHERENCE AMONG ADULTS WITH CHRONIC KIDNEY DISEASE ON HEMODIALYSIS}

Rose Opiyo ${ }^{1,2}$, Anthony Were ${ }^{2,3}$, Zippora Bukania ${ }^{4}$, Esther Nabakwe ${ }^{5}$, Alexander Mbogo ${ }^{6}$, Joyce Olenja $^{1}$ ${ }^{1}$ School of Public Health, College of Health Sciences, University of Nairobi, Nairobi, Kenya; ${ }^{2}$ East African Kidney Institute, College of Health Sciences, University of Nairobi, Nairobi, Kenya; ${ }^{3}$ Department of Internal Medicine, College of Health Sciences, University of Nairobi, Nairobi, Kenya; ${ }^{4}$ Centre for Public Health Research - KEMRI, Nairobi, Kenya; ${ }^{5}$ Department of Child Health and Paediatrics Moi University, Eldoret, Kenya; ${ }^{6}$ Kenyatta National Hospital, Nairobi, Kenya.

Objective: The study documented dietary prescription adherence levels and associated factors among adults with Chronic Kidney Disease on hemodialysis at national referral and teaching hospitals in Kenya.

Methods: A mixed-methods research design with quantitative survey as the primary method was conducted at the renal clinics and dialysis units within Kenyatta National Hospital and Moi Teaching and Referral Hospital. Adult patients with chronic kidney disease on hemodialysis without kidney transplant were purposively sampled for the quantitative survey. A sub-sample of adult patients and their caregivers were also purposively sampled for qualitative survey. The outcome variable, 'adherence to diet prescription', was collected on a 5-point Likert Scale, but analyzed as a binary variable. Logistic regression analysis was conducted for quantitative data while qualitative data were thematically analyzed.

Results: Of the 333 participants in the quantitative survey, $92.7 \%$ were aware of renal diets. However, only $36.3 \%$ of those aware of the renal diets adhered to their dietary prescriptions. Factors that were independently associated with adherence to the diet prescriptions were 'flexibility in the diets to combine with other ways of eating' (OR:2.65,95\% $\mathrm{Cl}: 1.11$ - 6.30, P:0.028); 'difficulties in following diet recommendations' (OR:0.24, 95\% $\mathrm{Cl}: 0.13-0.46, \mathrm{P}<001)$ and 'adherence to limiting fluid intake' (OR:9.74, 95\% Cl:4.90 - 19.38, P<0.001).

Conclusion: Adherence to dietary prescription among hemodialysis patients is low despite awareness of dietary restrictions. Diet prescriptions with less restrictive foods that require minimal extra efforts and resources are more likely to be adhered to than the restrictive ones.

\section{Abstract 65:}

\section{DETERMINATION OF CONCORDANCE BETWEEN BIOIMPEDANCE ANALYSIS AND A CLINICAL SCORE IN FLUID STATUS ASSESSMENT OF PATIENTS ON MAINTENANCE HEMODIALYSIS}

\section{K. Muchiri, J.K. Kayima, E.N. Ogola, S.O. McLigeyo}

Background: The burden of chronic kidney disease (CKD) is rising rapidly globally. Fluid overload (FO), an independent predictor of mortality in CKD, must be accurately quantified to enable maintenance of normohydration. Clinical assessment is widely used to determine FO could result in underestimation in fluid overload. Conversely, bioimpedance analysis (BIA) has been shown to be accurate and reproducible in determining fluid status of CKD patients on haemodialysis (HD). However, it is unclear which of the two methods is more sensitive in assessing volume status in our population.

Objective: To assess the hydration status of maintenance HD (CS) patients using BIA and assess the level of agreement between BIA and a clinical score in fluid status assessment.

Methodology: This was a single centre hospital based cross-sectional analytic study which recruited a sample of 80 CKD patients at the renal unit of Kenyatta National Hospital. Included patients were at least 18 years old, on maintenance HD, without a pacemaker, metallic implant or bilateral limb amputation. Data on the patients' clinical history, physical examination and chest radiography findings were filled into a questionnaire and BIA done on each participant.

Results: A high proportion of participants had FO (88.75\%) with mean excess extracellular volume being 3.02L $\pm 1.79 \mathrm{~L}$. There is no difference in the proportion of patients diagnosed to have fluid overload using BIA and the CS. The best cut-off point for the CS was a normal range of -2 to 2 with values below -2 indicating dehydration above 2 indicating fluid overload. At this cut-off point the CS had a sensitivity of $81.05 \%$ and a specificity of $19.9 \%$ while BIA had a sensitivity of $87.3 \%$ and specificity of $13.46 \%$. None of the factors assessed had a statistically significant association with fluid overload on multivariable logistic regression analysis.

Conclusion: In this population the CS had a good level of agreement with BIA with both having relatively good sensitivity but poor specificity. Further studies need to be done to check for consistency of this findings. 


\title{
Abstract 66: \\ OCULAR COMPLICATIONS IN PATIENTS UNDERGOING LONG-TERM HEMODIALYSIS AT ARISTIDE LE DANTEC UNIVERSITY HOSPITAL (SENEGAL)
}

Faye $M^{1}$, Lemrabott $A T^{1}$, Sakho $B^{1}$, Sow $A S^{2}$, Faye $\mathrm{Ms}^{1}$, Cissé $\mathrm{MM}^{3}$, Seck $\mathrm{SM}^{4}$, Kane $\mathrm{Y}^{5}$, Fall $\mathrm{K}^{1}$, Mbengue $\mathrm{M}^{1}, \mathrm{Keita}^{1}, \mathrm{Ba}$ $B^{1}$, Diagne $S^{1}$, Dieng $A^{1}$, Ba $A A^{1}$, Niang $A^{1}, K a E H F^{1}$, Diouf $B^{1}$.

1 Nephrology Department, Aristide Le Dantec University Hospital, Cheikh Anta Diop University, Dakar (Senegal); 2 Department of Ophthalmology, Cheikh Anta Diop University, Dakar (Senegal); 3 Faculty of Medicine. Thies University, Thiès (Senegal); 4 Faculty of Medicine. Gaston Berger University, Saint-Louis (Senegal); 5 Faculty of Medicine. Assane Seck University, Ziguinchor (Senegal).

Introduction: The negative impact of chronic kidney disease on the patient's eye is complex and diverse, but the effects of hemodialysis (HD) on ophthalmological parameters are unclear. The aims of this study were to explore ocular changes in chronic hemodialyzed patients and to determine the factors associated with these ocular anomalies. Patients and methods: A cross-sectional study was conducted during 4 month. Patients undergoing hemodialysis at Aristide Le Dantec University Hospital center for more than three months were included. An ophthalmological examination, with visual acuity measurement, an examination of the anterior segment to the slit lamp, and an examination of the fundus were performed.

Results: 55 patients ( $43.6 \%$ men, $56.4 \%$ women) were included with median age of 47 years [extemes $21-71$ years]. The mean duration on hemodialysis was $67.43 \pm 39.05$ months. 110 eyes were examined: decreased visual acuity was found in $36.4 \%$ with $2.7 \%$ cases of blindness. Cataracts was present in $33.6 \%$, glaucoma in $3.6 \%$, retinopathy in $48.8 \%$, and bilateral gerontoxon in one patient. Arteriosclerosis and macular atrophy were found in $29.3 \%$. Dry eye was found in $11 \%$ and pterygium in $3.6 \%$. Refractive anomalies were found with isolated myopia in $17.4 \%$, myopia in combination with astigmatism in $47.8 \%$, isolated hyperopia and presbyopia in $8.7 \%$, and hyperopia in combination with astigmatism in $8.7 \%$. Age and seniority in dialysis were correlated with cataract occurrence.

Conclusion: Ocular complications in hemodialysis patients are variable. The ocular lesions found were frequent and cannot be attributed to hemodialysis. Patients should have routine eye examinations to detect and treat these lesions before the complications stage.

\section{Abstract 67:}

\section{THE ELDERLY SUBJECT IN CHRONIC HEMODIALYSIS: EXPERIENCE OF 2 SEMI-URBAN UNITS IN SENEGAL}

\author{
KANE Yaya1 *, LEMRABOTT A T2, FAYE M2, FAYE Mo1, CAMARA MS4, CISSE M M4, SECK S M3, KA EI F2, NIANG A2, \\ DIOUF B2.
}

1 Nephrology service hemodialysis-gastroenterology-internal medicine peace hospital Assane University Seck ZIGUINCHOR; 2 Hemodialysis Nephrology Department HALD UCAD Dakar; 3 Hemodialysis Nephrology Service CHR of St Louis Gaston Berger University; 4 Nephrology service hemodialysis CHR Thiès / Thiès University.

Introduction: More and more elderly patients are being treated for chronic hemodialysis. The objective of our study is to describe the epidemiological and clinical profile of elderly patients treated with chronic hemodialysis.

Patients and methods: This is an observational, transversal, multicenter study conducted for 1 month, from April 1st to April 30th, 2019, including all chronic hemodialysis patients from the Ziguinchor and Thiès units. We analyzed the demographic, clinico-biological and dialytic parameters in 65-year old patients or more.

Results: Among 77 chronic hemodialysis patients currently treated in our 2 units, 17 patients are 65 years old or more, a prevalence of $0.22 \%$. The average age of patients was 67.4 years old and extremes of 65 and 73 years old. There were 8 women (47.1\%) and 9 men (52.9\%). Causal nephropathy was vascular in 12 patients (70.6\%), indeterminate in 3 patients (17.6\%), glomerular and chronic tubulointerstitial respectively in 1 patient (5.9\%) for everyone. The average duration of hemodialysis was 53.4 months [10-132 months]. Six patients (35.3\%) had professional activities. Twelve patients $(70.7 \%)$ had vascular access to a native arteriovenous fistula. Comorbidities were noted in 13 patients (76.74\%) dominated by high blood pressure in 16 patients followed by cardiovascular disease in 3 patients. Five patients (29.41\%) had cognitive impairment. Twelve patients $(70.59 \%)$ needed assistance in their daily activities by a third party. The average hemoglobin rate was $8.17 \mathrm{~g} / \mathrm{dl}$. Dialysis was well tolerated in 12 patients (70.59\%). The majority of patients (88.24\%) were on antihypertensive therapy.

Conclusion: The elderly in chronic hemodialysis represent a population of fragile patients. Special and regular monitoring is necessary to prevent complications and improve their quality of life. 


\title{
Abstract 68:
}

\section{USEFULNESS OF BIOELECTRICAL IMPEDANCE ANALYSIS FOR ASSESSING VOLUME STATUS IN CHRONIC HEMODIALYSIS PATIENTS AT THE ARISTIDE LE DANTEC UNIVERSITY HOSPITAL (SENEGAL)}

\author{
Keita $\mathrm{N}^{1}$, Lemrabott $\mathrm{AT}^{1}$, Seck $\mathrm{SM}^{2}$, Faye $\mathrm{M}^{1}$, Faye $\mathrm{Ms}^{1}$, Cissé $\mathrm{MM}^{3}$, Fall $\mathrm{K}^{1}, \mathrm{Ba} \mathrm{B}^{1}$, Diagne $\mathrm{S}^{1}$, Mbengue $\mathrm{M}^{1}$, Dieng $\mathrm{A}^{1}$, Ba \\ $A A^{1}$, Niang $A^{1}, K^{2} E_{H F}{ }^{1}$, Diouf $B^{1}$. \\ 1 Nephrology Department, Aristide Le Dantec University Hospital, Cheikh Anta diop University, Dakar (Senegal); 2 \\ Faculty of Medicine. Gaston Berger University, Saint-Louis (Senegal); 3 Faculty of Medicine. Thies University, Thiès \\ (Senegal).
}

Introduction: Determination of dry weight is one of the daily goals to achieve in hemodialysis. The objective of this work was to validate the use of bioelectrical impedance analysis (BIA) in estimation of dry weight.

Methods: This is a 9-week cross-sectional and analytical study, from August 01 to September 31, 2017, at the hemodialysis unit of Aristide Le Dantec University Hospital. Included were consenting patients over the age of 18 who had been on chronic hemodialysis for more than 3 months and no history of hospitalization in the last month. Without modifying the clinically established dry weight, we measured weight and total body water by using Tanita's bathroom scale (BIA technology) before and after hemodialysis session for 6 successive sessions. These measures were compared with results from clinical measures. Comparison of the repeated measurements was performed using a Student's t-test on paired samples and the agreement was evaluated by linear regression and Brand-Altman analysis. Results: 264 measurements were performed in 22 patients. The average age was $46.6 \pm 13.1$ years, with $54.5 \%$ of men. During the hemodialysis session, there was a significant reduction in weight $(65 \pm 17.1 \mathrm{~kg}$ pre-dialysis compared with $62.9 \pm 17.0 \mathrm{~kg}$ post-dialysis, $p<0.0001$ ) and total body water (TBW) measured by BIA (TBWBIA $=36.3 \pm 7.1 \mathrm{~L}$ pre-dialysis versus $33.0 \pm 6.8 \mathrm{~L}$ post-dialysis, $\mathrm{p}<0.0001$ ) or calculated by the Watson equation (TBWWatson $=35.8 \pm 6.9 \mathrm{~L}$ pre-dialysis against $35.2 \pm 6.8 \mathrm{~L}$ post-dialysis, $p<0.0001$ ). We found a strong linear correlation and a concordance between the 2 TBW measurements in predialysis. This correlation remained high in non-concordance postdialysis with a mean of -2.2 differences, a very wide agreement limit $(-5.9$ and +1.5$)$, and a significant difference in measurements.

Conclusion: These results demonstrate the reproducibility, reliability and repeatability of impedance metric measurements in our chronic hemodialysis patients.

\section{Abstract 69:}

\section{SURVIVAL OF SOUTH AFRICAN PATIENTS ON RENAL REPLACEMENT THERAPY}

T Jardine ${ }^{1}$, Esther Wong ${ }^{2}$, Retha Steenkamp ${ }^{2}$, FJ Caskey ${ }^{2}$, MR Davids ${ }^{1}$

${ }^{1}$ Division of Nephrology, Stellenbosch University and Tygerberg Hospital, and South African Renal Registry, Cape Town, South Africa; ${ }^{2}$ United Kingdom Renal Registry and University of Bristol, Bristol, UK.

Background: The aim of this study was to evaluate, for the first time, the survival of South African patients on RRT based on data from the South African Renal Registry (SARR).

Methods: SARR data were used to estimate 1-year survival in patients on RRT. The incident cohort included patients who initiated treatment between January 2013 and December 2016. The prevalent cohort included those on RRT on 31 December 2015. Data were collected on potential risk factors for mortality. Failure events included stopping treatment without recovery of renal function, and death. Patients were censored if they were alive on RRT at 1 year, or recovered renal function or were lost to follow-up. The Kaplan-Meier method was used to estimate one-year survival. Multivariable Cox regression was used to examine the association of potential risk factors with survival in incident patients, while logistic regression was used in prevalent patients.

Results: In the incident cohort ( $\mathrm{n}=6187$ ), the mean age was 51.8 years, $54 \%$ were black, $44 \%$ were diabetic, $10 \%$ were HIV positive, and $82 \%$ had haemodialysis (HD) as their first RRT modality. Overall 1-year survival was $90.4 \%(95 \% \mathrm{Cl}$ 89.6-91.2). The 1-year survival was independently associated with age, renal diagnosis and province of residence. There were no differences based on ethnicity, diabetic status or healthcare sector. One-year survival was $95.7 \%$ and 93.8\% in HIV-positive and HIV-negative patients, respectively. One-fifth had no data on HIV-status and the survival in this group was considerably lower at $74.8 \%(p<0.001)$.

In the prevalent cohort $(n=10155)$, baseline characteristics and one-year survival were similar to that of the incident group. Mortality was independently associated with age, ethnicity, diabetes, province of residence and RRT vintage. Conclusions: The survival rates of South African patients who access chronic dialysis and transplantation services are comparable to those in better-resourced countries. It is still unclear what effect, if any, HIV status has on patient survival. 


\title{
Abstract 70:
}

\section{INDICATIONS AND LATE RESULTS OF PARATHYROIDECTOMY FOR HYPERPARATHYROIDISM IN PATIENTS UNDER HEMODIALYSIS: A SERIES OF 18 CASES}

\author{
R.BENHAMOU, M. CHETTATI, H. FENNANE, Y.MSOUGAR, W. FADILI, I. LAOUAD \\ Mohammed VI University center of Marrakesh, Cadi Ayyad University, Marrakesh, Morocco.
}

Objective: We sought to describe different characteristics of patients with secondary or tertiary hyperparathyroidism receiving surgical treatment under hemodialysis, and to compare outcomes between different treatment strategies. Methods: We conducted a prospective single institution cohort study of patients aged $>18$ year and on dialysis who received a parathyroidectomy between May 2017 and 2019.

Procedures were classified as subtotal parathyrdoidectomy (sPTX), and total \pm autotransplant(tPTX). The primary outcome variable of interest was 30-day outcome of the surgical procedure (PTH and calcemia normalization at one week and one month postoperatively). Secondary outcome variables studied were operative time, postoperative length of stay (LOS), 30-day mortality, and 30-day readmission. Multivariate analyses were performed.

Results: A total of 18 patients studied. The mean age was $42,89 \pm 17,95 \mathrm{y}$. Before receiving PTX mean parathyroid hormone value was $2496,88 \pm 834,9$ IU (Cobas 6000, Roche Diagnostics). Ten patients (55,6\%) received a subtotal parathyroidectomy. Mean operative time in both surgical techniques was $135 \pm 45$ min and median LOS 6 days for subtotal parathyroidectomy versus 11 days for total parathyroidectomy $(p<0,05)$. The overall complication rate was $22,2 \%$, and $33,3 \%$ of patients $(p>0,05)$. There was no difference in the 30 -day morbidity, mortality, or readmission rates between the two treatments.

Conclusion: Successful surgical intervention for SPTX and tPTX significantly reduces preoperative symptoms and leads to restoration of bone disease. In our experience total PTX with autograft has proven to be a satisfactory procedure. Subtotal PTX is also an effective procedure and the choice of operative technique should be left to the surgeon.

\section{Abstract 71:}

\section{CHALLENGES OF HAEMODIALYSIS: A SINGLE CENTRE EXPERIENCE IN SOUTH WEST NIGERIA}

Dada, Samuel Ayokunle

Nephrology Unit Department of Medicine, Ekiti State University Teaching Hospital, Nigeria.

Background: Haemodialysis is the commonest method of Renal Replacement Therapy in Nigeria. Despite an advancement in the technicality and better understanding of haemodialysis, a number of challenges and complications are known to be associated with this procedure especially in resource poor nations Objective: We aimed to highlight our experiences and share some of the uncommon complications encountered during haemodialysis and present the outcome of our patients.

Subjects and methods: A retrospective review of 101 patients during the last two years was done. Data extracted include sociodemographic characteristic, aetiology of kidney disease, type of vascular access, intradialytic complication and outcome of treatment.

Results: The total number of dialysis session during the period was 823 . Males constituted a higher proportion (64.4\%) and were found to be older than female patients 49.8 vs 42.8 years ( $P=0.001)$. Majority ( $89.1 \%$ ) had chronic kidney disease while chronic glomerulonephritis was the main cause of CKD as seen in about $45 \%$ of the patient. Due to the cost implication, only 2(1.98\%) were able to undergo 3 sessions of dialysis per week for up to 1 month. Vascular access was femoral (66.3\%), internal jugular vein (25.7\%), while only $2 \%$ used Artero-venous-fistula and one patient had femoral vessel pseudoaneurysm from frequent cannulation. The commonest complication was hypotension which was present in $15.8 \%$. Twenty-eight deaths were recorded, $44(43 \%)$ were either lost to follow up or absconded while $5 \%$ were transplanted at a referral centre.

Conclusion: Challenges of renal replacement therapy is overwhelming in our country due to poor human and financial resources. Early diagnosis and adequate government support are advocated. 


\section{PERITONEAL DIALYSIS}

\section{Abstract 72: \\ POSTERIOR URETHRAL VALVES IN THE SECONDARY HOSPITAL OF DOUALA IN CAMEROON: CLINICAL, RADIOLOGICAL, THERAPEUTICAL AND EVOLUTIVE ASPECTS}

\section{Kaptue Moudze B, Sala T., Moguem L.}

Laquintinie Hospital of Douala Cameroon.

Objectives: To determine the clinical, biological, radiological and therapeutical features of PUV.

Methods: It was a retrospective study from 1st May 2014 to 31 December 2018 in the pediatric unit of Laquintinie Hospital in association with urology unit.Socio demographical, clinical, radiological and therapeutical and also evolutive data were all analysed.

Results: The records of fifteen patients were selected. Antenatal diagnoses was made in two(13\%) patients. The mean age of post natal diagnosis was 20,18 months .Oligohydramnios was found on two (13\%)patients. One of them was born at thirty four weeks gestational age.The commonest presenting clinical features were repetitive urinary tract infections $12(87 \%)$, dysuria and abnormal urinary stream $12(87 \%)$, urinary retention $8(53 \%)$, pyuria $8(57 \%)$,failure to thrive $8(53 \%)$, anemia $6(40 \%), 1(6 \%)$ has high blood pressure over than 90 th percentile,3(20\%) had proteinuria.11(73\%) had acute kydney injury (AKI) :one (6\%) stade 1,three (20\%) stade 3,four (27\%) stade 4, three $(20 \%)$ end stage renal failure (ESRF). Urinary ultra sound was made on all the patients, among them $14(93 \%)$ had bilateral hydronephrosis, and $1(7 \%)$ had unilateral hydronephrosis with one kidney atrophy. In twelve (80\%) patients, the dilatation of PUV was confirmed by retrograde urethrocystography. Ten(67\%) patients underwent vesicostomy and endoscopy resection of the valves. The mean age for surgery was 28,6 months [3-48 months].Four (27\%) died before surgery. One lost of follow up. No child benefited from dialysis.4 children progressed towards chronic kidney disease (CKD).

Conclusion: Antenatal diagnoses is important because it may improve outcome of boys with PUV.A follow -up has to be done by a multidisciplinary team during a long time to detect clinical,biological and radiological abnormalities.

\section{Abstract 73:}

\section{LAPAROTOMY ON 8 YEARS OLD BOY WITH NEPHROTIC SYNDROME}

Georgette Guemkam ${ }^{\circ}$ Vincentia Kifem, Ingara S. T. Youmbi ${ }^{\circ}$, Paul Koki ${ }^{1}$

${ }^{\circ}$ Pediatric nephrology unit, Mother and child Centre of C. Biya Foundation; ${ }^{1}$ Deparment of Pediatrics; Faculty of medicine and Biomedical Sciences.

Introduction: Nephrotic syndrome is a renal disease characterised by massive proteinuria, hypoalbuminaemia, pitting oedema to anasarca and hyperlipidemia. Spontaneous bacterial peritonitis can occur as a complication and management is medical with no need for surgery. However, peritonitis can be the first sign of presentation and if an underlying nephrotic syndrome is not suspected this patient can be operated unnecessarily.

Case presentation: An 8 years old male was referred for anasarca. He consulted 6 days ago for fever and abdominal pain. At the regional hospital (far from Yaounde, the capital), the abdominal ultrasound showed abdominal fluid collection extending to the pelvis) and malaria test positive. Emergency laparotomy was done for either appendicular peritonitis or acute cholecystitis. The child receive fluid, amoxicillin-acid clavulanic, metronidazole, ranitidine, artesunate and antipyretic. Four days post op, he develops anasarca and abdominal distension. He was referred to the paediatric nephrology unit where a diagnosis of nephrotic syndrome complicated by spontaneous bacterial peritonitis was made based on oedema and paraclinical investigations: 24 hour proteinuria $2061.5 \mathrm{mg}$; albuminemia: $15 \mathrm{~g} / \mathrm{l}$; other tests: HIV negative, Hepatitis C negative, Hep Bs Ag negative, ESR 108, CRP negative, FBC: WBC 4500, neut 2600, lymp 1400; HB 12.2g/dl, platelets 254000; pu 100, blood 5 to 10; PT /aPPT 64.5\%/42.1 s; Na/K/Cl: 128.2/3.71/98.6; Triglycerides2.71; HDL/LDL 0.46/2.16; Total cholesterol 3.62; ultrasound: moderate to marked abdominopelvic fluid collection, suspicious of peritonitis. The same treatment were continue for 10 days. Then, the child was discharge and put on prednisone $60 \mathrm{mg} / \mathrm{day} / \mathrm{m}^{2}$. One month later he presented with second episode of anasarca and signs of peritoneal irritation. He was on amoxicillin + clavulanic acid for 10 days injectable plus 10 days orally with prednisone and show relieve of peritoneal symptoms. After 6 weeks of prednisone; $24 \mathrm{hr}$ Proteinuria was $2519 \mathrm{mg} / \mathrm{day}$. He was thus switched to cyclophosphamide at $2 \mathrm{mg} / \mathrm{kg} /$ day together with prednisone $30 \mathrm{mg} / \mathrm{m} 2$ every other day for 3 months. Evolution was simple and proteinuria ranged to normal values. 
Conclusion: Laparotomy is unnecessary in case of peritonitis associated nephrotic syndrome. It should be considered in any child presenting with signs of peritoneal irritation and oedema. This child presented with peritonitis twice and treated well with amoxicillin+ clavulanic acid.

\section{Abstract 74: \\ HEMOGLOBINURIA RELATED ACUTE KIDNEY INJURY IN CHILDREN WITH G6PD DEFICIENCY: NECESSITY OF PREVENTIVE MEASURES}

Suzy Hamo, Félicitée Nguéfack, Andréas Chiabi

Gyneco obstetric and pediatric hospital of Yaounde, Cameroun.

Objectives: Describe the appropriate means to prevent factors that trigger hemolysis which leads to multiple transfusions and renal injuries

Cases reports: We described 2 cases of male patients with G6PD deficiency who developed an acute renal injury after an episode of hemoglobinuria. It was severe in one of the cases and the management required several sessions of dialysis, and on the other hand, the other case had a spontaneous remission after a properly conducted medical treatment. They were both known to be G6PD-deficient for 1 year in one case and 2 years in the other case, which was discovered following repeated hemolytic anaemia which required several ransfusions. However, these children were not followed up and no preventive measures had been taken by their parents. Although the G6PD deficiency had been established, the personnel not being trained on preventive measures did not sensitize the parents.

Discussion: This subject points out the importance of the continuous training of health personnel on the existence of this deficiency. G6PD deficiency, or favism is a deficiency of a red blood cell enzyme: Glucose-6-Phosphate Dehydrogenase. It is a sex-linked genetically inherited disorder, with a genetic trait of a normal child who will only be ill after being exposed to external 'oxidative' stress agents caused by ingestion of certain foods or drugs.

Conclusion: the prevention of exposition to these oxidants and the transmission of adequate information to the family and school entourage of these patients who are carriers of this deficiency as well as doctors is crucial

\section{Abstract 75:}

\section{CLINICAL PROFILE AND OUTCOME OF CHILDREN WHO RECEIVED RENAL REPLACEMENT THERAPY FOR ACUTE KIDNEY INJURY IN TIKUR ANBESSA SPECIALIZED HOSPITAL; AN ISN/ SRC TRIO EXPERIENCE}

Bezaye Abebe ${ }^{1}$, Damte Shimelis ${ }^{1}$, Priya Pais ${ }^{2}$, Arpana lyengar ${ }^{2}$

Addis Ababa University College of Health Sciences ${ }^{1}$, St John Medical College Hospital ${ }^{2}$.

Introduction: Acute kidney injury (AKI) requiring Renal Replacement Therapy (RRT) is associated with high mortality. The study assessed the impact of the introduction of RRT on outcomes of patients with AKI in Pediatric Nephrology unit in Tikur Anbessa Specialized Hospital (TASH) in Addis Ababa, Ethiopia. We provide acute Peritoneal Dialysis and acute intermittent Heamodialysis with support from the ISN SRC trio program for few selected cases due to lack of resources.

Methods: The study is a single center retrospective study that evaluated the clinical profile and survival outcomes of patients with AKI requiring Intermittent Hemodialysis and Acute Peritoneal Dialysis (IHD/ APD) at a tertiary hospital in Addis Ababa, Ethiopia. Data was collected on patients who received RRT for AKI from July 2016 to October 2018. Patient demographics, commodities, clinical presentation, laboratory tests, and mortality were reviewed and analyzed.

Results: There were 35 children in this cohort; median age was 9 years (IQR 4 months - 15 years). Females comprised $54.3 \%$ of the cohort. Infectious diseases including Acute Gastroenteritis, Pneumonia, and severe Sepsis 7 (20\%) and Acute Glomerulonephritis (AGN) 18 (54.28\%) were the most frequent commodities. Oligo-anuria with pulmonary edema (91.4\%) was the top indication for RRT. Overall mortality was $37.14 \%$. Underlying severe sepsis and pulmonary edema in AGN patients were the most common causes of death. Twenty one (60\%) of children had partial renal recovery on discharge. One child went home against medical advice. Eleven children received acute IHD in the adult Unit. Of the children who received APD, there was a $37.5 \%$ complication. Two children had catheter obstruction, 5 leaks from the insertion site and 2 children had peritonitis. We used a rigid catheters inserted at bedside with improvised fluids according to the ISPD guidelines using Ringer Lactate and Dextrose solutions.

Conclusions: The availability of acute RRT in Pediatric Nephrology unit in TASH has resulted in improved patient survival. Early detection of AKI and prompt protocol based management are essential in reducing the morbidity and 
mortality related to AKI. We recommend vigorous resuscitation and management of sepsis to save lives. The ISN SRC program helped our unit in providing physicians' training and equipment to deliver a standardized health care service to children with $\mathrm{AKI}$ in our institution.

\title{
Abstract 76:
}

\section{ACUTE KIDNEY INJURY FOLLOWING MULTIPLE BEE STING. A CASE REPORT}

Fareen Musa, Cyriaque Mbarubukeye ${ }^{2}$, Bashir Adman ${ }^{3}$

Paediatric Department University of Nairobi. Kenyatta National Hospital.

Background: Bee sting envenomation is rare pediatric emergency encountered worldwide, causing acute renal failure, multiple organ dysfunction and death. Allergic reaction can be triggered by a single sting and the greater the number of stings, the worse prognosis.

Case report: An eight year old boy was stung by a swarm of bees. He was referred to Kenyatta National Hospital for paediatrician nephrologist review at day 6 post bee sting. He received intravenous fluids and antihistamines for 2 days and 3 days in intensive care unit at provincial hospital where he had dark oliguria and dark urine, azotemia and elevated creatinine. Admission findings at Kenyatta National Hospital were generalized edema, hypotension of 60/40 $\mathrm{mmHg}$ and Glasgow coma scale of $11 / 15$. He received bolus and maintenance fluids, intravenous steroids, antihistamines and analgesics. The laboratory results revealed hemoglobin $9.7 \mathrm{~g} / \mathrm{dl}$ and thrombocytopenia of 105 , urea of $69.1 \mathrm{mml} / \mathrm{L}$, serum creatinine of $529 \mathrm{mmol} / \mathrm{L}$ with an estimated glomerular filtration rate of $12 \mathrm{ml} / \mathrm{min} / 1.73 \mathrm{~m}^{2}$, raised transaminases: AST 550, ALT 761. Peritoneal dialysis initiated within 24 hours. His consciousness level normalized after 2 days, his urine output after 3 days whereas liver and renal function normalized at day 7 and peritoneal dialysis was stopped. The child was discharged home at day 9 post admission.

Discussion: Bees sting usually results in pain and allergic reactions. Mass envenomation can cause systemic reactions and organ dysfunction including rhabdomyolysis, hemolysis, coagulopathy, hepatic, renal, cardiac and neurological complications. Rhabdomyolysis, hemolysis, direct nephrotoxic effect of envenomation and systemic vasodilation are distinguished cause of acute kidney injury.

Conclusion: Multiple wasp stings may cause acute kidney injury. Immediate supportive treatment including epinephrine, antihistamines and hydration is the mainstay to reduce morbidity and mortality in such cases.

\section{Abstract 77:}

\section{HEPATORENAL SYNDROME: A CASE REPORT OF ACUTE HEPATITIS A}

\author{
Cyriaque Mbarubukeye, Bashir Adman
}

East Africa Kidney Institute; Paediatric Department University of Nairobi. Kenyatta National Hospital.

Background: Hepatorenal syndrome (HRS) is the development of renal failure in patients with severe (acute or chronic) in the absence of any other identifiable cause of renal pathology.

Case report: A 8 year old male with 3 days history of yellowness of the eyes, fever, hematemesis, epistaxis, petechiae, purpura, dark reduced urine output was referred to paediatric emergency clinic of Kenyatta National Hospital for further management of acute liver failure secondary to Hepatitis A infection. His weight was $26 \mathrm{~kg}$, previous input was $2500 \mathrm{mls}$ and urine output was $300 \mathrm{mls}(0.48 \mathrm{mlsper} \mathrm{kg})$. The preliminary lab works in our facility showed, urea of $43.5 \mathrm{mmol} / \mathrm{L}$, creatinine of $1088 \mathrm{umol} / \mathrm{L}$, AST of $899 \mathrm{u} / \mathrm{L}$, ALT of $1230 \mathrm{u} / \mathrm{L}$ with total bilirubin of $800 \mathrm{u} / \mathrm{L}$ albumin of $25 \mathrm{~g} / \mathrm{L}$, INR of 2.4 and hemoglobin of $5 \mathrm{~g} / \mathrm{dL}$. The paediatrician gastroenterologist has been consulted and the child received intravenous metronidazole and ceftazidime, vitamin K, ranitidine oral nystatin, cholesteramine and multivitamins and has been transfused 4 times. The third day of admission the peritoneal dialysis was initiated and both kidney and liver function were improved after 10 days of peritoneal dialysis with urea of $2.9 \mathrm{mmol} / \mathrm{L}$, ceartinine of $35 \mathrm{umol} / \mathrm{L}, \mathrm{ALT}$ of $52 \mathrm{u} / \mathrm{L}$, AST of $8 \mathrm{u} / \mathrm{L}$, total bilirubin of $53 \mathrm{umol} / \mathrm{L}$ direct bilirubin of $7.7 \mathrm{umol} / \mathrm{L}$.

Discussion: The hepatorenal syndrome is diagnosed after exclusion of other causes of renal failure in patient with liver disease. It is characterized by impaired renal function and marked abnormalities in the arterial circulation and activities of endogenous vasoactive system. In kidney there is increased vasoconstriction that results in low glomerular filtration rate (GFR). In the extrarenal circulation especially in splanchnic, there is a predominance of arterial vasodilatation which result in reduction of the total or systemic resistance and arterial hypotension Conclusion: Hepatorenal syndrome is rare unique form of prerenal acute renal failure that frequently complicates acute liver failure and usually associated with poor outcome. Earlier intervention is critical for survival of the patients. 


\title{
Abstract 78:
}

\section{PAEDIATRIC KIDNEY TRANSPLANT IN KENYA: A SINGLE CENTRE EXPERIENCE}

Introduction: Kidney transplant is the preferred renal replacement therapy for patients with end stage kidney disease. However management of paediatric patients with kidney transplant in resource poor countries is evolving and groaning under several mental, financial and infrastructural challenges. The objective of the study is to evaluate the management of paediatric patients with kidney transplant in a kidney care Centre in Kenya.

Methods: This was a non-randomized prospective study. The study populations were post-transplant Paediatrics patients presenting between $1^{\text {st }}$ April t 2015 and $31^{\text {st }}$ April 2019. The biodata, pre and post-transplant details of these patients were documented. The data was analysed using SPSS Vs 17.

Results: A total of 17 Paediatric patients were studied with M: F ratio of 3:1, the mean age was $13.4 \pm 3.6$ years. Chronic glomerulonephritis, hypertension, diabetes mellitus and HIV related kidney disease were the commonest cause of CKD. Financial constraint delayed transplant in $66 \%$ and non-availability of donor in $17.2 \%$. About $76 \%$ of the transplants were in India and $81 \%$ either financed the transplant by NHIF directly or through a directly by parents relation. There was no cadaveric transplant and about $97 \%$ of the donors were related/parents. Tacrolimus, mycophenolate and prednisolone were most frequently used immunosuppressive combination. The one and three years graft survival were $98.3 \%$ and $87.6 \%$ respectively while corresponding patients survival were $97.7 \%$ and $92.4 \%$ respectively. Septicemia, acute rejection and urinary tract infection were most common complications. Conclusion: Management of patients with kidney transplant has good prospect despite the challenges.

\section{Abstract 79:}

\section{PREVALENCE AND FACTORS ASSOCIATED WITH THE OCCURRENCE OF MICROALBUMINURIA IN CHILDREN WITH SICKLE ANAEMIA IN TERTIARY HOSPITAL IN SOUTH-SOUTH NIGERIA.}

\author{
Enobong E. Ekpenyong \\ Paediatric Nephrology Unit, Department of Paediatrics, University of Uyo Teaching Hospital, Akwa Ibom State, Nigeria.
}

Objective: The prevalence of end stage renal disease (ESRD) in sickle cell anaemia (SCA) patients has been on the increase in the last few decades as the life expectancy of these patients has increased. The paucity of information about Sickle Cell Nephropathy (SCN) in sub-Saharan Africa which has the highest burden of SCA, may play a role. In addition, the use of microalbuminuria in evaluation of SCA patients has not received sufficient attention particularly in Nigeria. In this study, the aim was to determine the prevalence and factors associated with microalbuminuria in children with sickle cell anaemia aged 1-17 years.

Methods: One hundred and two known HbSS children aged 1-17yrears in steady state were recruited into a prospective cross-sectional study. Demographic and clinical findings were recorded. Albuminuria was assayed using spot urine using the immunuturbidimetric method. Urine creatinine concentration was estimated using the Roche Reflotron test strips for quantitative determination of creatinine in blood, serum, plasma and urine. Albumin to creatinine ratio was then calculated. Microalbuminuria was defined as Albumin Creatinine Ratio (ACR) of 30-300mg/g. Results: The results showed that the prevalence rate of microalbuminuria in these patients was $23.5 \%$. There was an increasing prevalence as the age of the subjects increased, however it was detected in children as young as three years of age. Age, gender, packed cell volume showed a significant relationship with the occurrence of microalbuminuria in this study. On further analysis, increasing age, female gender and lower packed cell volume emerged as independent risk factors associated with the occurrence of microalbuminuria in the study population. Conclusion: From the findings, microalbuminuria occurs with a high prevalence in children with sickle cell anaemia. Early routine screening is recommended to identify patients with renal damage for proper management to prevent progression to ESRD. 


\section{Abstract 80:}

\section{A RETROSPECTIVE STUDY ON THE OUTCOMES OF PERITONEAL DIALYSIS AMONG PEDIATRIC PATIENTS WITH ACUTE KIDNEY INJURY AT THE KENYATTA NATIONAL HOSPITAL}

Background: Epidemiological evidence supports the view that acute kidney injury (AKI) has some adverse clinical consequences. The dialytic interventions for infants and children take different forms, peritoneal dialysis (PD) has been preferred because of its efficacy.

Aim: To establish the outcomes of PD on pediatric patients in Kenyatta National Hospital (KNH).

Methodology: The study design was a retrospective cohort study. The study population was pediatric patients managed on PD due to AKI for the period, January 2017 to December 2017. The sample size was 102 files calculated using Yamene Taros formula. Census sampling method was used. Data was collected using a structured checklist, entered, cleaned and analyzed using SPSS. Paired t-test was used to determine biochemistry parameter before commencement and on discontinuation of PD. The statistical significance was set up at $p<0.005$.

Results: The survival rate was $58.8 \%$ and mortality rate $41.2 \%$. None of the participants progressed to chronic renal disease. The mean Serum Creatinine was higher (602) prior to PD and lower on discontinuation (243). Serum urea level was higher prior to PD and lower on discontinuation of PD (51.49>14.58).

Conclusion: A higher percentage of the participants had successful restoration of kidney functions however the mortality rate at $41.2 \%$ is still a high percentage. This is an indicator that PD is an effective modality in reduction of elevated serum urea and creatinine in treatment of AKI in pediatric patients.

Recommendations: There is need to avail affordable PD services at the county hospitals to enhance easy accessibility of this service. There is need to carry out a prospective study in pediatric patients on PD due to AKI to determine the outcomes after PD therapy.

\section{Abstract 81:}

\section{URGENT START DIALYSIS: COMPARISON OF PERITONEAL DIALYSIS VERSUS HEMODIALYSIS}

Neelam M. Bhalla ${ }^{1 *}$, Neiha Arora ${ }^{1}$, Jeanne A. Darbinian ${ }^{1}$, and Sijie Zheng ${ }^{1}$

${ }^{1}$ Kaiser Permanente, Northern California.

Background: Though underutilized in the US, PD is a safe and effective modality of renal replacement therapy. One reason for under use of PD is the practice of initiating patients on HD via a central venous catheter (CVC) if they do not have a functional AVF/AVG. When compared to AVF/AVG, CVC use is associated with increased mortality. Recently, use of urgent start PD has gained considerable interest since it decreases use of CVCs, while affording a mechanism of increased PD utilization. In this retrospective cohort study, we compared complications and outcomes between the two urgent start dialysis modalities.

Methods: We identified a subpopulation of KPNC members who met clinical criteria for being in urgent need of beginning PD or HD between $1 / 1 / 2011$ and 12/31/2014. Urgent start HD patients were matched 3:1 with urgent PD starts on selected characteristics. Medical records of all cohort members were reviewed by three nephrologists. Complications and outcomes occurring after initiation of dialysis were compared between them using Chi-square or Fisher exact tests.

Results: We compared 335 HD starts with 84 PD starts. There were no modality switches in the PD cohort; in HD start cases one changed to home HD and four to PD. Transferring to hospice accounted for $92 \%$ of modality terminations in HD; reasons in PD were psychosocial (43\%); medical (28.6\%); peritonitis (14.3\%) and others (14.3\%). Major complications were low in both groups $(<5 \%)$, though rate of catheter malfunction was higher in $\mathrm{HD}(12.8 \%$ vs. $6.0 \%$, $\mathrm{p}=0.09)$. There was a statistically significant difference in overall infectious complications between PD (20\%: peritonitis $-13 \%$; exit site - 7\%) and HD (9\%: bacteremia - $7.2 \%$; exit site - 1.8\%, $\mathrm{p}<0.01)$. There were more deaths in the HD group $(19.7 \%$ vs $7 \%, p=0.015)$. Furthermore, a higher proportion of deaths were observed in patients with bacteremia, compared with those who had peritonitis (25 vs. 16.7\%). Pericatheter leaks developed in $7 \%$ of PD cases and in no HD patients.

Conclusion: Urgent PD start is a viable alternative to urgent HD start via CVC. Although infectious complications were higher in PD, peritonitis was associated with less mortality than bacteremia. Notably, there is a high patient retention rate, leading to increased utilization of PD. 


\section{Abstract 82:}

\section{CAUSES OF STOPPING PERITONEAL DIALYSIS}

\section{PD Churn}

Background: Peritoneal Dialysis (PD) is an excellent option for renal replacement therapy (RRT). Kaiser Permanente Northern California (KPNC) is an integrated health care system with around 4 million members and 4700 patients on dialysis. Our PD prevalence is higher (24\%) compared with the national average (8\%). We retrospectively analyzed the reasons for stopping PD.

Methods: Retrospective analysis of all patients who were on PD at any time from 10/1/2013 to 12/21/2014. They were followed through $3 / 31 / 2015$. We identified patients who stopped peritoneal dialysis for at least 60 -days. We excluded patients who lost KP membership while active on PD and included patients who stopped PD due to a kidney / SPK transplant.

Results: We identified 502 PD patients in the study period. Death (29\%) and Transplantation (23\%) were the two main reasons for stopping PD. Other reasons include: 1. Psycho social (12\%); 2. Peritonitis (11\%); 3. Non-PD related medical issues (8\%); 4. Peritoneal membrane failure (7\%); 5 . Mechanical complications of PD (5\%); 6 . Peritoneal Catheter Leaks (2\%); 7. Other $(<1 \%)$. The average time on PD was 2.01 year (Median of 1.36 years; range: 0.02 to 17.59 years).

Conclusion: Besides death and transplant, psychosocial issues and peritonitis were top potentially preventable reasons for patients to stop PD in our analysis. Further analysis is needed to analyze primary causes of death and strategies to prevent peritonitis and provide psychosocial support to patients to remain on PD.

\section{Abstract 83:}

\section{KIDNEY DONATION AND TRANSPLANT IN BENIN: UNIVERSITY OF ABOMEY-CALAVI STUDENTS PERCEPTION}

Agboton Bruno Leopold ${ }^{1}$, Hazoume Rodrigue ${ }^{1}$, Vigan Jacques ${ }^{1}$, Ahoui Seraphin ${ }^{2}$, Azonbakin Simon ${ }^{3}$, Elhadji Fary Ka ${ }^{4}$, Laleye Anatole ${ }^{3}$ Bigot André ${ }^{5}$

1 Nephrology - Dialysis Healthcare Center, CNHU HKM Cotonou, Benin; 2 Nephrology -Dialysis, Parakou Teaching Hospital, Benin; 3 Cytogenetics and Medical Genetics, Histology and Reproductive Biology Laboratory, Human Biology Unit, Faculty of Health Science, Cotonou; 4 Nephrology Department, Aristide le Dantec Université Cheikh Anta Diop Dakar Sénégal ; 5 Biochemistry Laboratory CNHU-HKM - Pharmacy / University of Abomey Calavi.

Objective: Analyze University of Abomey-Calavi students perception about kidney donation and transplant. Methods: It was a descriptive and analytical cross-sectional study conducted in four (4) higher education centers from 15 th May to 17 th August 2017. All students who met the inclusion criteria and gave their informed consent were included in the study. Data were collected with a sheet designed on the basis of different variables and processed by Excel 2010 and SPSS 21.

Outcomes: The study covered four hundred and one students, with a sex ratio $=1.25$. The mean age was $21.68 \pm 2.32$ years. The majority of respondents were medical students (77.6\%). They had good knowledge of diseases requiring transplant $(85.8 \%)$, and $95.26 \%$ of them were familiar with the transplantable organs. Over half of the respondents (77.3\%) agreed to donate their kidney after death. For two-thirds $(70.32 \%)$ of the students included in the study, there is need for public awareness in view of the successful implementation of such activity. The grounds for refusal to donate their kidney were as follows: religion $(p=0.35)$ ethnicity $(p=0.035)$, infringement of bodily integrity $(59.6 \%)$. Among the students who know a kidney transplant recipient, 75\% agreed to donate their kidneys after death ( $p=$ 0.018).

Discussion and Conclusion: The study indicates that the University of Abomey-Calavi (UAC) students have limited knowledge about kidney donation. Some factors influencing student choice include religion, ethnicity and bodily integrity. Development of transplant in Benin requires regular awareness-raising and motivation of the general population. 


\section{Abstract 84:}

\section{A RARE CAUSE OF SEVERE ANEMIA FOLLOWING KIDNEY TRANSPLANTATION}

Momina M.Ahmed ${ }^{1}$ M.D, Afework Hagos ${ }^{1}$ M.D.,Mesfin Asefa ${ }^{1}$ M.D.,Hamelmal Gebeyehu ${ }^{1}$ M.D., Mamo Negussie ${ }^{1}$ M.D.,Fasika Tedla ${ }^{2}$, M.D., Mersema Abate ${ }^{3}$, M.D .,Berhanu Worku M.D. , Seyfemichael Getachew ${ }^{1}$ M.D.,, Leja Hamzaํ․․ . Lina Mohamed ${ }^{1}$ M.D., , Aklilu Yishak ${ }^{5}$ M.D. , Mahteme Bekele ${ }^{1}$ M.D., Engida Abebe ${ }^{1}$ M.D., Mekdim Tadesse $^{1}$ M.D., Tekleberhan Berhe ${ }^{1}$ M.D., Kenneth J Woodside ${ }^{4}$, M.D., Alan B. Leichtman ${ }^{4}$ M.D., Wubishet Jote ${ }^{1}$ M.D. Jeffrey D Punch ${ }^{4}$ M.D.

1Saint Paul's Hospital Millennium Medical College, Addis Ababa, Ethiopia; 2SUNY Downstate Medical Center, Brooklyn, New York, USA; 3Stony Brook University Hospital, Stony Brook, New York, USA; 4 University of Michigan, Ann Arbor, Michigan, USA; 5 Mc Lean, Virginia, Inova Alexandria Hospital, USA.

Introduction: Anemia is common in post kidney transplant patient due to multifactorial cause. In a well-functioning graft anemia usually resolve with few months. Here we describe a rare cause of severe anemia following kidney transplant.

Case: A 20 year old young gentleman came in with end stage renal failure with uremia of unknown cause. After two years of dialysis he received a live donor kidney transplant. He received induced Simulect for induction and maintained on tacrolimus, mycophenolate mofetil and prednisolone .Post transplant course was uneventful .A month later he was noted to have decline in hemoglobin. Despite erythropetin therapy it progressively declined , reached to its nadir $4.8 \mathrm{gm} / \mathrm{dl}$ a month later ( Fig .1)). He required few units of blood transfusion. In the meantime tests for Hemolysis markers, substrate deficiency (Iron, Vitamin B12 and folate) were all negative. Bone marrow aspiration demonstrated erythroid hypoplasia with occasional giant proerythroblast which is suspicious of parvo B19. Though serology both IgG and IgM were negative, qualitative PCR were positive for Parvo B19 Virus. Four doses of intravenous immunoglobulin administered besides reduction of immunosuppression. Within two weeks' time the anemia remarkably sustained improvement. Allograft function was stable throughout.

Discussion: Anemia is well-recognized complication post kidney transplantation due to multifactorial etiology. Parvo infection is a rare but significant cause severe anemia in immunosuppressed patients such as kidney transplant patient. Serology tests might be deceiving in immunosuppressed patients to diagnose the infection due to inadequate humoral response as in our case. Detection of viral DNA by PCR, though it qualitative with the clinical setting and suggestive BM aspiration likely to be active infection.

Conclusion: Parvo B19 though rare is an important treatable cause of severe anemia after kidney transplant. There should be high index of suspicion of Parvo B19 infection in severe anemia following kidney transplant.

\section{Abstract 85:}

\section{LIVING DONOR KIDNEY TRANSPLANT: ETHIOPIAN EXPERIENCE}

Momina M.Ahmed ${ }^{1}$ M.D,Hamelmal Gebeyehu¹ M.D., Mamo Negussie ${ }^{1}$ M.D.,Fasika Tedla², M.D., Mersema Abate ${ }^{3}$,

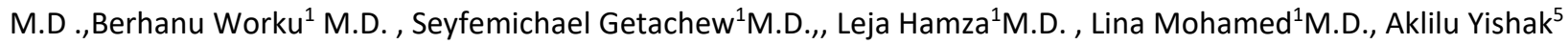
M.D. , Mahteme Bekele ${ }^{1}$ M.D., Engida Abebe ${ }^{1}$ M.D., Mekdim Tadesse ${ }^{1}$ M.D., Tekleberhan Berhe ${ }^{1}$ M.D., Kenneth J Woodside ${ }^{4}$, M.D., Alan B. Leichtman ${ }^{4}$ M.D., Wubishet Jote ${ }^{1}$ M.D. Jeffrey D Punch ${ }^{4}$ M.D.

1Saint Paul's Hospital Millennium Medical College, Addis Ababa, Ethiopia; 2SUNY Downstate Medical Center, Brooklyn, New York, USA; 3Stony Brook University Hospital, Stony Brook, New York, USA; 4University of Michigan, Ann Arbor, Michigan, USA; 5 Mc Lean, Virginia, Inova Alexandria Hospital, USA.

Background: Kidney transplant is the most cost effective way to treat end stage renal failure. Ethiopia as one of resource limited Country launched "living donor kidney transplant first "program in Addis Ababa at St Paul's Hospital Millennium Medical College in Sept 2015. Dialysis provided whenever necessary for short period as a bridge to kidney transplant. Outcomes of donor and recipients are the parameters to assess the center performance. This study reports on live donor and recipient outcomes to date.

Methodology: A retrospective review and analysis of medical records of all live donor and recipients who participated in the program from Sept 2015 through May 2019.

Results: A total of 114 kidney transplants performed. Male to Female ratio for recipient and donors were 2.5:1 and 1:1 respectively. The mean age for the recipients were 34.2 while for the donors were 35.1 . Sibling constitute $53 \%$ the donors followed by parents, offspring, spouses and cousins in order. There was no major complication on the donors except one patient who required laparotomy for small bowel obstruction. The etiology of renal failure was unknown in $72 \%$ of cases. Half of the patients had history of transfusion. The first 42 kidneys recovered by hand assisted 
laparoscopic nephrectomy then due to shortage of supply shifted to open nephrectomy. All except one received induction therapy with basiliximab. Most used combination immunosuppressive therapy were tacrolimus, mycophenolate and prednisolone. There were two primary graft failure on the table and Seven deaths. The cause of death in all cases were infection. Overall graft and patient survival is $91 \%$ and $94 \%$ respectively. Most common Post transplant complications were erythrocytosis (25\%), Infection (21\%), diarrhea (15\%) and post-transplant diabetes (7\%).

Conclusion: The outcomes of St. Paul's kidney transplant program are comparable to the international standards. It's a good testimonial that in a resource limited setting where there is limited dialysis service living donor kidney transplant is a viable option to treat renal failure.

\title{
Abstract 86:
}

\section{FACTORS ASSOCIATED WITH UNCONTROLLED HYPERTENSION AMONG RENAL TRANSPLANT RECIPIENTS ATTENDING NEPHROLOGY CLINICS IN NAIROBI, KENYA}

Kubo $\mathrm{M}^{*}$, Kayima J, Were A, McLigeyo S, Ogola E

Objective: To determine the factors associated with poor blood pressure control among renal transplant recipients in a resource limited setting.

Methods: A cross-sectional study was carried out on renal transplant recipients at the Kenyatta National Hospital. Sociodemographic details, blood pressure, urine albumin : creatinine ratio, and adherence using the MMAS- 8 questionnaire were noted. Independent factors associated with uncontrolled hypertension were determined using logistic regression analysis.

Results: 85 subjects were evaluated. Mean age was 42.4 (SD \pm 12.2 ) years, with a male : female ratio of $1.9: 1$. Fiftyfive patients $(64.7 \%$ ) had uncontrolled hypertension (BP $\geq 130 / 80 \mathrm{mmHg}$ ). On univariate analysis, male sex (OR 3.7, $95 \% \mathrm{Cl} 1.4-9.5, p=0.006)$, higher levels of proteinuria $(p=0.042)$, and nonadherence to antihypertensives (OR 18, $95 \% \mathrm{Cl} 5.2-65.7, p<0.001)$ were associated with uncontrolled hypertension. On logistic regression analysis, male sex (adjusted OR 4.6, 95\% Cl 1.1-19.0, $p=0.034$ ) and nonadherence (adjusted OR 33.8, 95\% Cl 8.6-73.0, $p<0.001$ ) were independently associated with uncontrolled hypertension.

Conclusion: Factors associated with poor blood pressure control in this cohort were male sex and nonadherence to antihypertensives. Emphasis on adherence to antihypertensive therapy must be pursued within this population.

\section{Abstract 87: \\ CHANGES IN BLOOD PRESSURE LEVELS AND ANTIHYPERTENSIVE MEDICATION USE PRE- AND POST RENAL TRANSPLANTATION AMONG PATIENTS IN NAIROBI, KENYA: A COMPARATIVE CROSS-SECTIONAL STUDY}

\author{
Kubo $M^{*}$, Kayima J, Were A, McLigeyo S, Ogola E
}

Objectives: To determine the changes in blood pressure levels and antihypertensive medication use in the post renal transplantation period compared to pre-transplantation.

Methods: A comparative cross-sectional study was carried out on renal transplant recipients at the Kenyatta National Hospital, a national referral hospital in Kenya. Sociodemographic details, blood pressure levels and antihypertensive medication use pre-and post renal transplantation were noted. Changes in mean blood pressure levels and mean number of antihypertensive medications post renal transplantation were determined using paired t test.

Results: 85 subjects were evaluated. Mean age was 42.4 (SD \pm 12.2 ) years, with a male : female ratio of $1.9: 1$. Compared to the pre-transplant period, significantly lower mean systolic and diastolic blood pressure levels posttransplantation were noted(mean SBP $144.5 \mathrm{mmHg}$ vs $131.8 \mathrm{mmHg}$; mean DBP $103.6 \mathrm{mmHg}$ vs $83.5 \mathrm{mmHg}$ in the preand posttransplant periods respectively, $p<0.001)$. Mean number of antihypertensive medications also reduced significantly post transplantation, with an average of $3.3( \pm 1.6)$ vs $2.1( \pm 0.9)$ in the pre- and post transplant periods respectively $(p<0.001)$.

Conclusion: There is a significant reduction in blood pressure levels and number of antihypertensive medications used post renal transplantation. The positive impact of renal transplantation on blood pressure control should be confirmed using prospective cohort studies of patients with end stage renal disease who then undergo renal transplantation. 


\title{
Abstract 88:
}

\section{ADHERENCE TO IMMUNOSUPPRESSIVE THERAPY AMONG RENAL TRANSPLANT RECIPIENTS AT KENYATTA NATIONAL HOSPITAL}

\author{
R.C Bore, J.K Kayima, M.K Joshi, M.C Maritim
}

Background: Renal transplant is the treatment of choice in end stage renal disease. Key in maintaining graft survival is use of immunosuppressive therapy as prescribed by the KIDIGO guidelines

Aim: The primary objectives were to determine the proportion of kidney transplant recipients (KTRs) adherent to immunosuppressant therapy and the relationship between adherence and graft function. The secondary objective was to identify the factors barring adherence to immunosuppressant therapy (IST).

Setting and Design: A cross-sectional descriptive study carried out at the Kenyatta National Hospital (KNH).

Methods: The KTR was weighed in kilograms and a blood sample was then taken for serum creatinine level to calculate the estimated glomerular filtration rate (eGFR) using the Cockcroft-Gault formula as a marker of graft function. Simplified Medication Adherence Questionnaire (SMAQ) was filled in by the patient or caregiver to assess for adherence. Statistical analysis was performed in SPSS version 21.0 software. Proportion of adherence to immunosuppressants was presented as percentages with $95 \%$ confidence intervals (Cls). Factors influencing adherence to immunosuppressants was tested using chi square test for categorical variables and independent $t$ test to compare means. A multivariate analysis was done to assess for factors independently associated with adherence.

Results: A total of 106 KTRs were recruited between January and April 2018. $70.8 \%$ of kidney transplant recipients were adherent and there was a positive relationship between adherence and graft function with adherent KTRs having a median eGFR of $79 \mathrm{~mL} / \mathrm{mL} / \mathrm{min} / 1.73 \mathrm{~m}^{2}$ whereas non-adherent $\mathrm{KTRs}$ had a median eGFR of $42 \mathrm{~mL} / \mathrm{min} / 1.73 \mathrm{~m}^{2}$ and this was statistically significant with a $p$-value of $<0.001$. Factors barring adherence to immunosuppressive therapy included: KTR young age at transplantation, employment status, shorter duration of dialysis pretransplantation and pill count.

Conclusion: Adherence in our population was comparable to other centers in the world at $70.8 \%$ but we still have room for improvement of adherence addressing factors identified.

\section{Abstract 89:}

\section{KIDNEY TRANSPLANT IN HIV INFECTED PATIENTS - OUR EXPERIENCE AND LITERATURE REVIEW}

Sanand Bag, A Srinivas Murthy

Renal Transplant Unit - Mediheal Hospitals \& Fertility Centre, Eldoret, Kenya.

Objectives: Due to effective anti-retroviral (HAART) kidney transplantation, once contraindicated, has become better alternative to dialysis for HIV positive ESRD patients. We present the first successful kidney transplant on HIV positive recipients in Kenya, and discuss experience of 5 such cases, with key issues in management.

Methods: We performed the first successful renal transplant in HIV patient in Kenya in April 2019. Data of 4 HIV patients successfully transplanted earlier by our team in India are compiled. ESRD patients requiring dialysis, on HAART, in good health, viral-RNA negative $(\leq 50)$, CD4 Lymphocytes $>200$ were selected. All transplanted from living related donors. Standard immunosuppression protocol used (ART stopped during peri-op period) without induction except the last patient (Thymoglobulin).

Results: Previous 4 patients had uneventful post-operative recovery - discharged within 10 days with normal creatinine (mean 1.32). Last patient had many challenges and stormy post-operative course with AbMR (Class-II DSA+) that responded to Plasma-Exchange + IVIg. All recipients are well on follow up (mean Creat 1.27; no viremia). Conclusions: Although some studies show higher graft rejection, kidney transplant in selected HIV-patients is preferable over dialysis (increased survival, quality life, no impact on HIV- evolution). Multidisciplinary managements, careful monitoring needed - interactions of immunosuppression and anti-retroviral medications may lead to overwhelming viremia on one end; increased rejection, nephrotoxicity on other-end. Mycophenolate additionally controls HIV proliferation. Ritonavir (Cyp450-inhibitor) reduces tacrolimus level - judicious increase dosage (keeping eye on nephrotoxicity). Lamivudin, Abacavir, Tenofovir, Valtecavir are safe - no significant drug interactions. 


\section{Abstract 90:}

\section{LAPAROSCOPIC DONOR NEPHRECTOMY: STANDARD OF CARE AND MOTIVATING}

Sananda Bag, A Srinivas Murthy

Kidney Transplant Unit - Mediheal Hospitals \& Fertility Center: Eldoret, Kenya.

Objectives: Kidney transplant is best treatment for chronic kidney failure, mainly from living donors. Donors get operated for someone's benefit - should get minimal pain, early recovery, no complication or long-term sore. Laparoscopic donor nephrectomy (LDN) imparts all benefits. We present LDN outcomes from our initial series of renal transplants.

Methods: Prospective acquisition of donors' \& recipients' data for all patients in our kidney transplant unit done from Dec2018-till date. After detail evaluation, requisite clearances, consent (conversion to open) LDN were performed in all donors. Dissection was carried out through standard 3-4 ports technique, kidney extracted through small suprapubic (non-muscle-cutting) incision. Multiple arteries are anastomosed to make common lumen to be anastomosed to internal or external iliac artery of recipient.

Results: 30 successful renal transplants are performed till date (abstract submission), with LDN in 29 cases from family / related donors. Donor's mean age was 33.96+8.89yrs; male:female 18:11; BMI 21.6+3.9. Left LDN successfully done in all. Mean operating time was $93.7 \pm 12.6 \mathrm{~min}$; Lap dissection $52 \pm 15 \mathrm{~min}$; warm ischemia $5.5 \pm 1.4 \mathrm{~min}$, allograft weight $143+19.5 \mathrm{gm}$. Ureter, vessels length was adequate; accessory renal arteries (5), duplex ureters (1) were managed properly. No surgical complications noted in donors or recipients. Donors had overall less pain, ambulated in 24 hours, discharged by 72 hours. Immediate diuresis, normal allograft function was achieved in all recipients but 4 , who had delayed (ATN) but complete recovery.

Conclusions: Laparoscopic donor nephrectomy should be standard of care - as it benefits the donors, is motivating without affecting graft function.

\section{Abstract 91:}

\section{KNOWLEDGE AND ATTITUDE OF UNDERGRADUATE MEDICAL STUDENTS IN KENYA TOWARDS SOLID ORGAN DONATION AND TRANSPLANTATION}

Mongare Newnex Brian, Mpekethu Nelson Mweteri, Marie-Claire Wangari, Daniel Ojuka Department of Surgery, College of Health Sciences, University of Nairobi, Kenya.

Objective: To describe the knowledge and attitudes of Kenyan undergraduate medical students towards solid organ donation and transplantation, and their level of competence in handling end-of-life issues.

Methods: A sample of undergraduate medical students from all the medical schools in Kenya offering a Bachelor of Medicine and Bachelor of Surgery (MBChB) were prospectively surveyed using a self-administered web-based questionnaire, between July and September 2018.

Results: There were 303 participants; 167 (55.1\%) were female and 136 (44.9\%) were male. Only $8.9 \%$ of the students had read the laws governing organ donation and transplantation in Kenya. An even lower percentage (3.3\%) felt that they had learnt enough about solid organ donation from their $\mathrm{MBChB}$ curriculum. $52.8 \%$ of the respondents would subscribe as solid organ donors; which reduced to $46.5 \%$ when it came to consenting the donation of their relative's organs. Less than half of the students felt they would be comfortable introducing the topic (39.6\%) or confident answering questions (22.8\%) related to organ donation and transplantation. Only $9.9 \%$ of the students had ever spoken to a patient about organ donation. There was no significant association between level of study (pre-clinical versus clinical) and comfort introducing the topic of organ donation ( $p=.206)$ or experience talking to a patient about organ donation $(p=.102)$.

Conclusion: Undergraduate medical students have significant knowledge gaps regarding organ donation and transplantation and feel ill-prepared to approach a potential donor or transplant recipient. Introduction of dedicated organ donation education programs targeting medical students can be used to address these deficits. 


\section{Abstract 92: \\ A COMPREHENSIVE REVIEW OF 30 CASES OF KIDNEY TRANSPLANTS PERFORMED OVER 6 MONTHS: A SINGLE CENTRE EXPERIENCE}

Murthy AS, Bag S.

Mediheal group of hospitals, Eldoret, Kenya.

A series of 30 kidney transplants were performed over a period of 6 months (Dec 2018- May 2019) at a private hospital successfully. All the cases were live donor kidney transplants with majority of the donors being $1^{\text {st }}$ degree relatives or spousal donors. There were no cases of mortality and all the patients had good immediate graft function except in 3 patients, who had delayed graft function; 2 of them improving over 2 weeks and another, still dialysis dependent [ 10 days post op at the time of submission of this abstract]. Renal allograft biopsy was done in two patients. One was suggestive of ATN, the other, report awaited at the time of submission of this abstract. Of all patients, 2 cases were HBsAg +ve, and one patient was HIV +Ve. Two patients required simultaneous nephrectomy at the time of transplant, one for ADPKD and the other for VUR. Other postoperative problems included; one case of sub acute intestinal obstruction due to adhesions requiring laparoscopic intervention and the other segmental consolidation with cough and fever. All the patients received induction with rabbit Thymoglobulin, and IV methyl prednisolone. 4 patients required plasma exchange and IVIG for + Ve X match $\{3$ pre op' and 1 post op'\}. Overall patient survival was $100 \%$ and graft survival over $98 \%\{29 / 30\}$.

\section{Abstract 93:}

\section{FACTORS INFLUENCING RELUCTANCE IN KIDNEY TRANSPLANTATION IN END STAGE RENAL DISEASE PATIENTS IN NAIROBI KENYA.}

\section{Kimani P}

Introduction: Recent studies confirm that the global burden of CKD is high with a meta-analysis of 44 country prevalence studies estimated the worldwide prevalence of CKD at $13.4 \%$.

Objectives: To determine the demographic and psychosocial variables associated with reluctance towards kidney transplantation as a form of renal replacement therapy

Methods: 46 hemodialysis patients from a single centre in Nairobi city and were recruited using consecutive sampling technique. Inclusion criteria was all patients between the ages of 18-65 years. The entire hemodialysis population was included. A 31 question questionnaire was developed for this study.

Results: 20 females and 26 males were included between the ages of 18-65 years. Reasons given by the three actively pursuing a transplant were be freedom to travel and work and persuasion by doctors and family members. For the 25 not actively pursuing a transplant, 8 did not have donors, 13 did not have adequate finances for transplant and follow up, 4 were worried about rejection and going back to dialysis.

For the 18 not interested in transplant 3 cited cultural (spiritual) factors, 6 hoped that their kidneys would start functioning again and 9 said dialysis was working fine for them. 2 patients would have considered transplantation if the donor was unknown to them.

Limitations: Major limitation of this study was sample size; the small number of participants in each group made it impossible to achieve statistical significance and thus larger studies are warranted before generalizations can be made. Also there is concern on representation of study sample since while it may appear typical, only participants from one facility in one geographic location were used.

Conclusion: Despite evidence of the benefits of transplantation over hemodialysis, a significant number of patients eligible for kidney transplantation are choosing dialysis due to financial difficulties, lack of organ donors and misconceptions on their disease. While further research in this area is warranted, these trends suggest the need for health education for hemodialysis patients and further research into the economic benefits of transplantation over hemodialysis in the Kenyan Setting. 


\title{
Abstract 94: \\ IMPACT OF KIDNEY DONATION ON QUALITY OF LIFE OF KIDNEY DONORS
}

Nyarera DK.

Background: End stage renal disease (ESRD) has become an important contributor of morbidity and mortality among patients with non-communicable diseases globally. Renal transplantation is well established as the best treatment for restoring quality of life to these patients, however getting a willing and suitable donor has remained the major challenge. Living donor kidney transplantation (LDKT) is reported to have better graft survival and overall patient outcomes compared to deceased donation. The prospective donors also have a legitimate right to know the quality of life of existing donors. Some prospective donors still having fear of unknown and are not sure of what their life will be I after kidney donation. The aim of this study was to assess impact of kidney donation on quality of life of donors.

Methodology: Data was collected from all participants using a questionnaire to help evaluate their socio demographic and socio economic status pre and post kidney donation.

Results: During the study period, 84 Living Donors who had undergone donor nephrectomy for kidney donation between January 2010 and September 2014 were interviewed. 48 [57.1\%] were male and 36 [42.9\%] were female. $64.3 \%$ of the donors were discharged from the hospital by the $5^{\text {th }}$ day after surgery and $58.3 \%$ resumed their normal duties before 2 months.84.5\% maintained their previous occupation and $15.5 \%$ changed their occupation with reasons of better paying job than what they were doing previously. $97.6 \%$ reported no hospitalization after kidney donation compared with $78.6 \%$ before kidney donation. $84.5 \%$ of the respondents reported no change in their social life after kidney donation. $97.6 \%$ could recommend somebody to donate a kidney, $94 \%$ reporting that it saves life and that their lives have not changed, they have been able to go on with their life normally. Ninety-eight percent (98\%) of Living Donors believed that their quality of life did not decrease after Living Kidney Donation.

Conclusion: Kidney donation does not have a significant negative impact on quality of life of kidney donors since even after donation the donors remain physically and mentally healthy as indicated by $84.5 \%$ of the donors. All the donors maintain their social life and report the experience to be rewarding and satisfying since they can save a life and increase their self-esteem. They also reported kidney donation to have changed their live for better because they are more disciplined and more conscious of their lifestyle.

\section{Abstract 95:}

\section{EFFECTS OF CLASSROOM EDUCATION ON KNOWLEDGE AND ATTITUDES REGARDING ORGAN DONATION AMONG MOROCCAN MIDDLE AND HIGH SCHOOL STUDENTS}

\author{
Elassas H,Chettati M, Fadili W, Laouad I \\ Nephrology-Hemodialysis departement, Cadi Ayyad university, CHU Mohammed VI, Marrakech, Morocco.
}

Introduction: Adolescents are an important target group for organ donation education. As yet, little is known about the attitude of Moroccan adolescents toward deceased organ donation, or the factors related to their decision. In this study, we explored the knowledge and opinions of deceased organ donation among middle and high school students in Morocco.

Methods: A self-reported survey was conducted among 810 middle and high school students in Marrakech, Morocco. The questionnaire assessed knowledge, personal experience, and opinions of organ donation. Logistic regression analysis was performed to determine significant factors associated with willingness to become an organ donor. The impact of a classroom intervention was studied. A baseline survey was administered to all classes within 2 weeks prior to intervention. On the intervention day, classes randomly assigned to the intervention group received an educational session, followed by a second survey; in control classes, the second survey was taken before the educational session. In the demographic analyses, the Student's $t$-test was used for continuous variables, with values of $p<0.05$ considered significant, and Pearson's chi square statistic for categorical variables. All analyses were performed using SPSS21.0 .

Results: Of the 810 survey respondents, $41.8 \%$ were females, $58.2 \%$ were males. Mean age of the sample was $14 \pm$ 2.7. Of the 810 students, 558 (69.8\%) were willing to be a deceased donor, and the other 252 (30.2\%) were unwilling or unsure. Religious belief, socioeconomic status and family discussion were strong predictors of the willingness of Moroccan middle and high school students to be an organ donor.

Following the intervention, students in the intervention group demonstrated a significant increase in knowledge scores $(p<0.001)$, as well as positive movement of opinion regarding willingness to donate $(p<0.0001)$. Most importantly, the positive changes in opinion occurred independently of socioeconomis status and religious belief, in 
spite of these both being negative predictors of opinion at baseline. These results demonstrate that even a single classroom exposure can impact knowledge levels, correct misinformation, and effect opinion change on organ donation among adolescent population.

Conclusions: A donation education program that promotes family communication and discussion about organ donation in school could increase willingness to be an organ donor among Moroccan adolescents.

\title{
Abstract 96:
}

\section{EVALUATION OF POTENTIAL LIVING DONORS FOR KIDNEY TRANSPLANTATION AT A TERTIARY HEALTH INSTITUTION IN SOUTH-EAST NIGERIA- A PRELIMINARY REPORT}

\author{
Okwuonu $\mathrm{CG}^{1}$, Ukpabi OJ ${ }^{1}$, Achor $\mathrm{G}^{2}$, Erondu N ${ }^{3}$, Aguocha $\mathrm{G}^{4}$, Akoma $\mathrm{C}^{5}$, Kanu $\mathrm{H}^{5}$, Chuku $\mathrm{A}^{6}$, Ekwenna $\mathrm{O}^{7}$ \\ 1Department of Internal Medicine, 2Department of Surgery, 3Department of Anaesthesia, 4.Department of Psychiatry, \\ 5Nursing Services Division, 6Department of Ophthalmology, Federal Medical Centre Umuahia Abia State Nigeria, \\ 7Division of Urology, University of Toledo Medical Centre Toledo Ohio USA.
}

Introduction: Kidney transplantation is developing in Nigeria. Availability of suitable, willing, living donors is one of the major factors leading to its success. The clinical characteristics of potential donors, motivation behind donation, as well as clinical findings that preclude eventual donation deserves attention.

Objectives: The objectives of this study were to determine the socio-demographic characteristics of potential living kidney donors, donor motivation, incidental findings on clinical evaluation and some identified factors that prevented eventual kidney donation

Methods: A retrospective review of clinical data of potential living donors who presented for pre-donation evaluation over a period of 20 months (August 2017-March 2019). Age, gender, occupation, relationship to prospective recipient, motivation behind donation, clinical findings and exclusion criteria were collated.

Results: Information for thirty two (32) potential living donors was retrieved. Mean age was $26 \pm 6.5$ years. There were 30 males and 2 females with M:F ratio of 15:2. Majority were unskilled workers (59\%) and unrelated to prospective recipients (68.7\%). Among those related to the patients (31.3\%), there were more second degree relatives (18.8\%) than first degree relatives (12.5\%). The motivations behind donation were fear of losing a loved one to death (81.3\%), altruism (12.5\%) and financial gain (6.2\%). Some incidental findings identified on clinical evaluation included proteinuria (15.6\%), hypertension (12.4\%), bradycardia (6.2\%), abnormal glucose tolerance (3.1\%), cardiomegally $(3.1 \%)$, bacteriuria (3.1\%), renal cysts (3.1\%). Some exclusion criteria findings included immunological incompatibility $(31.3 \%)$, proteinuria (15.6\%), hypertension (12.4\%), financial motivation $(6.2 \%)$ and abnormal glucose tolerance (3.1\%), abnormal cardiac tests (3.1\%).

Conclusion: The potential living donors were mostly young males that were unrelated to the potential recipient. Main motivation behind kidney donation was fear of losing a loved one to death. Incidental findings on laboratory investigations were remarkable while immunologic incompatibility was the leading cause of failure to donate a kidney.

\section{Abstract 97:}

\section{KNOWLEDGE, ATTITUDE AND PERCEPTIONS TOWARDS KIDNEY DONATION AMONG NURSING STUDENTS AT THE COLLEGE OF MEDICINE AND HEALTH SCIENCES, UNIVERSITY OF RWANDA}

Michel Nkuranyabahizi ${ }^{a}, L^{2}$ Lashmi Rajeswaran ${ }^{\mathrm{a}, \mathrm{b}}$, Flavien Ngendahayo ${ }^{\mathrm{a}}$, Emmanuel Gapira ${ }^{\mathrm{a}}$, Geldine Chironda ${ }^{\mathrm{a}, \mathrm{b}}$, Didace Ndahayo $^{\mathrm{a}}$, Theos Marie Pierre Mbambazi ${ }^{\mathrm{a}}$, Baziga Vedaste ${ }^{\mathrm{a}}$ a. University of Rwanda, College of Medicine and Health Sciences, School of Nursing and Midwifery, Kigali, Rwanda; $b$ New York University, Rory Meyers College of Nursing, New York, USA, Human Resources for Health (HRH) Rwanda.

Background: Kidney dysfunction and failure account for increased mortality rates across most population groups globally. Kidney donation and transplantation is a process whereby individuals agree to donate a healthy organ that is then transplanted to the body of a person with either a damaged, failed or dysfunctional organ in order to save their lives as well as improving the quality of life. The current literature does not show the published evidence on the knowledge, attitude and perception towards organ donation in nurses in Rwanda. The aim of this study was to explore knowledge, attitudes and perceptions towards organ donation among nursing students at the University of Rwanda. Methods: A cross-sectional study design using quantitative approach was chosen to meet the objectives of the study. One hundred seventy nine (179) students at the University of Rwanda, College of Medicine and Health Sciences, School of Nursing and Midwifery, Rwamagana campus, participated in the present study. Participants were randomly 
selected. Data were analyzed quantitatively and relationship between knowledge, attitude, perception and socio demographic characteristics variables was reported using chi-square test at the level of significance $<$ or $=$ at 0.05 Results: The results showed low level of knowledge on kidney donation among study participants (51\%). Attitudes were reported to be negative in $63 \%$ of cases and no association was found between knowledge and attitude towards kidney organ donation among study participants. Perception were reported by the big majority of the participants that would prefer to donate their organ to a family member as reported by $68.6 \%$ while $36.9 \%$ of the study participants believe that the greatest important factor to consider when they decide to donate their organ in a case there is a need would be their close relative.

Conclusion and recommendation: The study reported not satisfactory results on knowledge and attitude of study participants. This is a big issue since these future nurses will be expected to support kidney donation in one way or another. Interventions to change the current situation are urgently needed.

\section{Abstract 98:}

\section{FAILURE TO LAUNCH: EXCLUSION FROM LIVING KIDNEY DONATION AT CHARLOTTE MAXEKE JOHANNESBURG HOSPITAL}

Chandni Dayal ${ }^{1}$, Malcolm Davies ${ }^{1}$, Nina Diana ${ }^{1}$, Anthony M. Meyers ${ }^{2}$, Graham Paget ${ }^{1}$

${ }^{1}$ Department of Internal Medicine, Division of Nephrology, Faculty of Health Sciences, University of Witwatersrand, South Africa; ${ }^{2}$ Emeritus Professor of Medicine in the Faculty of Health Sciences, University of Witwatersrand, South Africa.

Background: Living donation offers the potential to significantly expand kidney transplantation with superior quality grafts which are more likely to have higher rates of HLA matching, in turn improving long-term allograft survival. Nevertheless, rates of living donation in many centres are low. We analysed outcomes of evaluation of potential living donors (PLDs) at CMJAH to characterize reasons for exclusion from donation.

Methods: We retrospectively reviewed 1208 PLDs evaluated at our Living Donor Clinic over a 32-year period. Of the total cohort, 298 ultimately donated a kidney, resulting in 910 Failed Living Donors (FLDs). We evaluated donor demographics, donation patterns and reasons for non-donation in the FLD group.

Results: Of 910 FLDs, 522 (57.4\%) were female. The majority were of black African origin (474, 52.1\%) and Caucasian (358, 39.3\%). The greatest number of FLDs were aged 30-39 years (315, 34.6\%). With respect to donor patterns 722 (79.3\%) FLDs were potential related living donors, 180 (19.8\%) were potential directed non-related living donors, only $8(0.9 \%)$ were potential non-directed non-related living donors. Most FLDs (82\%) aged 22-49 years planned to donate sideways to recipients of a similar generational group. Outcome of PLD evaluation varied significantly by race $(p<0.001)$, with only a third of black PLDs being accepted for donation. Black vs. Caucasian PLDs were more likely to fail work-up (77\% vs. 71\%; $p=0.04$ ) and be excluded for medical reasons ( $44 \%$ vs. 35\%; $p<0.001)$. Leading medical exclusions included hypertension, HIV and obesity. 228 (25\%) of the FLDs were medically accepted for donation but did not donate a kidney due to a variety of reasons.

Conclusions: There is a significant exclusion rate for PLDs. Black PLDs are more likely to be excluded than Caucasian counterparts due to significant comorbidity.

\section{Abstract 99:}

\section{LONG TERM OUTCOMES IN ACCEPTED LIVING DONORS AT CHARLOTTE MAXEKE JOHANNESBURG ACADEMIC HOSPITAL}

Chandni Dayal ${ }^{1}$, Malcolm Davies ${ }^{1}$, Nina Diana ${ }^{1}$, Anthony M. Meyers ${ }^{2}$, Graham Paget $^{1}$

${ }^{1}$ Department of Internal Medicine, Division of Nephrology, Faculty of Health Sciences, University of Witwatersrand, South Africa; ${ }^{2}$ Emeritus Professor of Medicine in the Faculty of Health Sciences, University of Witwatersrand, South Africa.

Background: Emerging data suggests the risk of adverse outcomes in kidney donors may have been underestimated. There is a paucity of data regarding outcomes in Accepted Living Donors (ALDs) among demographically diverse populations.

Methods: A retrospective review of 298 ALDs attending the Charlotte Maxeke Johannesburg Academic Hospital Living Donor Clinic over a 32-year period was undertaken. 11 ALDs were excluded due to unavailable pre-donation 
parameters. The following data was collected: pre-donation and post-donation (at first visit, 1 year and most recent visit) blood pressure (BP), albumin-excretion-rate (AER), creatinine and CKD-EPI eGFR. Mortality data was noted.

Results: Median follow-up time was 44 months (IQR 13.8 - 93.5 months). Hypertension was documented in $12.8 \%$ of ALDs at most recent follow up compared to $4.7 \%$ of ALDs pre-donation ( $p=0.06)$. There was a significant increase in AER between pre-donation and post-donation (most recent) visit $(p<0.001)$. There was a significant decline in CKD-EPI eGFR between pre-donation $\left(91.7 \pm 19.1 \mathrm{ml} / \mathrm{min} / 1.73 \mathrm{~m}^{2}\right)$ and most recent visit post donation $\left(72.5 \pm 20 \mathrm{ml} / \mathrm{min} / 1.73 \mathrm{~m}^{2}\right)$ visits $(p<0.001)$. Twenty-seven percent of ALDs had a CKD-EPI eGFR $<60 \mathrm{ml} / \mathrm{min} / 1.73 \mathrm{~m}^{2}$ at most recent visit $(p=0.02)$. No ALDs required institution of renal replacement therapy (RRT). There were 5 documented deaths, 1 in the perioperative period; the remaining 4 were trauma related. Black ethnicity was not associated with increased risk of adverse outcome.

Conclusions: Our data assesses the safety of living kidney donation in the South African population but is limited by high rates of donor follow-up defaulting. Whilst there was a significant increase in the AER over time, the post donation (most recent visit) AER was still within normal limits. There is concern that $25 \%$ of the ALDs developed an eGFR $<60 \mathrm{ml} / \mathrm{min} / 1.73 \mathrm{~m}^{2}$, however none have required RRT at last follow-up.

\section{Abstract 100:}

\section{THE INFLUENCE OF DIALYSIS MODALITY ON POST TRANSPLANT OUTCOMES}

Dr R Boosi ${ }^{1}$, Dr M Davies ${ }^{1}$, Dr F Khan ${ }^{1}$

${ }^{1}$ Nephrology Unit, Dept of Internal Medicine, University of the Witwatersrand.

Introduction: Renal transplantation is the therapy of choice for end stage kidney disease, offering mortality risk reduction and improved morbidity over dialytic therapies. Limited data is available evaluating the effect of preengraftment dialysis modality on transplant outcomes.

Methods: A retrospective review was conducted on all adult patients undergoing renal transplantation at Charlotte Maxeke Johannesburg Academic Hospital for the period 01/01/2006 - 31/12/2011 (n=103). Transplant outcomes were assessed by dialysis modality. [?] [? modelling was used to assess effect on graft outcomes. A $p<0.05$ was deemed statistically significant.

Results: Antecedent dialytic modality was as follows: 55 patients (53.4\%) received hemodialysis (HD), 35 (34\%) received peritoneal dialysis (PD), and $13(12.6 \%)$ received a combination of both (HD+PD, defined as either modality for $>3$ months). Acute rejection (AR) was documented in $43.7 \%$ of patients; $54.3 \%$ of PD patients developed AR compared to $38.2 \%$ of HD patients and $38.5 \%$ of HD+PD patients $(p=0,29)$. No significant difference in the number of episodes of AR was detected between modality groups $(p=0.44)$. Chronic rejection $(C R)$ developed in $22.3 \%$ of patients overall; $21.8 \%$ of HD patients, $25.8 \%$ of PD and $15.9 \%$ of HD+PD patients $(p=0,74)$. PD was associated with an increased risk of developing any rejection ( $\mathrm{HR}=2,4,95 \% \mathrm{Cl} 0,9-6,4, \mathrm{p}=0,02)$. Whereas dialysis modality did not affect graft survival $(p=0.24)$, AR was associated with future graft loss $(p<0.001)$.

Conclusions: Antecedent PD is associated with an increased risk of graft rejection. Although AR is associated with graft loss, antecedent dialysis modality does not directly predict graft survival, likely reflecting the multifactorial nature of cumulative allograft injury.

\section{MISCELLANEOUS}

\section{Abstract 101:}

\section{HIGH ARTERIAL PRESSURE IN YOUNG PARAKOU SECONDARY TEACHERS IN 2019: PREVALENCE AND ASSOCIATED FACTORS}

Séraphin AHOUI ${ }^{1}$, Comlan A DOVONOU ${ }^{1}$, Bruno L AGBOTON ${ }^{2}$, Hermann BIAO ${ }^{1}$, Romaric SEGO-SOUNON ${ }^{3}$, Madinatoulaye $\mathrm{CHABI}^{3}$ Jacques VIGAN ${ }^{2}$, Léopold $\mathrm{H} \mathrm{CODJO}^{1}$

${ }^{1}$ Faculty of Medicine, University of Parakou; ${ }^{2}$ Faculty of Health Sciences, University of Abomey -Calavi; ${ }^{3}$ Training Institute in Nursing and Obstetric Care.

Background: High arterial pressure remains one of the risk factors for cardiovascular disease. Objective: To study the prevalence and factors associated with high arterial pressure among high school teachers at the age of 45 years in the city of Parakou in 2019. 
Methods: This was a descriptive and analytical cross-sectional study. Data collection took place from February $1^{\text {st }}$ to February $28^{\text {th }}, 2019$ and was conducted on teachers at three public colleges in Parakou over the age of 45 years. The choices of colleges and teachers were random. The minimum sample size was 303 teachers. High arterial pressure (HAP), a dependent variable, was selected at $\geq 140 / 90 \mathrm{mmHg}$ after three sit-ups and one after 15 minutes of rest. Socio-demographic, clinical and lifestyle variables were studied. The data was entered using Epi Data 3.1 and analyzed using Epi info7 with a significance level of $5 \%$.

Results: A total of 313 teachers participated. The mean age was 35.30 years \pm 5.64 years and the sex ratio was 6.45 . The prevalence of blood pressure was $16.60 \%$. HAP was significantly associated with obesity, smoking, diet, sedentary lifestyle, home-work distance, gestational / parity in women and stress

Conclusion: The prevalence of HBP among young teachers is important. Better prevention of its contributing risk factors to reduce the morbidity and mortality of cardiovascular diseases. Key words: High blood pressure, young teachers, Parakou Benin

\title{
Abstract 102:
}

RENAL ULTRASOUND BIOMETRY IN APPARENTLY HEALTHY BLACK SUBJECTS IN OUAGADOUGOU (BURKINA FASO)

\author{
J. Y. BONZI ${ }^{1}$, G. CAPO-CHICHI ${ }^{1}$, O. DIALLO ${ }^{2}$, A. OUATTARA ${ }^{2}$, A. LENGANI ${ }^{1}$, G. SANOU ${ }^{1}$, I. NITIEMA ${ }^{1}$, H. TRAORE ${ }^{1}$, G. \\ COULIBALY ${ }^{1}$ \\ 1-nephrology-hemodialysis departement of yalgado OUEDRAOGO University Hospital; 2-Radiology departement of \\ yalgado OUEDRAOGO University Hospital.
}

Objective: The average renal ultrasound biometric values $(120 \times 60 \times 30 \mathrm{~mm})$ used in current practice come from Caucasian population series. In this study we evaluate these values in apparently healthy black subjects in Ouagadougou

Methods: Ultrasound measurements (height, width and thickness) of the kidneys were performed by two radiologists in 100 volunteers (69 male and 31 female) aged 18 to 30 years and presumed healthy with a multi-parameter urine strip and normal plasma creatinine. Subjects' informed consent was obtained before their inclusion. The ultrasound devices were TOSHIBA ${ }^{\mathrm{TM}}$ and ELECTRIC MOTOR ${ }^{\mathrm{TM}}$.

Results: Subjects were on average $24 \pm 6.5$ years of age. The average weight and body mass index were $68 \pm 5 \mathrm{~kg}$ and $22.6 \mathrm{~kg} / \mathrm{m}^{2}$ respectively. The ultrasound dimensions of the right kidney averaged $94.5 \pm 14.5 \mathrm{~mm}$ (height), $38.7 \pm 10.7$ $\mathrm{mm}$ (width) and $36.3 \pm 10.3 \mathrm{~mm}$ (thickness). The dimensions of the left kidney were $96.7 \pm 16.7 \mathrm{~mm}$ (height), $42.7 \pm 16.7$ $\mathrm{mm}$ (width) and $37 \pm 11 \mathrm{~mm}$ (thickness). The dimensions followed a normal distribution in the study population with a $95 \%$ probability of having a renal height between $79-110 \mathrm{~mm}$ on the right and $81.5-112 \mathrm{~mm}$. A size $\geq 170 \mathrm{~cm}$ and a BMI $>25 \mathrm{~kg} / \mathrm{m}^{2}$ were significantly associated with larger than average kidney sizes.

Conclusion: This study indicates that there may be a difference in kidney size between Caucasian and Burkina Faso populations.

\section{Abstract 103: \\ PRESCRIPTION OF PHARMACOTHERAPY AND BLOOD PRESSURE CONTROL AMONG HYPERTENSIVE OUTPATIENTS IN TWO HOSPITALS OF SEMI-URBAN AREA IN CAMEROON: A CROSS-SECTIONAL STUDY}

Anastase Dzudie, Messaline Fomo Fodom, Denis George Teuwafeu, Clovis Nkoke, Azabji Kenfack, Divine Tim Bonghaseh, Domin Ekaney, Amy Tantchou, Cabral Tantchou, Laurent Serges Etoundi Ngoa, Andre Pascal Kengne, Pierre Simeon Choukem

Background: Several international guidelines are available on drugs treatment for hypertension, but the control of hypertension remains suboptimal and very poor in Sub-Saharan Africa (SSA). We investigated the commonly prescribed antihypertensive drugs and their association with blood pressure (BP) control in adult Africans.

Methods: We consecutively treated hypertensive outpatients attending the Buea and Limbe Regional hospitals (Southwest region of Cameroon). Controlled BP was defined as BP $<140 / 90 \mathrm{mmHg}$ in hypertensive patients less than 60 years old, diabetics or patients with chronic kidney disease or a $\mathrm{BP}<150 / 90 \mathrm{mmHg}$ in non-diabetic hypertensive patients greater than 60 years old (JNC8).

Results: Of 408 participants enrolled (mean age 61.1 years), 67\% were female. The mean duration of hypertension was $8.5 y e a r s$ and the median duration of current treatment was 22 weeks. Commonly prescribed antihypertensives were calcium channel blockers (CCB, 35.1\%), thiazide/thiazide-like diuretics (TD/TLD,26.1\%) and angiotensin 
converting enzyme inhibitors (ACEI,19.5\%). The median monthly cost of antihypertensive was 10279.6 CFA (US\$ 172). $70 \%$ of participants were receiving at least 2 drugs, with ACEI+TD/TLD, CCB+TD/TLD and ACEI+CCB+TD/TLD being the most frequent. The rate of $\mathrm{BP}$ control was $52 \%$ overall, and $60 \%$ in participants on monotherapy.

Conclusion: $C C B$ were the most prescribed single antihypertensive drugs in this setting while ACEI+TD/TLD was the most common combination. About five to six in 10 patients were at target BP control level Improving availability and affordability of these medications may improve hypertension control and management.

\section{Abstract 104:}

\section{HYPONATRAEMIA AND MORTALITY IN MEDICAL ADMISSIONS IN GHANA - A SINGLE CENTRE STUDY}

Elliot Koranteng Tannor ${ }^{1}$, Emmanuel Owusu Akumiah² Betty Norman ${ }^{3}$

${ }^{1}$ Renal Unit, Department of Medicine, Komfo Anokye Teaching Hospital, Kumasi, Ghana; ${ }^{2}$ Research and development Unit, Komfo Anokye Teaching Hospital, Kumasi, Ghana; ${ }^{3}$ Department of Medicine, School of Medical Science, Kwame Nkrumah University of Science and Technology.

Introduction: Hyponatremia is the most common electrolyte abnormality in hospital admissions. It is associated with poor prognosis in most medical conditions. There is paucity of data on hyponatraemia in Ghana. We set out to describe the prevalence of hyponatraemia, its associations and determine the outcome of patients admitted with hyponatremia in terms of in-hospital mortality and length of hospital stay.

Methods: We conducted a retrospective study of all medical admissions from $1^{\text {st }}$ October 2017 to $30^{\text {th }}$ April 2018 on the medical ward at the Komfo Anokye Teaching Hospital (KATH). Means \pm SD and medians with interquartile range were used to describe the data where appropriate. Chi square and Fisher exact test were used to test categorical variables where appropriate. ANOVA and Kruskal-Wallis and were used to test differences with severity of hyponatremia and a $p$ value of $<0.05$ was considered statistically significant.

Results: Within the study period, 406 patients with hyponatraemia were identified in 1477 medical admissions. The prevalence of hyponatraemia was $27.6 \%$. The mean age was $51.5 \pm 19.0$ years. There were $217(53.5 \%)$ males. The mean serum sodium was $128.7 \pm 6.5 \mathrm{mmol} / \mathrm{L}$. Two hundred and forty $(59.1 \%)$ had mild hyponatremia, $106(26.1 \%)$ had moderate hyponatraemia and $60(14.8 \%)$ had severe hyponatremia. The most common medical conditions associated with hyponatraemia were infections $105(25.9 \%)$, chronic liver disease 69(17.0\%), hyperglycaemia 68(16.8\%), chronic kidney disease $66(16.3 \%)$ and 33(8.1\%) with chronic heart failure. Mortality on admission was $129(31.8 \%)$. Mortality rate varied with severity of hyponatremia mild 50(20.8\%), moderate $48(45.3 \%)$ and severe $31(51.7 \%) p<0.001$. Median length of hospital stay was 7 days (IQR 4-10) and did not vary significantly with severity of hyponatraemia $(p=0.64)$. Mortality was predicted by serum sodium concentration $(p=0.007)$ and state of unconsciousness at presentation $(p<0.001)$.

Conclusion: Hyponatraemia is common in medical admissions and associated with increased mortality. Mortality increased with severity of hyponatraemia and state of unconsciousness.

\section{Abstract 105:}

\section{BRIDGING THE GAP BETWEEN ACADEMIA AND PREVENTION OF KIDNEY DISEASES IN GHANA - THE ROLE OF HEALTH EDUCATION}

Elliot Koranteng Tannor, ${ }^{1}$ Emmanuel Akumiah' ${ }^{2}$, Betty R Norman ${ }^{3}$

${ }^{1}$ Renal Unit, Department of Medicine, Komfo Anokye Teaching Hospital, Kumasi, Ghana; ${ }^{2}$ Reseaarch and Development Unit, Komfo Anokye Teaching Hospital, Kumasi; ${ }^{3}$ Department of Medicine, Komfo Anokye Teaching Hospital, Kumasi, Ghana.

Introduction: Medical education in Ghana involves long years of studies to be a specialist and Nephrologist. After training, most astute clinicians enter into academia and are located in tertiary hospital far from the reach of the ordinary Ghanaian. Most patients with kidney disease present late with complications requiring renal replacement therapy, which most cannot afford. Awareness creation and preventive care is likely to decrease prevalence and mortality from kidney diseases. We set out to educate the populace and train health care staff on the management of kidney disease in Ghana.

Methods: We rolled out education in kidney diseases and its causes in the Ashanti region in Ghana. Targets for education included primary care doctors, nurses and health care providers on how to screen, identify, manage and appropriately refer patients with kidney disease. We also educated the general public on prevention of kidney 
diseases via social media portals, electronic media, and radio and television programs. Organized groups such as churches were also targeted in 2 years.

Results: Health education have reached over 15 radio stations on several occasions, 4 television stations and 20 local churches reaching millions of Ghanaians within 2 years. We have also organized free screening exercises at 15 different locations some with support from a local non-governmental organization. We have also written 5 articles on health promotion on electronic media. There has been the formation of renal management teams in 5 district hospitals in the Ashanti region. Five continuous development programs for doctors and health staff have been held in the Ashanti region.

Conclusion: Primary health professionals should be actively involved in preventive nephrology to promote health and prevent kidney disease in Ghana.

\section{Abstract 106:}

\section{INVESTIGATING THE POSSIBILITY OF A PERIOD EFFECT OF MEMBRANE FILTRATION DEVICE IMPLEMENTATION ON REDUCING DIARRHEA INCIDENT RATES IN RURAL COMMUNITIES IN GHANA}

Joseph Marfo Boaheng ${ }^{1}$, Jochen G. Raimann ${ }^{1}$, Philip Narh ${ }^{2}$, Seth Johnson ${ }^{1}$, Linda Donald ${ }^{1}$, Hongbin Zhang ${ }^{3}$, Friedrich Port $^{1}$, Nathan W. Levin ${ }^{1}$.

1. Easy Water for Everyone, New York, NY, United States; 2. Ghana Health Service, Ada, Ghana; 3. Graduate School of Public Health and Health Policy, New York, NY, United States.

Introduction: The Sustainable Development Goal (SDG) 6-target 3 of the United Nations emphasizes accessibility to drinkable water to improve public health worldwide. Contaminated water is a risk factor for infectious diarrhea and often leads to acute kidney injury and death particularly in the young and elderly.

Objective: We investigated the possibility of a period effect on the treatment effect of membrane filtration device implementation (NUF500; NUFiltration, Israel) for water purification using recycled hemodialyzers on the incidence rates of diarrhea in rural Ghana.

Methods: Data was collected within two separate time periods between 03/2018 to 02/2019 in 14 communities. Differences between before and after implementation of the devices was tested using Welch's test for unequal variances. Data are presented as mean (sd) unless stated otherwise. A p-value less than $5 \%$ was considered significant. Results: We studied data on the occurrence of diarrhea among 2 groups stratified by villages, comprising 2605 villagers and found average monthly rates prior to implementation of $0.08(0.04)$ for group 1 and $0.13(0.14)$ diarrhea events per subject-months for group 2. For the post periods, the average incidence rates were $0.03(0.02)$ and 0.03 (0.13) per subject-months, respectively. We found statistically significant differences between the incidence rates pre and post device implementation for both groups [group $10.05(0.02)$ and group $2(0.10(0.01)$ ]. Effects did not significantly differ between both groups, thus confirming the reduced incidence rates in diarrhea after the implementation of the filtration device without evidence of a period effect in this analyses.

Conclusion: Our data suggest a significantly lower diarrhea incidence rates following the implementation of the membrane filtration device in rural communities in Ghana. This effects were independent from a period effect despite the proximity of villages and the concomitantly conducted handwashing and hygiene initiative launched in all villages in the same region. In concert with previously reported results our data support public health efforts such as those of the United Nations, the WHO and our very own, Easy Water for Everyone, that aim to allow access to clean water for everyone. The effect of education programs on the importance of handwashing and hygiene will be subject of future investigations.

\section{Abstract 107:}

\section{INCIDENCE OF CONTRAST INDUCED NEPHROPATHY}

Chepkwony Chebet

Moi University School of Medicine.

Background: Contrast Induced Nephropathy (CIN) is defined as acute kidney injury post intravenous contrast media (CM). In CIN, creatinine levels peak at 3-5 days and return to normal in 14 days. One third of the patients with CIN develop residual Chronic Kidney Disease. In this study, CIN was defined as a rise in creatinine level of $\geq 44 \mathrm{umol} / \mathrm{I}$ from baseline. 
Objective: To determine the prevalence and risk factors for developing CIN among patients undergoing IV contrast based studies in Moi Teaching and Referral Hospital (MTRH), Kenya.

Methods: A cross sectional study was conducted at Radiology department and MTRH wards on patients without kidney disease at baseline. Omnipaque was used and the dose was $1-3 \mathrm{ml}$ per kg body weight.

Patients were consecutively recruited and followed up for 14 days. Dehydrated patients were rehydrated with IV fluids before CM administration. On day 3, kidney functions were assessed for CIN development and recovery from it on day 14. Age, gender, physical examination findings, hematocrit, serum albumin, creatinine \& urea), urine analysis and fasting blood sugar (FBS) were obtained and recorded. Prevalence was calculated as the proportion of patients who developed $\mathrm{CIN}$ on day 3pos contrast media administration. Descriptive statistics were summarized in tables and graphs and correlation done using Pearson's Chi Square.

Results: 120 patients were recruited. Median age was 48.5 years I.Q.R (33.5-60.5). Proportion of CIN using an absolute increase of creatinine from baseline was 34\%, (95\% Cl-25.53-42.47). Dehydration was at 35\%, $12 \%$ had hypertension, $18 \%$ had anemia and $12 \%$ had diabetes and $16 \%$ were pre-diabetics. $97 \%$ were inpatients and $3 \%$ were outpatients. Hypertension was a predictor for CIN, 95\% Cl, OR 16.42(1.51-179) p=0.0001. Anemia also predicted CIN, 95\% Cl OR 7.06(1.70-29.28) $p=0.007$.

Conclusion: Prevalence of CIN in MTRH stood at 34\%. Hypertension and being anemic were predictors for CIN. High hematocrit and higher albumin were protective of CIN.

\title{
Abstract 108:
}

\section{THE ASSOCIATION BETWEEN BLOOD PRESSURE CONTROL AND ADHERENCE TO ANTIHYPERTENSIVES AND LIFESTYLE THERAPY AMONG HYPERTENSIVES IN A SEMI-RURAL NIGERIAN COMMUNITY}

\author{
C. Ndulue ${ }^{1}$, A. Anyanor ${ }^{1}$, B. Ozuemba ${ }^{1}$, A. Osakwe ${ }^{1}$, F. Oguejiofor ${ }^{1}$, H. Eze ${ }^{1}$, N. Jisieke-Onuigbo ${ }^{1}$, C. Odenigbo $^{1}$, I. Ulasi $^{2}$. \\ ${ }^{1}$ Nnamdi Azikiwe University Teaching Hospital, Medicine, Nnewi, Nigeria; ${ }^{2}$ University of Nigeria Teaching Hospital, \\ Medicine, Enugu, Nigeria.
}

Introduction: Hypertension is a public health challenge among Nigerians and optimal blood pressure (Bp) control is of paramount importance. Several studies on the relationship between antihypertensive compliance only and Bp control have been described among Nigerians. The objective of the present study was to examine the association between Bp control and adherence to pharmacologic and non-pharmacologic therapies among previously diagnosed hypertensives in a semi-rural Nigerian community.

Methods: Using a cross-sectional design, interviewer-administered questionnaires were used to obtain information on demographics, compliance to medications and lifestyle modification (low salt diet, exercise, weight loss, smoking cessation, and reduced alcohol intake). Blood pressure, BMI and waist circumference were measured in a standardized manner. The primary outcome was blood pressure control, which was defined as measured Bp $\leq 140 / 90$ $\mathrm{mmHg}$. Univariate logistic regression was used to determine the factors that influence Bp control.

Results: $48 \%$ of 184 adults examined were known hypertensives and $71 \%$ of hypertensives were female. $34 \%$ were adherent to medication while $80 \%$ complied with at least one of the lifestyle measures. Only $14 \%$ of hypertensives had good bp control.

Conclusion: Bp control among hypertensives in this community study is low.

\section{Abstract 109:}

\section{AGREEMENT BETWEEN POINT-OF-CARE BLOOD GAS ANALYZER AND LABORATORY AUTOANALYZER MEASUREMENTS IN PATIENTS WITH HYPERKALAEMIA}

MY Chothia and P Kassum

Division of Nephrology, Department of Medicine, Faculty of Medicine and Health Sciences, Stellenbosch University and Tygerberg Hospital.

Background: Hyperkalaemia is a common electrolyte disorder that may cause life-threatening cardiac arrythmias. Objective: To determine the level of agreement between point-of-care blood gas analyzer (POC-BGA) and laboratory auto-analyzer potassium concentrations in patients with hyperkalaemia.

Methods: This was a prospective, cross-sectional study of all consecutive adult patients referred to the Renal Unit with a serum potassium concentration $>5.5 \mathrm{mmol} / \mathrm{L}$. Two simultaneous blood samples were taken using a standardized phlebotomy technique. Patients with hyperleukocytosis and thrombocytosis were excluded. 
Results: A total of 59 paired blood samples were included in the final statistical analysis. The median laboratory autoanalyzer potassium concentration was $6.1 \mathrm{mmol} / \mathrm{L}$ as compared to the POC-BGA potassium concentration of 5.7 $\mathrm{mmol} / \mathrm{L}(\mathrm{p}<0.01)$ with a mean bias of $-0.43 \mathrm{mmol} / \mathrm{L}$. There was a very strong correlation between the two methods ( $r$ $=0.876$ ); however, agreement was poor with $52.5 \%$ of values outside of the total allowable error. $96.6 \%$ of values were within the reference change value.

Conclusion: Despite the poor agreement between POC-BGA and laboratory auto-analyzer potassium concentrations, a very strong correlation exists. Therefore, POC-BGA potassium concentrations can be used as a surrogate measurement for laboratory auto-analyzer measurements in patients with hyperkalaemia after adjustment for this systematic negative bias.

\section{Abstract 110:}

\section{A GLOMERULAR FILTRATION RATE PREDICTION EQUATION DEVELOPED IN AFRICAN PATIENTS IN DURBAN, SOUTH AFRICA}

Madala ND ${ }^{1,2 *}$, Oluyede $\mathrm{M}^{3}$, Tapamo $\mathrm{JR}^{3}$, Naicker $\mathrm{S}^{4}$, Assounga $\mathrm{AGH}^{1}$

${ }^{1}$ Department of Nephrology, Nelson R Mandela School of Clinical Medicine, University of KwaZulu-Natal, Durban, South Africa; ${ }^{2}$ Department of Internal Medicine, Sefako Makgatho Health Sciences University, Ga-Rankuwa, Pretoria, South Africa; ${ }^{3}$ School of Electrical, Electronic and Computer Engineering, University of KwaZulu-Natal, Durban, South Africa; ${ }^{4}$ School of Clinical Medicine, Faculty of Health Sciences, University of Witwatersrand, Johannesburg, South Africa.

Background: Existing serum creatinine-based estimated glomerular filtration rate (eGFR) equations are inaccurate in sub-Saharan Africans. African-American derived black ethnicity coefficients in currently-used eGFR equations result in GFR overestimation. The objective of this study was to develop a GFR estimating equation in Africans by modifying the Modification of Diet in Renal Disease (MDRD) equation to improve GFR prediction.

Methods: We enrolled 76 adult black African chronic kidney disease (CKD) patients. Technicium-99diethylenetriaminepentaacetic acid ( ${ }^{99 \mathrm{~m}}$ Tc-DTPA) clearance was used as the gold standard GFR method. GFR prediction equations were developed from multivariable linear regression analysis models using the whole dataset for training and testing. Variables used in the 6-variable MDRD equation were modelled, namely age, gender, serum creatinine, urea, albumin, except ethnicity. Bias, precision and accuracy were calculated to evaluate concordance between ${ }^{99 m}$ Tc-DTPA-GFR and eGFR.

Results: Mean ${ }^{99 \mathrm{~m} T c-D T P A-G F R}$ was $51.3 \pm 22.6 \mathrm{ml} / \mathrm{min} / 1.73 \mathrm{~m}^{2}$ and $89.6 \% \mathrm{had}$ CKD. The modified MDRD equation for Africans showed the best GFR prediction, with the lowest bias $(95 \% \mathrm{Cl})$ of $-0.72(-5.17,3.73) \mathrm{ml} / \mathrm{min} / 1.73 \mathrm{~m}^{2}$ and root mean square error, $13.4 \mathrm{ml} / \mathrm{min} / 1.73 \mathrm{~m}^{2}$ as well as highest accuracy within $30 \%$ of measured GFR of $75 \%$. MDRD-eGFR performance was $6.21(2.75,9.67) \mathrm{ml} / \mathrm{min} / 1.73 \mathrm{~m}^{2}, 18.1 \mathrm{ml} / \mathrm{min} / 1.73 \mathrm{~m}^{2}$ and $51.3 \%$ and by Chronic Kidney Disease Epidemiology Collaboration (CKD-EPI) eGFR, $3.56(0.27,6.82) \mathrm{ml} / \mathrm{min} / 1.73 \mathrm{~m}^{2}, 17.1 \mathrm{ml} / \mathrm{min} / 1.73 \mathrm{~m}^{2}$ and $63.2 \%$ for bias, RMSE and accuracy, respectively.

Conclusion: The GFR prediction was improved by using an equation developed in Africans. An African modified equation may overcome the inaccuracy experienced with MDRD-eGFR and CKD-EPI eGFR. 